\title{
SAR IMAGING OF MOVING TARGETS BY SUBAPERTURE BASED LOW-RANK AND SPARSE DECOMPOSITION
}

by

Mubashar Yasin

Submitted to the Graduate School of Sabancı University in partial fulfillment of the requirements for the degree of Master of Science

Sabancı University

August 2017 
SAR IMAGING OF MOVING TARGETS BY SUBAPERTURE BASED LOW-RANK AND SPARSE DECOMPOSITION

APPROVED BY:

Assoc. Prof. Dr. Müjdat Çetin

(Thesis Supervisor)

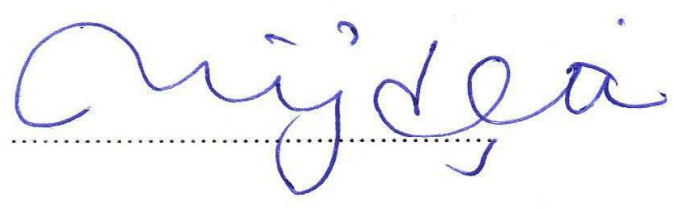

Assist. Prof. Dr. Kamer Kaya

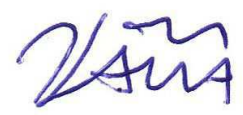

Assist. Prof. Dr. N. Özben Önhon

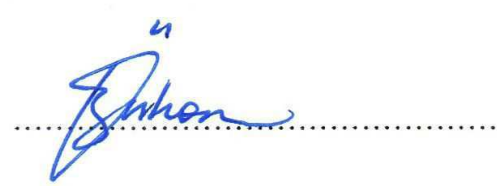

DATE OF APPROVAL: $\quad 1^{\text {st }}$ August, 2017 
(C) Mubashar Yasin 2017

All Rights Reserved 
to my parents 


\section{Acknowledgments}

I feel honored and happy to write my research thesis for the masters degree at Sabancı University. I like to thank Allah Almighty for giving me the knowledge, courage, and ability for this accomplishment.

Foremost, I would like to say very special thanks to my research advisor Dr. Müjdat Çetin for his persistent support, guidance, immense patience and kindliness in the course of this research. It was a great honor to work with him and I learned a lot from him during my graduate studies at Sabancı University. Also, I would like to express my gratitude for Dr. Ahmed Shaharyar Khwaja. He was always very generous and supportive. His encouragement and guidance furthered my motivation for this research.

I am very thankful to Dr. Kamer Kaya for his kindliness and being a jury member for my thesis. Furthermore, it was a great learning from him about parallel processing and algorithms. I am also very grateful to Dr. N. Özben Önhon for being the external jury member for my thesis. Her PhD work was a great source of inspiration for my research.

I also like to appreciate all VPA lab fellows and SPIS group members for useful interaction, friendship and support. It was an enjoyable experience to work in this research environment.

I am thankful to Professor Erik Lintz Christensen, Associate Professor Jorgen Dall and the Technical University of Denmark (DTU) for providing the EMISAR data for this research.

Finally, I greatly appreciate my family for their love, consistent support, and best wishes for my success. 


\title{
SAR IMAGING OF MOVING TARGETS BY SUBAPERTURE BASED LOW-RANK AND SPARSE DECOMPOSITION
}

\author{
Mubashar Yasin \\ EE, M.Sc. Thesis, 2017 \\ Thesis Supervisor: Müjdat Çetin
}

\begin{abstract}
Keywords: Synthetic aperture radar (SAR), SAR imaging, moving targets, low-rank and sparse decomposition, subaperture processing
\end{abstract}

\begin{abstract}
Synthetic aperture radar (SAR) has gained significance as an indispensable instrument of remote sensing and airborne surveillance. Its applications extend to 3D terrain mapping, oil spill detection, crop yield estimation and disaster evaluation. SAR utilizes platform motion to synthesize a large antenna thus rendering a very fine spatial resolution. Nevertheless, imaging of moving targets with SAR is a challenging problem. In this thesis, we propose a moving target imaging approach for SAR which exploits the low-rank and sparse decomposition (LRSD) of the subaperture data. As a first step, multiple subapertures are constructed from the raw data using frequency domain filtering. In contrast to the stationary points, moving targets in the SAR scene shift their position in the various subapertures. This enables a successful low-rank and sparse decomposition of the subaperture data where the sparse component captures the moving targets' phase histories and reflectivity profiles. On the other hand, the low-rank component consists of the static background due to fewer spatial variations in multiple subapertures. This framework allows the reconstruction of full-resolution sparse and low-rank images by combining the spectral information of the decomposed subapertures. Furthermore, it enhances the applicability of sparsity-driven moving target imaging frameworks to very low signal to clutter ratio (SCR) scenarios by offering
\end{abstract}


a considerable SCR performance improvement. We manifest the effectiveness of our approach through experiments with synthetic as well as real SAR data. Our real SAR experiments were based on MiniSAR and EMISAR data. 


\title{
SAR IMAGING OF MOVING TARGETS BY SUBAPERTURE BASED \\ LOW-RANK AND SPARSE DECOMPOSITION \\ ALTAÇIKLIK TABANLI DÜŞÜK SIRALI VE SEYREK AYRIŞIM İLE \\ HAREKETLİ HEDEFLERIN SAR GÖRÜNTÜLEMESI
}

\author{
Mubashar Yasin \\ EE, Yüksek Lisans Tezi, 2017 \\ Tez Danısmanı: Müjdat Çetin
}

Anahtar Kelimeler: Sentetik açıklıklı radar (SAR), SAR görüntüleme, hareketli hedefler, düşük sıralı ve seyrek ayrışım, altaçıklık işleme

\section{Özet}

Sentetik açıklıklı radar (SAR), uzaktan algılama ve havadan gözetim için vazgeçilmez bir araç olarak önem kazandı. SAR uygulamaları, 3B arazi haritalama, petrol sızıntısı belirleme, tarımsal üretim kestirme ve afet ölçümü gibi farklı alanlara uzanır. SAR, büyük bir anten etkisi oluşturmak ve böylelikle çok daha iyi bir uzamsal çözünürlük sunmak için gözlem platformunun hareketini kullanır. Yine de, hareketli hedeflerin SAR ile görüntülenmesi zorlu bir sorundur. Bu tezde, altaçıklık verisinin düşük kerteli ve seyrek ayrışımını (LRSD) kullanan bir SAR hareketli hedef görüntüleme yaklaşımı öneriyoruz. İlk adım olarak, frekans bölgesi süzgeçlemesi kullanılarak, işlenmemiş veriden çoklu altaçıklıklar oluşturulmuştur. Sabit noktaların aksine, SAR görüntüsündeki hareketli hedefler farklı altaçıklıklarda konum değiştirirler. Bu, altaçıklık verisinin başarılı biçimde düşük kerteli ve seyrek ayrışımını sağlar. Burada, seyrek bileşen, hareketli hedeflerin faz geçmişini ve yansıtırlık profilini yakalar. Diğer yandan, düşük kerteli bileşen ise, çoklu altaçıklıklardaki daha az olan uzamsal değişikliklerden dolayı sabit arkaplandan oluşur. Bu çerçeve, ayrıştırılmış altaçıklıkların görüngesel bilgilerini 
birleştirerek, seyrek ve düşük kerteli görüntülerin tam çözünürlüklü geriçatılmasına izin verir. Buna ek olarak, işaret gürültü oranında (SCR) dikkate değer bir verim artışı sağlayarak, seyreklik güdümlü hareketli hedef görüntüleme yapılarının çok düşük SCR durumlarında uygulanabilirliğini geliştirir. Yaklaşımımızın etkinliğini, sentetik ve gerçek SAR verileriyle yaptığımız deneylerle gösteriyoruz. Deneylerde kullandığımız gerçek SAR verileri, MiniSAR ve EMISAR verileridir. 


\section{Table of Contents}

Acknowledgments $\quad$ V

$\begin{array}{lll}\text { Abstract } & \text { vi }\end{array}$

Özet viii

1 Introduction 1

1.1 Problem Definition and Motivation . . . . . . . . . . . . . . 1

1.2 Contributions made by this Thesis . . . . . . . . . . . . . . . . . 4

1.3 Organization of the Thesis . . . . . . . . . . . . . . . 5

2 Background 6

2.1 SAR Basics . . . . . . . . . . . . . . . . . . . . . . . . . . . . . . . . .

2.1.1 SAR Imaging Modes . . . . . . . . . . . . . . . . . . . 9 9

2.1.2 Imaging Model for the Spotlight Mode . . . . . . . . . . . . . . 9

2.2 Moving Target Imaging with SAR . . . . . . . . . . . . . . . . . 11

2.2.1 Frequency based Moving Target Detection . . . . . . . . . . . . 12

2.2.2 Phase based Moving Target Detection . . . . . . . . . . . . . . 12

2.3 Regularization based Imaging . . . . . . . . . . . . . . . . . . . . 13

2.3.1 Least-squares Solution and Regularization . . . . . . . . . . . 13

2.3.2 Quadratic Regularization . . . . . . . . . . . . . . . . 14

2.3.3 Non-quadratic Regularization ................ 14

2.4 Feature-enhanced SAR Image Reconstruction . . . . . . . . . . . . . . 15

2.4.1 Point-enhanced Reconstruction . . . . . . . . . . . . . 16

2.4.2 Region-enhanced Reconstruction . . . . . . . . . . . . . 16

2.5 Sparsity-driven Moving Target Imaging . . . . . . . . . . . . . . . . . . . . . 16

2.6 Low-rank and Sparse Decomposition (LRSD) . . . . . . . . . . . . . . . 19

2.7 Subaperture Processing . . . . . . . . . . . . . . . . . . . . . 20

2.7.1 Subaperture Imaging of Moving Targets . . . . . . . . . . . . . 20

3 Proposed Method for Moving Target Imaging 24

3.1 Separation of Static and Dynamic Components . . . . . . . . . . . . . 24 
3.2 Subapertures and the Data Matrix for LRSD . . . . . . . . . . . . 26

3.3 LRSD based on Subaperture Data (SLRSD) . . . . . . . . . . . . 27

3.4 Solving the Lagrangian Form of SLRSD . . . . . . . . . . . . . . 28

3.4.1 Update of the Sparse Component $S \ldots \ldots \ldots \ldots$

3.4.2 Update of the Background (Low-rank) Component $B$. . . . . 30

3.4.3 Update of the Composite Matrix $W \ldots \ldots \ldots$. . . . . 30

3.4.4 Update of the Phase Matrix $\Theta \ldots \ldots \ldots \ldots$. . . . . . 30

3.4.5 Update of the Lagrange Multiplier $\Gamma$ and $\beta \ldots \ldots \ldots \ldots . \ldots 31$

3.5 Full-resolution Reconstruction after LRSD . . . . . . . . . . . . . . . . 31

4 Experimental Results $\quad 34$

4.1 Experimental Results with Synthetic Targets . . . . . . . . . . . . . 34

4.1.1 Synthetic Scene Experiments . . . . . . . . . . . . . . 34

4.1.2 Experiments with Real SAR Background . . . . . . . . . 36

4.2 Numerical Simulations for Performance Evaluation . . . . . . . . . . 39

4.2 .1 SCR Performance Analysis . . . . . . . . . . . . . . . . . . . . 39

4.2 .2 Target Velocity Variations . . . . . . . . . . . . . . . . 40

4.2 .3 Varying Number of Subapertures . . . . . . . . . . . . . . 42

4.3 Processing Time and Memory Usage . . . . . . . . . . . . . . . . 45

5 Experiments with EMISAR Data 46

5.1 About EMISAR . . . . . . . . . . . . . . . . . . . . 46

5.2 Moving Target Scene 1 (region-1) $\ldots \ldots \ldots \ldots$

5.3 Moving Target Scene 2 (region-2) . . . . . . . . . . . . . . . . . 54

5.4 Moving Target Scene 3 (region-2) . . . . . . . . . . . . . . . . . 59

5.5 Discussion . . . . . . . . . . . . . . . . . . . . . . . 59

6 Conclusions and Future Work 64

6.1 Conclusions . . . . . . . . . . . . . . . . . . . . 64

6.2 Future Work . . . . . . . . . . . . . . . . . . . . . 65

$\begin{array}{ll}\text { Bibliography } & 67\end{array}$ 


\section{List of Figures}

1.1 Typical data collection geometry of synthetic aperture radar (image courtesy of Sandia National Laboratories). . . . . . . . . . . . . 2

1.2 A SAR image containing two moving targets which are improperly imaged with conventional SAR processing that assumes a static scene [1]. 3

2.1 Frequencies allocated for radar operation (radartutorial.eu). $\ldots \ldots .7$

2.2 Stripmap mode SAR imaging geometry. . . . . . . . . . . . . . . 8

2.3 Spotlight mode SAR imaging geometry. . . . . . . . . . . . . . 10

2.4 Flowchart of the alternating direction method (ADM) for the LRSD. . 21

2.5 Non-overlapping subapertures. . . . . . . . . . . . . . . . . . . 22

2.6 Subaperture images of a synthetic SAR scene containing three static targets. (a) Ground truth image. (b) First subaperture image. (c) Second subaperture image. . . . . . . . . . . . . . 23

2.7 Subaperture images of a synthetic SAR scene containing two static targets and a moving target (in the scene center). (a) Conventional reconstruction (with quadratic phase errors due to a moving target). (b) First subaperture image. (c) Second subaperture image. . . . . . . . . 23

3.1 Block Diagram of the proposed moving target imaging approach. . . . 25 
4.1 Results of the synthetic scene experiment. (a) Ground truth scene indicating the locations of three moving targets in a noisy background and low SCR. (b) Conventionally reconstructed scene with quadratic phase errors due to the targets' motions. (c) Focused image produced by the SDF algorithm without LRSD (d) First subaperture image with a reduced azimuth resolution. (e) Second subaperture image with a reduced azimuth resolution. (f) Sparse image after LRSD, corresponding to the first subaperture. (g) Full resolution conventional image of the sparse part containing the moving targets. (h) Focused moving target image after LRSD and sparsity-driven focusing. (i) Background image after LRSD and the full-resolution conventional reconstruction. . . . . . .

4.2 Results of the real SAR scene experiment. (a) Ground truth scene indicating the locations of three moving targets in a real SAR background. Four strong static points were also added near the corners of the scene. (b) Conventionally reconstructed scene with quadratic phase errors due to the targets' motions. (c) Focused moving target image after processing by the proposed framework and subsequent sparsity-driven focusing. (d) Background image after LRSD and full-resolution conventional reconstruction. . . . . . . . . . . . . . . . .

4.3 Signal to clutter ratio (SCR) vs. mean squared error (MSE) performance plots using the proposed approach (SLRSD+SDF, red) and the sparsity-driven focusing only (SDF, black). The standard deviation of each measurement is given by an associated error bar. . . . . . . . . . 38

4.4 Signal to clutter ratio (SCR) vs. structural similarity index (SSIM) of the reconstructed SAR image for the proposed approach (red) and sparsitydriven focusing only (black). . . . . . . . . . . . . . . . 
4.5 Velocity experiment results. (a) Conventional moving target image (Velocity $=1 \mathrm{~m} / \mathrm{s}$, Phase error $=0.5 \pi$ radians) (b) Full resolution conventional image of the sparse component extracted by SLRSD. (c) Moving target image after SLRSD+SDF. (d) Conventional moving target image (Velocity $=3 \mathrm{~m} / \mathrm{s}$, Phase error $=1.5 \pi$ radians). (e) Full resolution conventional image of the sparse component extracted by SLRSD. (f) Moving target image after SLRSD+SDF. . . . . . . . . . . 42

4.6 SCR vs. MSE plots for various number of subapertures $(02,04,08) \ldots \quad 43$

4.7 SCR vs. MSE plots for 02 and 04 subapertures. . . . . . . . . . . . 44

4.8 SCR vs. MSE plots for various number of subapertures $(02,04,08)$ on a $\operatorname{logarithmic}$ scale. . . . . . . . . . . . . . . . . . 44

5.1 EMISAR water/bridge scene, Storebaelt, Denmark. Yellow boxes indicate some regions of interest for moving target imaging experiments. . .

5.2 (a) A larger view of the moving target scene 1 where a ship is highlighted by a red box. (b) Selected region containing the moving target. . . . . 50

5.3 Composite subaperture images of the moving target scene 1. (a) Subaperture image 1. (b) Subaperture image 2. (c) Subaperture image 3. (d) Subaperture image $4 \ldots \ldots \ldots$. . . . . . . . . . . . . .

5.4 Sparse subaperture images of the moving target scene 1. (a) Subaperture image 1. (b) Subaperture image 2. (c) Subaperture image 3. (d) Subaperture image $4 \ldots \ldots \ldots \ldots$. . . . . . . . . . . . . 5 . 52

5.5 Full-resolution reconstruction results of SLRSD for the moving target scene 1. (a) Full-resolution background SAR image. (b) Full-resolution sparse SAR image. (c) Region-enhanced background SAR image. (d) Sparsity-driven focused SAR image. . . . . . . . . . . . . . . .

5.6 (a) A larger view of the moving target scene 2 , where a region containing two moving targets is highlighted by a red box. (b) Selected part of the SAR image containing the moving targets. . . . . . . . . 55 
5.7 Full-resolution reconstruction results for the moving target scene 2. (a) Full-resolution background SAR image. (b) Full-resolution sparse SAR image. (c) Region-enhanced background SAR image. (d) Sparsity-driven focused SAR image. . . . . . . . . . . . . . . . . 56

5.8 Amplitude reflectivity profiles at various processing stages for the moving target scene 2 (First moving target, range bin $=29$ ). (a) Composite signal $(\mathrm{B}+\mathrm{S}$, green) and the Low-rank/background component (blue). (b) Sparse component (black). (c) SDF focused component(blue). . . . 57

5.9 Amplitude reflectivity profiles at various processing stages for the moving target scene 2 (Second moving target, range bin =94) (a) Composite signal $(\mathrm{B}+\mathrm{S}$, green) and the Low-rank/background component (blue). (b) Sparse component (black). (c) SDF focused component(blue). . . . 58

5.10 (a) A larger view of the moving target scene 3. A large vessel is highlighted by the red box. (b) Selected part of the SAR image containing

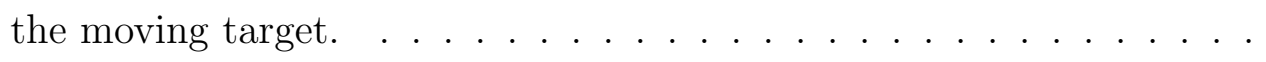

5.11 Composite subaperture images of the moving target scene 3. (a) Subaperture image 1. (b) Subaperture image 2. (c) Subaperture image 3. (d) Subaperture image $4 \ldots \ldots \ldots \ldots \ldots$. . . . . . . . . .

5.12 Full-resolution reconstruction results for the moving target scene 3. (a) Full-resolution background image. (b) Full-resolution sparse image which mostly contains the moving target. . . . . . . . . . . 62

5.13 Point and region enhanced reconstructions for the moving target scene 3. (a) Region-enhanced background image. (b) Point-enhanced moving target image. . . . . . . . . . . . . . . . . . . 62

5.14 Sparsity-driven focusing results for the moving target scene $3 . \ldots 63$ 


\section{List of Tables}

4.1 System parameters used for the target velocity simulations. . . . . . . . 41

4.2 CPU specifications for SLRSD experiments . . . . . . . . . . 45

4.3 Processing time and memory usage . . . . . . . . . . . . . . . . 45

5.1 EMISAR system parameters. . . . . . . . . . . . . . . . . . 48 


\section{Chapter 1}

\section{Introduction}

This thesis presents a new approach for synthetic aperture radar (SAR) imaging of moving targets. In this chapter we provide: 1) an introduction to SAR and the moving target imaging problem, 2) the motivation for this thesis and a brief description of the contributions, and 3) an outline of the thesis.

\subsection{Problem Definition and Motivation}

Synthetic aperture radar (SAR) imaging has gathered great significance in the last few decades for remote sensing of man-made as well as natural land features. SAR imagery offers distinct benefits over the optical counterpart [2]. Firstly, SAR is an active sensor having its own illumination that allows it to work equally well at day and night. Secondly, it is an all weather sensor. The electromagnetic waves at commonly used radar frequencies can penetrate fog, clouds and precipitation. Thirdly, the radio waves are scattered in an exclusive manner from different types of objects leading to some extra information about the area of interest. In this way, SAR imagery augments the information provided by the optical sensors for enhanced feature discrimination. A typical SAR data collection geometry is depicted in Figure 1.1.

Basic SAR processing assumes a stationary scene where all phase changes in the received signal correspond to the platform motion. Nevertheless, moving targets yield

additional phase errors due to motion during their exposure by the radar beam. This leads to their defocussing and misplacement in the overall reconstructed SAR image. To 


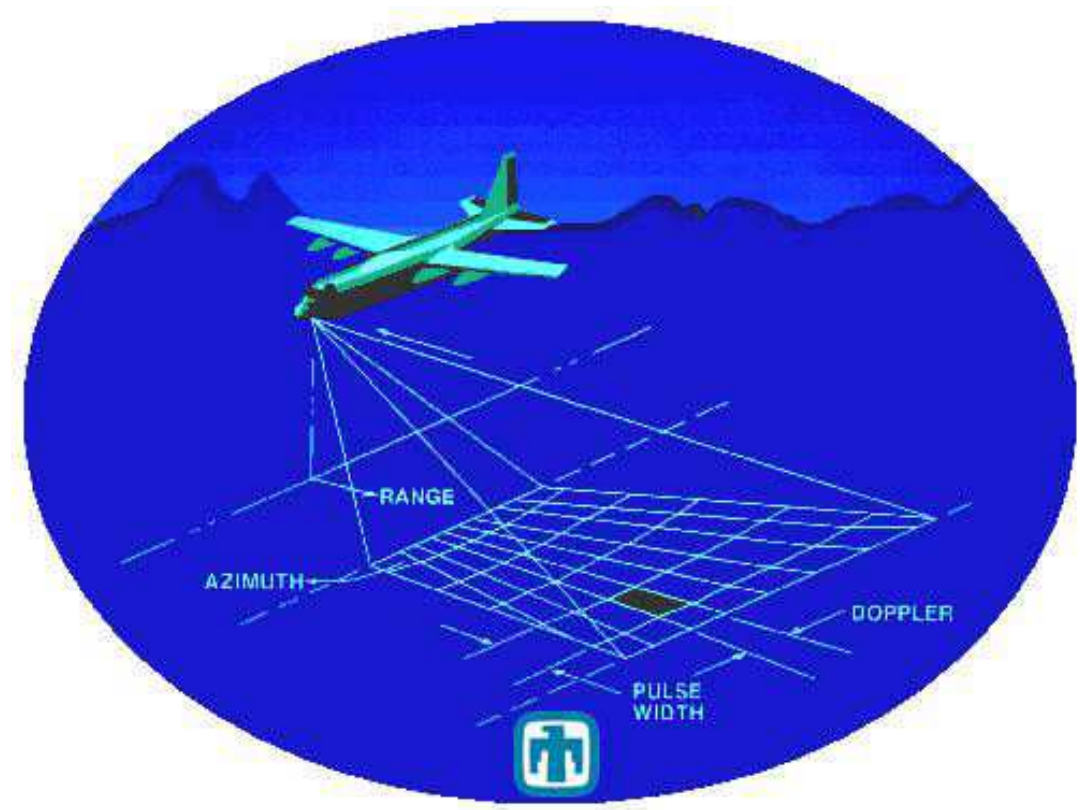

Figure 1.1: Typical data collection geometry of synthetic aperture radar (image courtesy of Sandia National Laboratories).

provide an example, Figure 1.2 highlights the image reconstruction artifacts produced by two moving targets in a largely static SAR scene. Moving target imaging with SAR primarily necessitates the detection of these targets from a mostly static background. Subsequent processing comprises of motion parameter estimation and focusing to the original positions based on phase history data.

For monostatic single antenna SAR systems, filtering based approaches are extensively employed as conventional methods of background/clutter suppression $[3,4,5,6]$. However, performance in these cases is heavily dependent on system parameters including pulse repetition frequency, platform velocity and azimuth compression ratios. The detection of slow moving targets is challenging and in most cases a very limited detection window is available. Time-frequency methods have also been suggested as in $[7]$.

Many modern day SAR systems incorporate multiple antennas for a robust phasebased detection of moving targets especially at low velocities [8, 9]. Space-time adaptive processing (STAP) has also been utilized as in [10]. Nevertheless, these systems are 


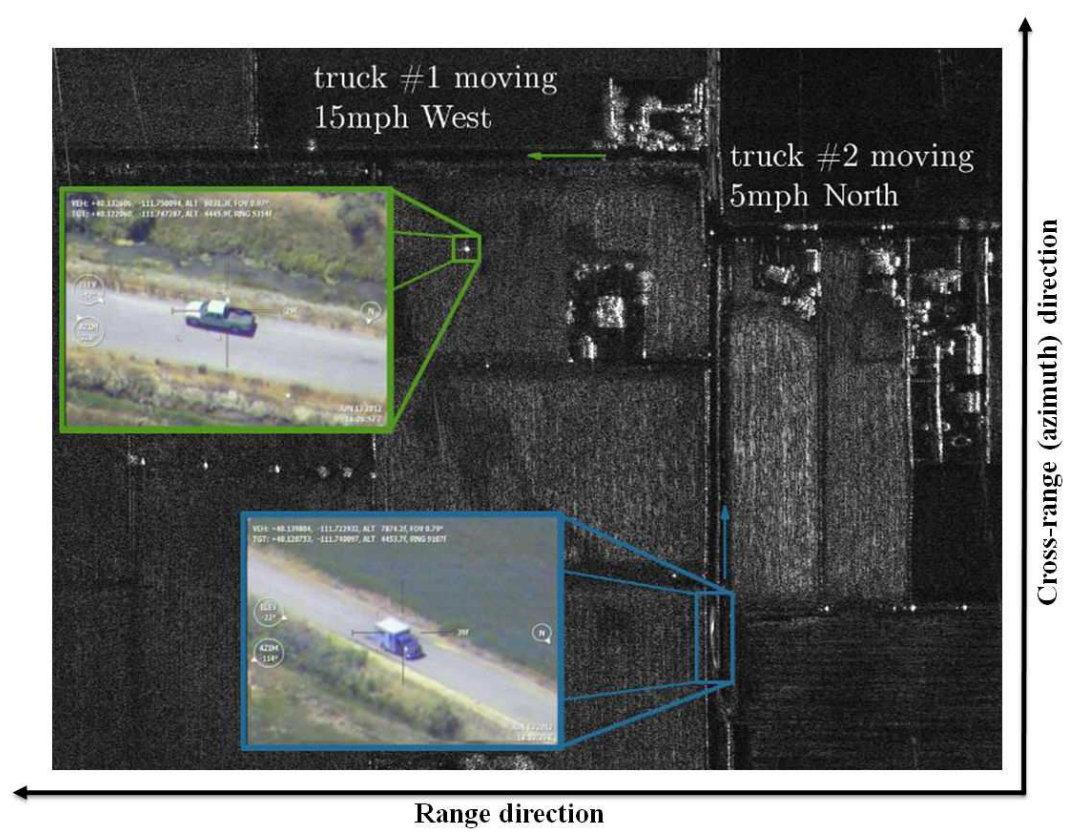

Figure 1.2: A SAR image containing two moving targets which are improperly imaged with conventional SAR processing that assumes a static scene [1].

more costly and sophisticated. This thesis focuses on single antenna SAR systems.

Sparsity-based methods have gathered great attention during the last decade by offering much better resolution (super-resolution in some cases $[11,12]$ ) and enhanced reconstruction quality compared to conventional SAR imaging [13, 14, 15, 16]. Some promising sparsity-based moving target imaging approaches have also been suggested as in $[17,18]$ which handle the additional phase terms caused by targets' motion as errors in the imaging model of a static SAR scene. However, these methods assume a comparatively high signal to clutter ratio (SCR) for best performance. Owing to the limitations of most practical SAR systems in producing high SCR data, detection of moving targets by clutter suppression is indispensable before applying these methods.

Low-rank and sparse decomposition (LRSD) is an effective technique for background and foreground separation attracting applications in various domains. For instance, in magnetic resonance imaging (MRI), it has been extensively applied for the decomposition of dynamic yet sparse innovations from a slowly varying background [19, 20]. Moreover, LRSD has found significant applications in computer vision [21]. It has also 
been employed for SAR image reconstruction [22, 23]. Some preliminary results for SAR moving target imaging using LRSD and backprojection have been reported in $[24,25,26,27]$. However, they do not provide a performance analysis for very low signal to clutter ratio (SCR) situations. In most cases, the decomposition is carried out on magnitude images and the recovery of complex phase information is not considered. Moreover, they do not suggest a solution to the azimuth resolution degradation that might originate due to the limited angles used for the backprojection.

The LRSD framework requires the construction of an input data matrix where every column corresponds to a temporal image of the same scene. For some applications like camera videos, these temporal images are directly accessible as movie frames. However for SAR, multiple frames of the same scene can be generated by splitting a complete azimuth aperture into a number of subapertures at the expense of a reduced azimuth resolution. Moving targets change their position in the different subapertures which facilitates their detection as a sparse component.

\subsection{Contributions made by this Thesis}

This thesis presents a new framework and an associated algorithm for LRSD-based moving target SAR imaging. This work contributes to the existing methods of moving target imaging with SAR in a number of aspects. Firstly, it expands the utility of sparsity-driven moving target imaging/focusing approaches [17] to very low SCR cases by offering a considerable gain in the SCR performance. Secondly, it enhances the general LRSD framework presented in [28] to the moving targets imaging problem without a constraint on the background to be spatially low-rank. Thirdly, a non-overlapping frequency domain SAR subaperture construction approach is devised which allows the recovery of full-resolution after the LRSD step using the decomposed subaperture data.

The full-resolution moving target image can be further processed by any suitable focusing, motion estimation algorithm. In this work we demonstrate the performance with sparsity-driven focusing (SDF). Additionally, we produce a final reconstruction of the full-resolution background image through region-enhanced processing [13]. Some 
preliminary results of this work have been presented in [29].

\subsection{Organization of the Thesis}

The remainder of this thesis is organized as follows: Chapter 2 provides a coverage of the preliminary topics for the SAR moving target imaging problem. The proposed method is formulated in Chapter 3. Experimental results with synthetic as well as semisynthetic data (using MiniSAR background) are provided in Chapter 4. Furthermore, a thorough analysis of important performance parameters based on numerical simulations is presented. Finally in Chapter 5, experimental results are demonstrated with EMISAR data containing actual moving targets. We stipulate the conclusions drawn from this thesis in Chapter 6 and discuss the possible future research dimensions. 


\section{Chapter 2}

\section{Background}

This chapter provides the necessary background for our proposed framework. Firstly, we describe some basics of SAR and moving target imaging. Secondly, we introduce regularization based image reconstruction and its benefits over conventional SAR processing. Thirdly, we delineate some details about sparsity-driven SAR moving target imaging and the LRSD decomposition. Finally, we explain the significance of subaperture processing and present a small experiment to illustrate the response of moving targets in the subaperture images.

\subsection{SAR Basics}

First radar was developed in 1930s in the mist of the world war where it proved to be an extremely useful instrument for air defense. The word "Radar" stands for Radio Detection and Ranging. A conventional radar transmits radio frequency (RF) in the form of a high energy pulse towards the area of interest and waits for the echoes from potential targets. By measuring the round-trip time $T$ of the RF pulse, radar is able to meausure the distance/range $R$ of the target.

$$
R=\frac{c \times T}{2}
$$

where $c$ is the speed of light $\left(\approx 3 \times 10^{8} \mathrm{~m} / \mathrm{s}\right)$. The commonly used frequencies for radar operation are illustrated in Figure 2.1.

The ability to resolve two closely spaced targets as separate objects depends on the range and azimuth resolutions of the radar. The range resolution $\rho_{r}$ is defined by the 


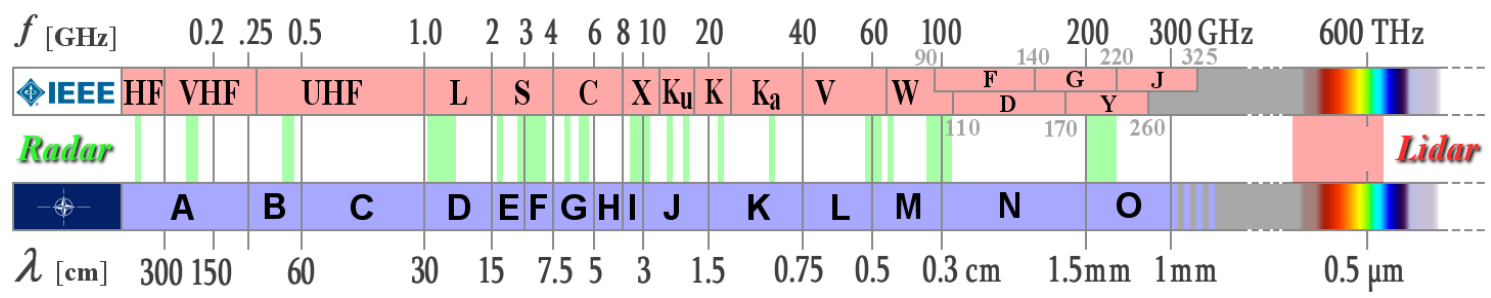

Figure 2.1: Frequencies allocated for radar operation (radartutorial.eu).

pulse-width $t_{p}$ as follows:

$$
\rho_{r}=\frac{c t_{p}}{2}
$$

Since the bandwidth $B$ of the transmitted pulse is the reciprocal of pulse-width, we have:

$$
\rho_{r}=\frac{c}{2 B}
$$

Usually radars are very accurate in the measurement of range. Modern day radars employ advanced techniques such as pulse compression $[30,31]$ to achieve very fine range resolution despite transmitting very long pulses. This is achieved by artificially increasing the bandwidth of the transmitted pulses by phase or frequency modulations. The received pulses are subsequently compressed in the radar receiver by a matched filtering operation. In contrast to the range resolution, the cross-range or azimuth resolution $\rho_{a}$ of a conventional beam steering radar is largely dependent on the azimuth antenna beam-width which is defined by the effective antenna length $L_{e f f}$.

$$
\rho_{a}=\frac{R \lambda}{L_{e f f}}
$$

where $\lambda$ is the radar wavelength corresponding to the transmission frequency $f$ ( $\lambda=$ $C / f)$. The relation between $\rho_{a}$ and the effective antenna length demands a very long antenna to achieve a fine azimuth resolution which is comparable to the typical range resolution $\rho_{r}$. For example, at $16 \mathrm{GHz}$ frequency $(\lambda \approx 2 \mathrm{~cm})$ and $20 \mathrm{~km}$ range, a radar would require an antenna length of $375 \mathrm{~m}$ to achieve an azimuth resolution of $1 \mathrm{~m}$. Such antenna lengths are not practical for most applications. Synthetic aperture radar solves this problem by utilizing the platform motion to synthesize a very long antenna. Since a point target is illuminated by a side-looking SAR for an extended period of 


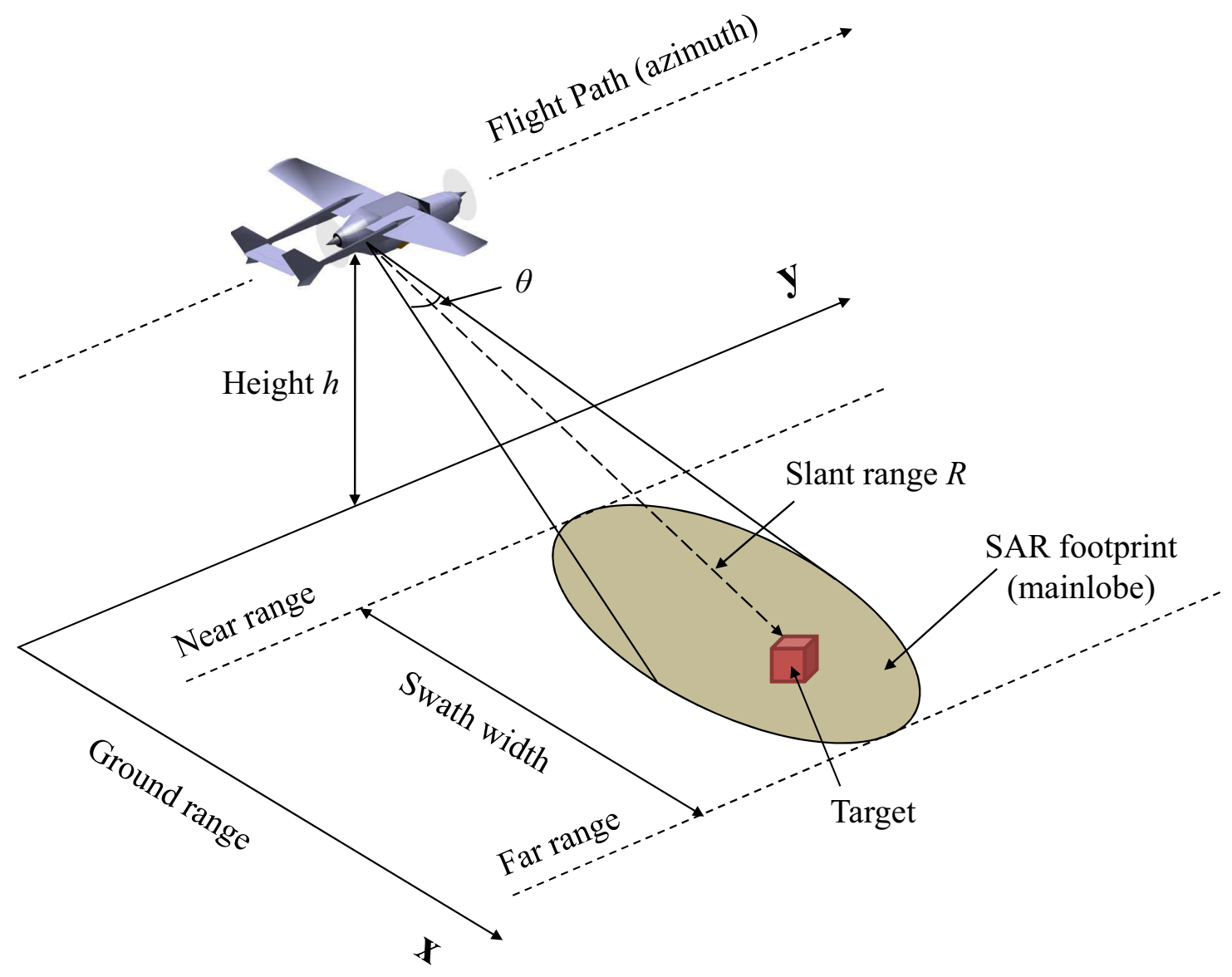

Figure 2.2: Stripmap mode SAR imaging geometry.

time, the data collected in this interval are coherently processed in the SAR receiver to achieve extremely fine azimuth resolution. This enables SAR to construct very high resolution radar reflectivity images of a desired area. An additional feature of SAR imagery is its availability during day and night because it is an active sensor with its own illumination. Moreover, SAR can operate in all weather conditions including rain, fog and dust. SAR waves at commonly used frequencies can pass through a cloud cover which is not possible with optical imaging. These unique features have established SAR as an indispensable sensor for remote sensing applications. 


\subsubsection{SAR Imaging Modes}

SAR can operate in a variety of modes most of which differ in terms of the imaging geometry, image processing and final application. Two most commonly used modes are the stripmap mode and the spotlight mode.

In stripmap mode, the SAR antenna stays fixed in a side-looking position and a scan of the area under illumination is generated in the form of a strip. This is due to linear motion of the platform which ideally follows a straight line except for small motion errors. A typical geometry of stripmap mode SAR is exhibited in Figure 2.2.

In spotlight mode, the SAR antenna focuses on a selected area on the ground while the antenna keeps on steering as the platform moves to consistently illuminate the same area. The platform can follow an arbitrary path but mostly a linear or circular platform motion is used in practice. Owing to its extremely extended illumination times and data collection at a wide range of angles, this mode can be used to reconstruct unmatched fine resolution imagery of the area under observation. Figure 2.3 depicts the spotlight mode imaging geometry.

\subsubsection{Imaging Model for the Spotlight Mode}

A tomographic formulation of the spotlight mode SAR imaging model was presented in [32]. The transmitted signal by a SAR system usually consists of a linear frequency modulated (LFM) pulse defined as:

$$
s(t)= \begin{cases}e^{j\left(w_{0} t+\alpha t^{2}\right)} & |t| \leq \frac{t_{p}}{2} \\ 0 & \text { otherwise }\end{cases}
$$

where $w_{0}$ is the transmission frequency, $t_{p}$ is the pulse-width, and $2 \alpha$ is the LFM chirp rate. Consider a complex-valued reflectivity field $f(x, y)$ of radius $L$ centered at the origin $(0,0)$ of the imaging grid (Figure 2.3). Owing to the continuous pointing of the SAR antenna, the low-pass filtered observation corresponding to an angle $\theta$ can be modeled as:

$$
r_{\theta}(t)=\iint_{x^{2}+y^{2} \leq L^{2}} f(x, y) e^{-j \Psi(t)(x \cos \theta+y \sin \theta)} d x d y
$$




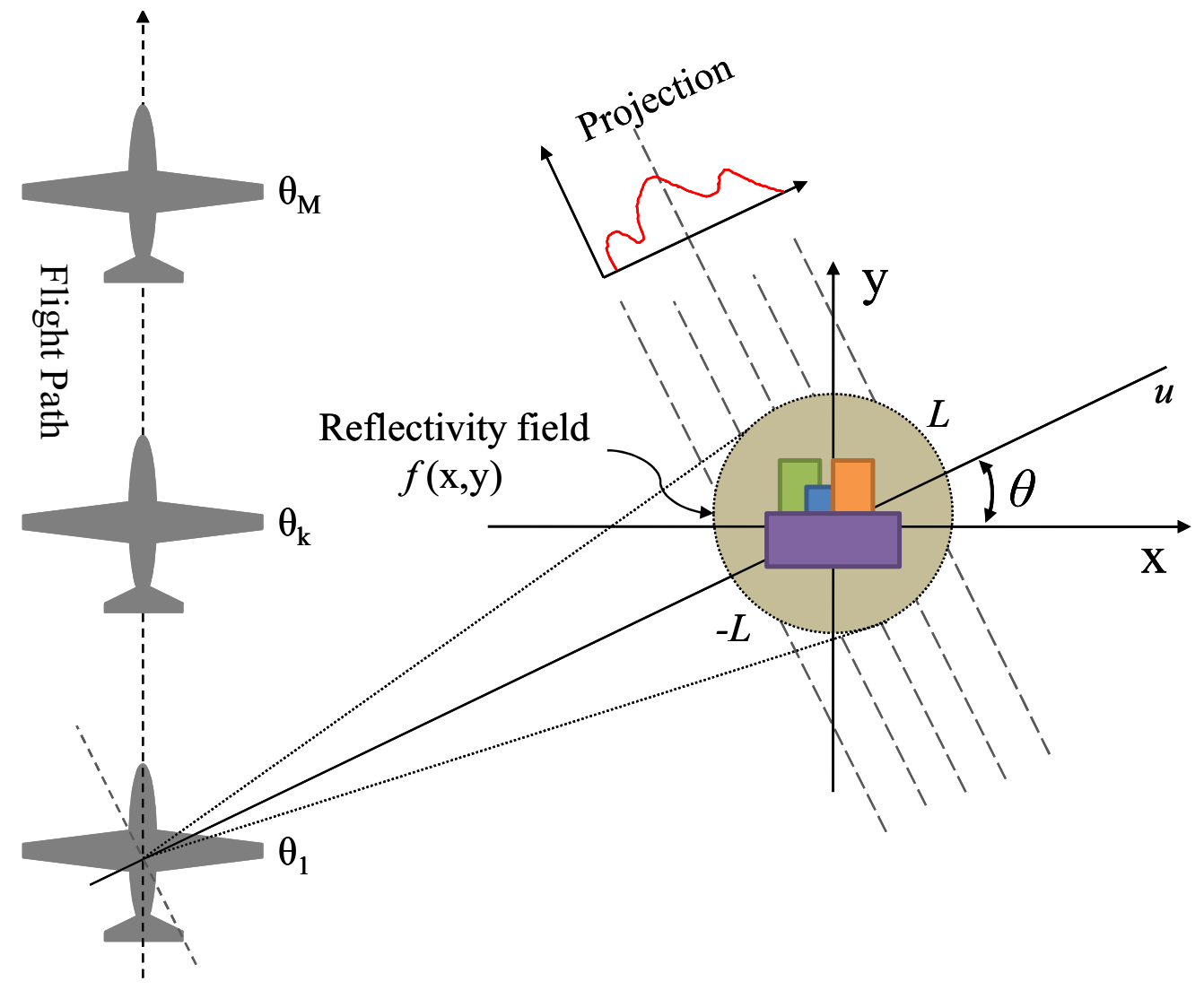

Figure 2.3: Spotlight mode SAR imaging geometry.

where $\Psi(t)=\frac{2}{c}\left(w_{0}+2 \alpha\left(t-2 \frac{R_{\theta}}{c}\right)\right)$ is the radial spatial frequency and $R_{\theta}$ denotes the range to scene center at the observation angle $\theta$. The continuous observation model of (2.6) can be discretized for an arbitrary total number of $M$ observation steps:

$$
\underbrace{\left[\begin{array}{c}
r_{1} \\
r_{2} \\
r_{M}
\end{array}\right]}_{r}=\underbrace{\left[\begin{array}{c}
C_{1} \\
C_{2} \\
C_{M}
\end{array}\right]}_{C} f
$$

where $r$ comprises of the phase history data of the reflectivity field $f$ and $C$ is a discrete observation model of the SAR system. 


\subsection{Moving Target Imaging with SAR}

A moving target produces phase errors in the SAR observed data which may cause defocussing and shift of position in the final reconstructed image. The phase perturbations due to the moving target can be observed as a slowly varying carrier phase from one pulse to another. As a consequence, these phase modulations mostly appear in the cross-range direction. Since SAR primarily uses the cross-range Doppler signals to attain a high azimuth resolution, the phase perturbations due to target motion interact with the normal SAR imaging. This interaction needs to be exploited for the detection and imaging of moving targets in mostly stationary SAR backgrounds.

A SAR signal from a target moving in azimuth direction [3] can be described as:

$$
m(\eta) \cong a\left[\left(V_{p}-v_{a}\right) \eta\right] \exp \left(j \frac{2 \pi}{\lambda R_{0}} V_{p}^{2} \eta^{2}\left(1-\frac{v_{a}}{V_{p}}\right)^{2}\right)
$$

where $\eta$ is the azimuth time (usually named as the slow time), $V_{p}$ is the SAR platform velocity, $R_{0}$ is the range of closest approach and $v_{a}$ represents the azimuth velocity of the target. $a($.$) includes the azimuth weighting, target reflectivity f$ and the constant phase terms. The quadratic phase error due to cross-range velocity of the target [33] can be approximated as:

$$
\phi(\eta)=\frac{4 \pi v_{a} V_{p} \eta^{2}}{\lambda R_{0}}
$$

A moving target in the SAR scene can have both range and cross-range velocity components. The range component of the velocity causes smear and defocussing simultaneously in the range and cross-range directions. Additionally, it causes a cross-range position shift. On the other hand, the cross-range component of the target velocity produces a defocussing in the cross-range direction only. In both cases, the defocussing is related to the phase errors in the observed SAR data.

Conventionally, two commonly used techniques for moving target detection are frequency based detection and phase based detection. 


\subsubsection{Frequency based Moving Target Detection}

Frequency detection relies on the excessive Doppler frequency generated by a moving target as compared to the clutter (static points in the scene). However, this necessitates the moving target Doppler shift to exceed the width of the Doppler spectrum produced by the clutter. The overall detectability window is typically very small which is limited by several factors including the pulse repetition frequency (PRF), system bandwidth, antenna beam-width in azimuth and the platform velocity. Slow-moving target detection is particularly challenging with this approach. The lower limit on the radial velocity $v_{r}$ of the target for frequency based detection is as follows [3]:

$$
v_{r}>\frac{\gamma V_{p} \lambda}{2 \rho_{a}}
$$

where $\gamma$ is a threshold chosen based on the desired probabilities of detection and false alarm.

\subsubsection{Phase based Moving Target Detection}

Very slow moving targets can be detected by phase based detection where the change in the received signal phase due to target motion is observed instead of the Doppler frequency shift. For this, usually two receive antennas are incorporated to record two coherent observations of the target with a small time difference $\Delta t$ due to antenna phase center separation $D$. The difference signal $m_{\Delta}(x)$ corresponding to the two observations (which are very similar except for the phase term) [3] is described as:

$$
m_{\Delta}(x)=m_{1}(x)\left[1-e^{j(4 \pi / \lambda) v_{r} \Delta t}\right]
$$

where $x=V_{p} t$ is the azimuth spatial coordinate and $m_{1}$ is the signal received by the first antenna. Furthermore, the magnitude of difference signal is given as:

$$
\left|m_{\Delta}(x)\right|=2\left|m_{1}(x)\right|\left|\sin \left(\frac{2 \pi v_{r} \Delta t}{\lambda}\right)\right|
$$

where the constant phase terms have been omitted. Since the clutter is highly correlated in the two observations, it is mostly canceled out. The performance of phase based moving target detection is much improved as compared to the frequency based detection but it demands extra system complexity and cost. 


\subsection{Regularization based Imaging}

The image reconstruction and restoration problems can be seen as solving for the unknown original image $f$ (ground truth image in case of SAR) given knowledge of the observed data $g$ and the system model $H$. In most cases, the problem formulation includes the observation noise $q$ as given below [34]:

$$
g=H f+q
$$

At a first glance, a matrix inverse seems to provide a solution to this problem but unfortunately this naive approach does not produce usable solutions. There are various possible reasons to this: 1) an exact solution may not exist due to noise, 2) a solution may not be unique due to nonempty null space of $H$ or in other words, many possible $f$ could produce the same observed data $g$, and 3) instability of the solution in presence of small perturbations in the observed data. Moreover, no prior information about $f$ is used which leads to poor solutions.

\subsubsection{Least-squares Solution and Regularization}

A common approach to mitigate the existence problem is to find a least-squares solution. In particular, it tries to estimate the solution that best fits to the observed data in terms of least-squares by solving the unconstrained optimization problem:

$$
\widehat{f}_{L S}=\arg \min _{f}\|g-H f\|_{2}^{2}
$$

where $\|.\|_{2}^{2}$ represents the $l_{2}$-norm. The closed-form solution to the least-squares problem is as follows:

$$
\left(H^{T} H\right) \widehat{f}_{L S}=H^{T} g
$$

The non-uniqueness problem is typically dealt by seeking a generalized solution among the available least-squares solutions based on minimum energy function. However, there are some serious shortcomings associated with these approaches. Firstly, the image components pertaining to the null space of $H$ (unobserved in the data) are not properly reconstructed. Secondly, the generalized solutions are very sensitive to 
perturbations in the observed data which may lead to unstable solutions even in the presence of small observation noise.

A more practical approach that overcomes above mentioned limitations is to incorporate prior information about $f$ as an extra regularization term. In general:

$$
\widehat{f}=\arg \min _{f} \Phi_{\text {data }}(f, g)+\lambda \Phi_{\text {prior }}(f)
$$

where $\Phi_{\text {data }}(f, g)$ is called the data fidelity term which enforces the final solution to be consistent with the observed data. $\Phi_{\text {prior }}(f)$ is the prior information about $f . \lambda$ is a regularization parameter that balances the two terms. Here, we provide a brief description of few widely used regularization schemes.

\subsubsection{Quadratic Regularization}

Quadratic regularization schemes include an extra quadratic term to incorporate the prior information. A well known quadratic regularization approach is the Tikhonov regularization which is formulated as follows: $[35,36]$

$$
\widehat{f}_{T i k}=\arg \min _{f}\|g-H f\|_{2}^{2}+\lambda\|L f\|_{2}^{2}
$$

where $L$ is is the regularization operator which could be provided in the form of a matrix. In some applications, $L$ could be a gradient operator enforcing smoothness in the final solution which is very useful for noise suppression. The extra regularization term aims to stabilize the the generalized solution by means of the additional quadratic penalty. The closed-form solution for the Tikhonov type regularization is given by:

$$
\left(H^{T} H+\lambda L^{T} L\right) \widehat{f}_{T i k}(\lambda)=H^{T} g
$$

\subsubsection{Non-quadratic Regularization}

The quadratic regularization models are computationally efficient since they lead to an inverse filter that is a linear function of the observed data (2.18). However, more powerful reconstruction results could be achieved if non-quadratic regularization is permissible. As an example, high frequency noise suppression with quadratic regularization 
results in loss of high frequency content (e.g., sharp edges) in the final image. A widely adopted non-quadratic regularization model for image processing applications is a total variation (TV) based formulation [37, 38]:

$$
\widehat{f}_{T V}=\arg \min _{f}\|g-H f\|_{2}^{2}+\lambda\|\nabla f\|_{1}^{1}
$$

where $\nabla$ is a $2 \mathrm{D}$ gradient operator. This type of regularization preserves strong edges and produces improved reconstruction quality in piece-wise smooth regions.

Non-quadratic regularization has received great significance for sparsity-based imaging in various domains. Consider an imaging problem that involves a sparse field with only few non zero components. Alternatively, one could exploit the fact that most images have a sparse representation in some transform domain. This is a typical scenario for SAR imaging where non-quadratic regularization can produce very improved results with greater energy concentration as compared to the quadratic regularization [13]. A variety of non-quadratic constraints could be used including the $l_{p}$-norms defined as:

$$
\|f\|_{p}=\left(\sum_{i=1}^{N}\left|f_{i}\right|^{p}\right)^{1 / p}
$$

Generally, $l_{p}$-norm constraints with $p<2$ provide sparse solutions.

\subsection{Feature-enhanced SAR Image Reconstruction}

A feature-enhanced SAR image reconstruction approach based on non-quadratic regularization was proposed in [13]. The overall SAR image formation in this case is modeled by the following optimization problem:

$$
\hat{f}=\arg \min _{f} J(f)=\arg \min _{f}\|g-H f\|_{2}^{2}+\lambda_{1}\|f\|_{p}^{p}+\lambda_{2}\|D|f|\|_{p}^{p}
$$

where $\|.\|_{p}^{p}$ is the $l_{p}$-norm and $D$ is the two dimensional discrete derivative (gradient) operator. $|f|$ defines the magnitude vector of the complex reflectivity field $f$. Relative magnitudes of $\lambda_{1}$ and $\lambda_{2}$ define the balance between point-based and region-based feature enhancement. 


\subsubsection{Point-enhanced Reconstruction}

With $p \leq 1$ the term $\|f\|_{p}^{p}$ in $(2.21)$ enforces an energy-type constraint where the sparse features (dominant scatterers) are enhanced in the solution. This results in an improved resolution for the point features where other image artifacts are relatively suppressed.

\subsubsection{Region-enhanced Reconstruction}

Region-based enhancement is also desirable in SAR images, especially for speckle reduction in constant or slowly varying background regions. Additionally, it can lead to improved image segmentation at the post-processing stages. The third term in (2.21)

$\left(\|D|f|\|_{p}^{p}\right.$ with $\left.p \leq 2\right)$ dictates the minimization of $2 \mathrm{D}$ gradient of the reflectivity field magnitudes $|f|$. Consequently, the solution with minimum gradient is a smooth regionenhanced SAR image.

\subsection{Sparsity-driven Moving Target Imaging}

A sparsity-driven framework was proposed in $[17,18]$ for SAR moving target imaging which treats the phase terms induced by the target motion as errors in the imaging model. Here, we provide an overview of their approach. The observed phase history $r$ for a typical SAR scene corresponds to the sum of the responses from every point scatterer in the scene.

$$
r=\underbrace{C_{c l m n-1} f(1)}_{r p_{1}}+\underbrace{C_{c l m n-2} f(2)}_{r p_{2}}+. .+. .+\underbrace{C_{c l m n-I} f(I)}_{r p_{I}}
$$

where $C$ is the model matrix with $C_{c l m n-i}$ being its $i-t h$ column. $r p_{i}$ denotes the SAR data related to the complex reflectivity $f(i)$ for the $i-t h$ point in the scene.

Consider a moving target at the $i-t h$ point of the scene with motion parameters leading to the cross-range defocusing. The observed SAR data from this target can be 
expressed as:

$$
\left[\begin{array}{c}
r p_{i_{1_{e}}} \\
r p_{i_{2_{e}}} \\
\cdot \\
\cdot \\
r p_{i_{M_{e}}}
\end{array}\right]=\left[\begin{array}{c}
e^{j \phi_{i}(1)} r p_{i_{1}} \\
e^{j \phi_{i}(2)} r p_{i_{2}} \\
\cdot \\
\cdot \\
\cdot \\
e^{j \phi_{i}(M)} r p_{i_{M}}
\end{array}\right]
$$

where $\phi_{i}$ is the phase error due to the target's motion. $r p_{i}$ and $r p_{i_{e}}$ are the corresponding phase history data of the stationary and the moving target respectively. Similarly, this relation can also be defined for the model matrix $C$ as given below:

$$
\left[\begin{array}{c}
C_{c l m n-i_{1}}(\phi) \\
C_{c l m n-i_{2}}(\phi) \\
\cdot \\
\cdot \\
\cdot \\
C_{c l m n-i_{M}}(\phi)
\end{array}\right]=\left[\begin{array}{c}
e^{j \phi_{i}(1)} C_{c l m n-i_{1}} \\
e^{j \phi_{i}(2)} C_{c l m n-i_{2}} \\
\cdot \\
\cdot \\
e^{j \phi_{i}(M)} C_{c l m n-i_{M}}
\end{array}\right]
$$

where $C_{c l m n-i}(\phi)$ represents the $i$-th column of the model matrix $C(\phi)$ that incorporates the target motion. $C_{c l m n-i_{m}}(\phi)$ refers to the part of $C_{c l m n-i}(\phi)$ with reference to the $m-t h$ cross-range position. Taking the observation noise into consideration, the overall system's observation model could be defined as:

$$
g=C(\phi) f+v
$$

where $v$ denotes the additive noise in the observation process. This formulation is very useful since it allows the moving target imaging problem to be viewed as the stationary scene imaging problem with some additional phase errors. The objective is to estimate the complex reflectivity $f$ and the phase errors $\phi$ given the noisy observed data $g$.

A sparsity-driven approach can be applied for the simultaneous estimation of $f$ and $\phi$. In this context, a non-quadratic regularization framework is employed that incorporates the prior information about the sparse nature of the reflectivity field and the phase errors. A vector $\beta$ is introduced that accounts for the phase errors due to all 
points of the scene and corresponding to all aperture positions.

$$
\beta=\left[\begin{array}{c}
\beta_{1} \\
\beta_{2} \\
\cdot \\
\cdot \\
\cdot \\
\beta_{M}
\end{array}\right]
$$

where $\beta_{m}$ denotes the phase errors vector with respect to the $m-t h$ aperture position:

$$
\beta_{m}=\left[e^{j \phi_{1}(m)}, e^{j \phi_{2}(m)}, \ldots, e^{j \phi_{I}(m)}\right]^{T}
$$

The overall optimization problem aims at cost minimization for $f$ and $\beta$ and it is formulated as:

$$
\begin{array}{r}
\arg \min _{f, \beta} J(f, \beta)=\arg \min _{f, \beta}\|g-C(\phi) f\|_{2}^{2}+\lambda_{1}\|f\|_{1}+\lambda_{2}\|\beta-\mathbf{1}\|_{1} \\
\text { s.t. } \quad|\beta(i)|=1 \quad \forall i
\end{array}
$$

where $\lambda_{1}, \lambda_{2}$ are the regularization parameters and $\mathbf{1}$ denotes a vector of ones. The sparsity information related to the phase errors is included in the problem by the regularization term $\|\beta-\mathbf{1}\|_{1}$. Owing to the small number of moving targets in a typical SAR scene as compared to the stationary points, most phase error values are zero. Since $\beta$ values are defined in terms of $e^{j \phi}$, most values of $\beta$ are one.

The solution of this optimization problem is based on an iterative method described in [14]. First step is to solve for the reflectivity field $f$. The corresponding subproblem can be defined as:

$$
\hat{f}^{(n+1)}=\arg \min _{f} J\left(f, \hat{\beta}^{(n)}\right)=\arg \min _{f}\left\|g-C\left(\phi^{n}\right) f\right\|_{2}^{2}+\lambda_{1}\|f\|_{1}
$$

The estimated field $\hat{f}$ from this step is used in the second step for the phase error estimation. The subproblem in the second step requires minimization of the following cost function for every aperture position:

$$
\begin{array}{r}
\hat{\beta}_{m}^{(n+1)}=\arg \min _{\beta_{m}} J\left(\hat{f}^{(n+1)}, \beta_{m}\right)=\arg \min _{\beta_{m}}\left\|g_{m}-C_{m} T^{(n+1)} \beta_{m}\right\|_{2}^{2}+\lambda_{2}\left\|\beta_{m}-\mathbf{1}\right\|_{1} \\
\text { s.t. }\left|\beta_{m}(i)\right|=1 \quad \forall i
\end{array}
$$


where $n$ is the iteration number and $T$ is a diagonal matrix with $\hat{f}(i)$ being its diagonal values. $T$ can be defined as:

$$
T^{(n+1)}=\operatorname{diag}\left\{\hat{f}^{(n+1)}(i)\right\}
$$

The constrained subproblem in (2.30) is reformulated as an unconstrained optimization problem and solved in a similar manner to the field estimation step.

\subsection{Low-rank and Sparse Decomposition (LRSD)}

Recovery of the low-rank and sparse components of an arbitrary matrix has found a broad range of applications. It has been shown that under some reasonable assumptions, exact recovery of low-rank and sparse components is realizable by robust principal component analysis (RPCA) [39, 24, 40]. Similarly, the objective of the LRSD is to decompose a matrix $D$ into its low-rank $B$ and sparse $S$ components as follows:

$$
\min _{B, S} \operatorname{rank}(B)+\lambda\|S\|_{0} \quad \text { s.t. } \quad D=B+S
$$

where $\lambda$ is a regularization parameter. However, rank minimization requires the minimization of non-zero singular values which is an NP-hard problem. It was suggested by $[41,42,43]$ that a convex relaxation could be employed under some conditions by replacing the $\operatorname{rank}($.$) and l_{0}$-norm constraints with the nuclear-norm $\left(\|\cdot\|_{*}\right)$ and the $l_{1}$-norm, respectively. After considering these convex relaxations, the reformulated problem is given as:

$$
\min _{B, S}\|B\|_{*}+\lambda\|S\|_{1} \quad \text { s.t. } \quad D=B+S
$$

This problem has been efficiently solved in a variety of ways by using local optimization algorithms. A bilateral random projections (BRP) scheme was proposed in [44] to significantly accelerate the solution. A gradient descent based iterative scheme was proposed in [39] and an alternating direction method (ADM) was suggested for the augmented Lagrangian form of the problem in $[45,46]$. A flowchart of the typical processing steps for the LRSD based on ADM is given in Figure 2.4. The rank minimization 
(nuclear norm) is solved by singular value thresholding and the sparse component is resolved with a soft thresholding operation.

In addition to its extensive application in medical imaging and computer vision, LRSD framework has also been employed for SAR image reconstruction [22, 23]. Some preliminary results for the SAR moving target imaging with LRSD and backprojection have been reported in $[24,25,26,27]$.

\subsection{Subaperture Processing}

A point target remains illuminated by the SAR for an extended time defined by the overall imaging geometry and the system parameters including the platform velocity, antenna beam-width and look angle. The data received during the complete illumination time corresponds to a full azimuth aperture. Basic SAR processing utilizes the full aperture data to synthesize a large antenna achieving a fine spatial resolution. However, the full aperture data can be divided into multiple subapertures where each subaperture captures only a portion of the target's reflectivity and phase history [5, 2]. Nevertheless, in this process, the azimuth resolution is degraded by some factor. Figure 2.5 provides a conceptual view of the non overlapping subapertures on a SAR footprint. Subaperture processing has been widely employed for SAR image reconstruction $[47,48]$ multilook processing which is an efficient method for speckle reduction in SAR imagery. Generally, multilook processing employs multiple overlapping subapertures for improved performance $[49,50]$.

\subsubsection{Subaperture Imaging of Moving Targets}

Moving targets in a SAR scene behave differently in the subaperture images as compared to the static points. In particular, static points mainly lose their azimuth resolution so they appear spread in every subaperture image. Figure 2.6 describes the results of a simple experiment where a synthetic SAR scene consisting of three static point targets is used to generate subaperture images. The two subaperture images are shown in Figure 2.6(a \& b) where the most observable effect is spreading of the targets 


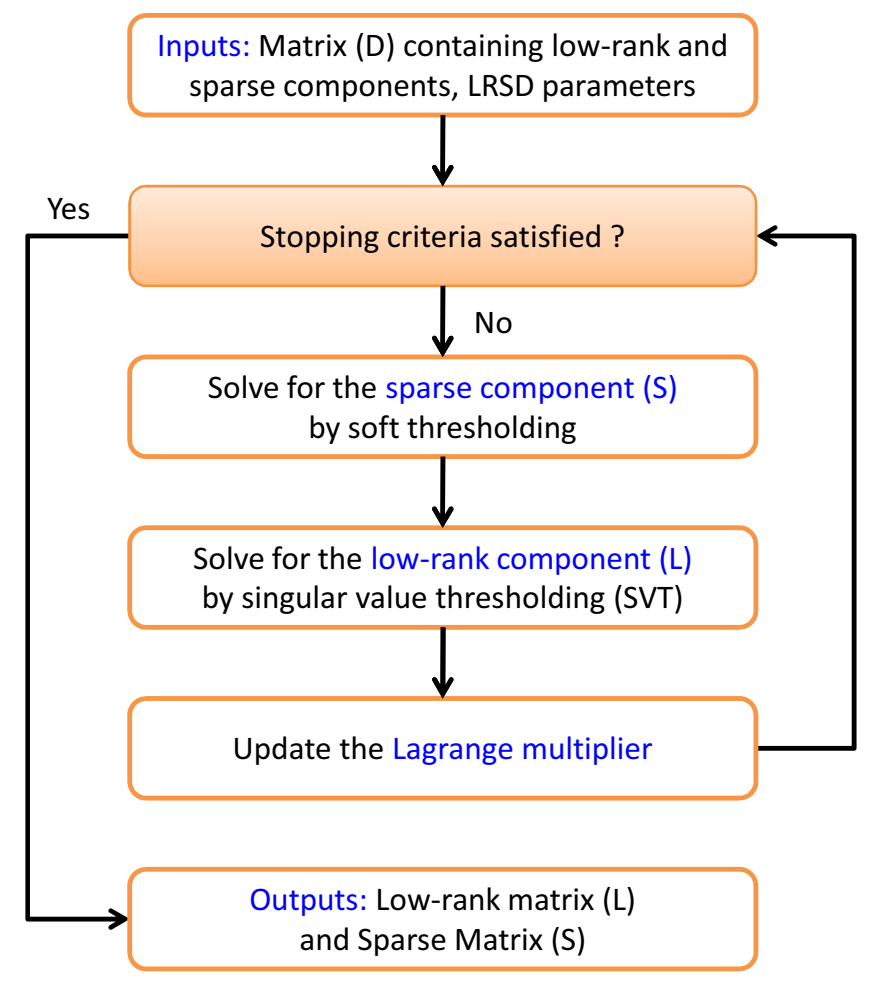

Figure 2.4: Flowchart of the alternating direction method (ADM) for the LRSD. 

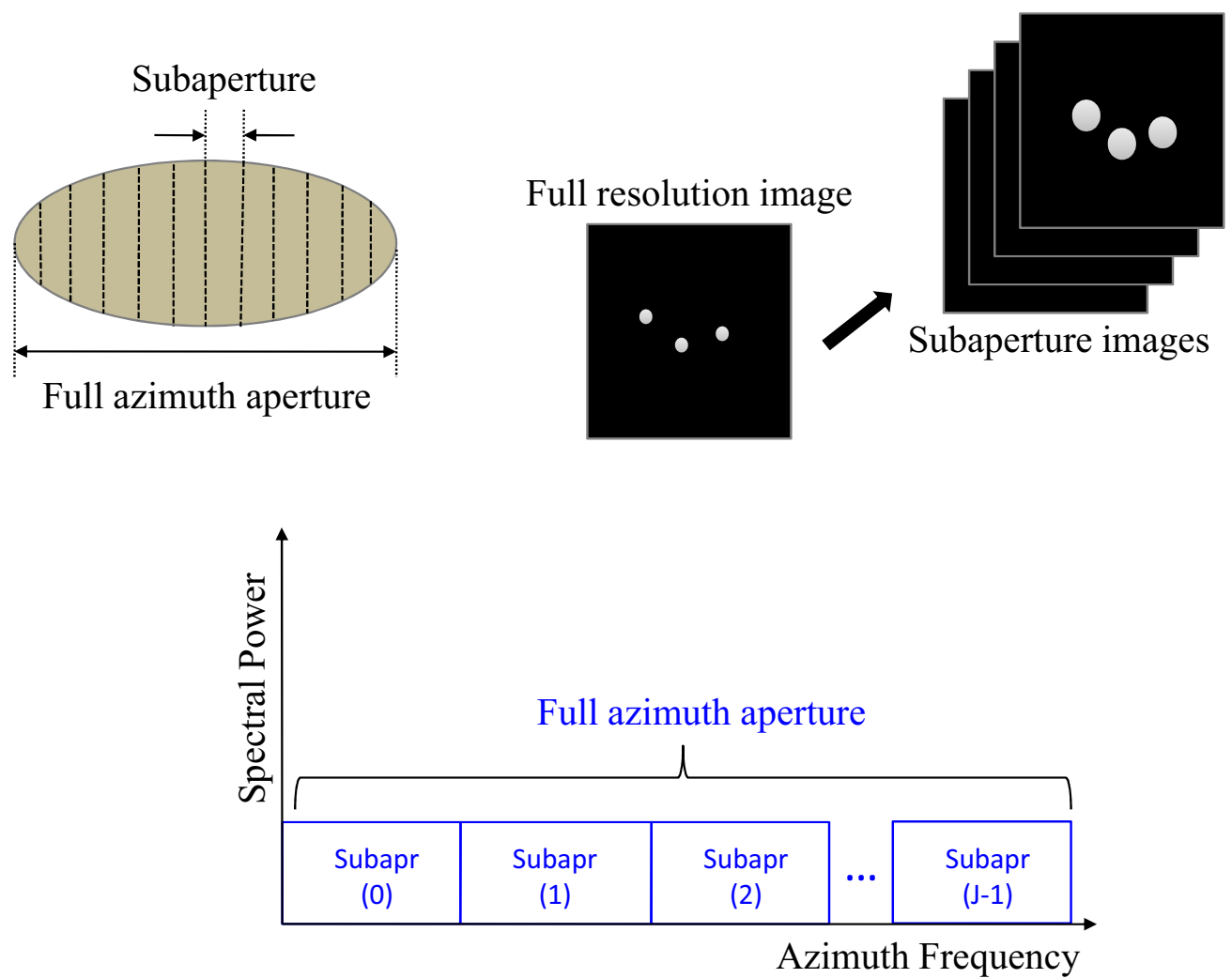

Figure 2.5: Non-overlapping subapertures.

in azimuth direction.

This experiment was repeated with one of the point targets (located in the scene center) having an azimuth motion. This motion was modeled by a quadratic phase error in the point target response. The other two targets are static as before. Figure 2.7 illustrates the impact of target motion in the subaperture images where in addition to the azimuth spread, a noticeable shift of the moving target's position across subapertures is observable. We exploit this useful property of the subaperture processing for our proposed moving target imaging approach. 


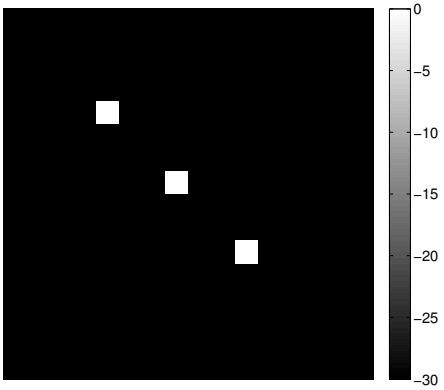

(a)

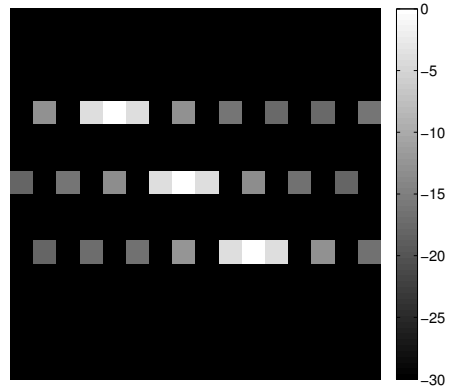

(b)

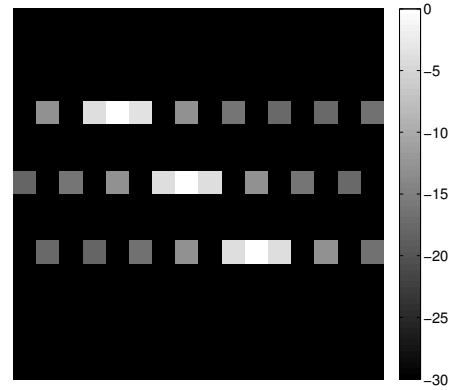

(c)

Figure 2.6: Subaperture images of a synthetic SAR scene containing three static targets.

(a) Ground truth image. (b) First subaperture image. (c) Second subaperture image.

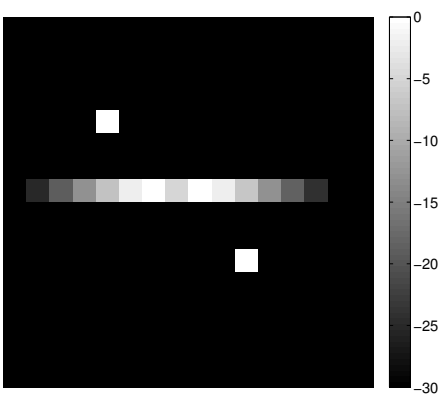

(a)

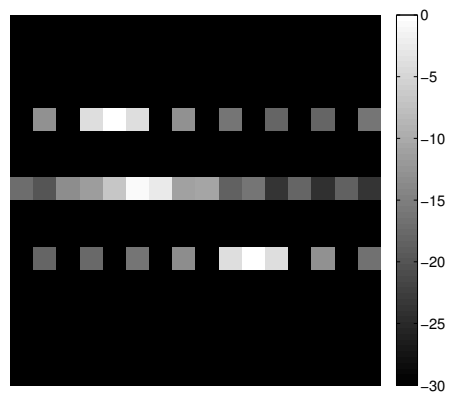

(b)

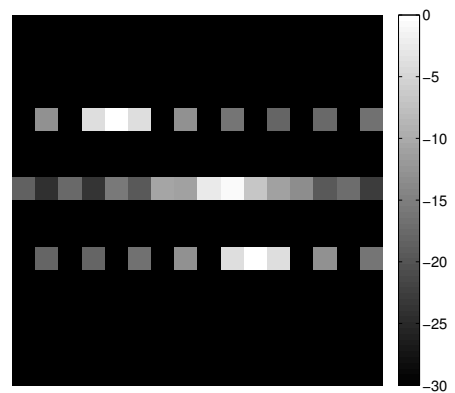

(c)

Figure 2.7: Subaperture images of a synthetic SAR scene containing two static targets and a moving target (in the scene center). (a) Conventional reconstruction (with quadratic phase errors due to a moving target). (b) First subaperture image. (c) Second subaperture image. 


\section{Chapter 3}

\section{Proposed Method for Moving Target Imaging}

This chapter provides a detailed formulation of our proposed method for moving target imaging with SAR. It begins with the construction of a subaperture matrix which is processed with low-rank and sparse decomposition to separate the moving targets from the static background. Subsequently, full-resolution images are reconstructed for the sparse and low-rank decomposed components. Further processing of the extracted moving targets is carried out by a sparsity-driven focusing method. Likewise, the background (low-rank) component is processed with a region-enhanced approach.

\subsection{Separation of Static and Dynamic Components}

Our proposed approach is primarily based on the LRSD of subaperture SAR data. It exploits the fact that moving targets show dynamic behavior in term of their spatial position in different subapertures. In contrast, static parts of the scene have consistent positions in all the subapertures. Figure 3.1 furnishes an overview of our approach in the form of a block diagram. Extraction of moving targets from the stationary background is an essential step for their proper imaging. This can be accomplished by LRSD of the complex SAR scene exploiting the SAR subapertures. A conventionally reconstructed complex SAR image $I \in \mathbb{C}^{n \times m}$ could be used to construct the subaperture images. Here we consider a band-limited Fourier transform as the forward model to reconstruct the complex SAR image from the raw data $r$. 


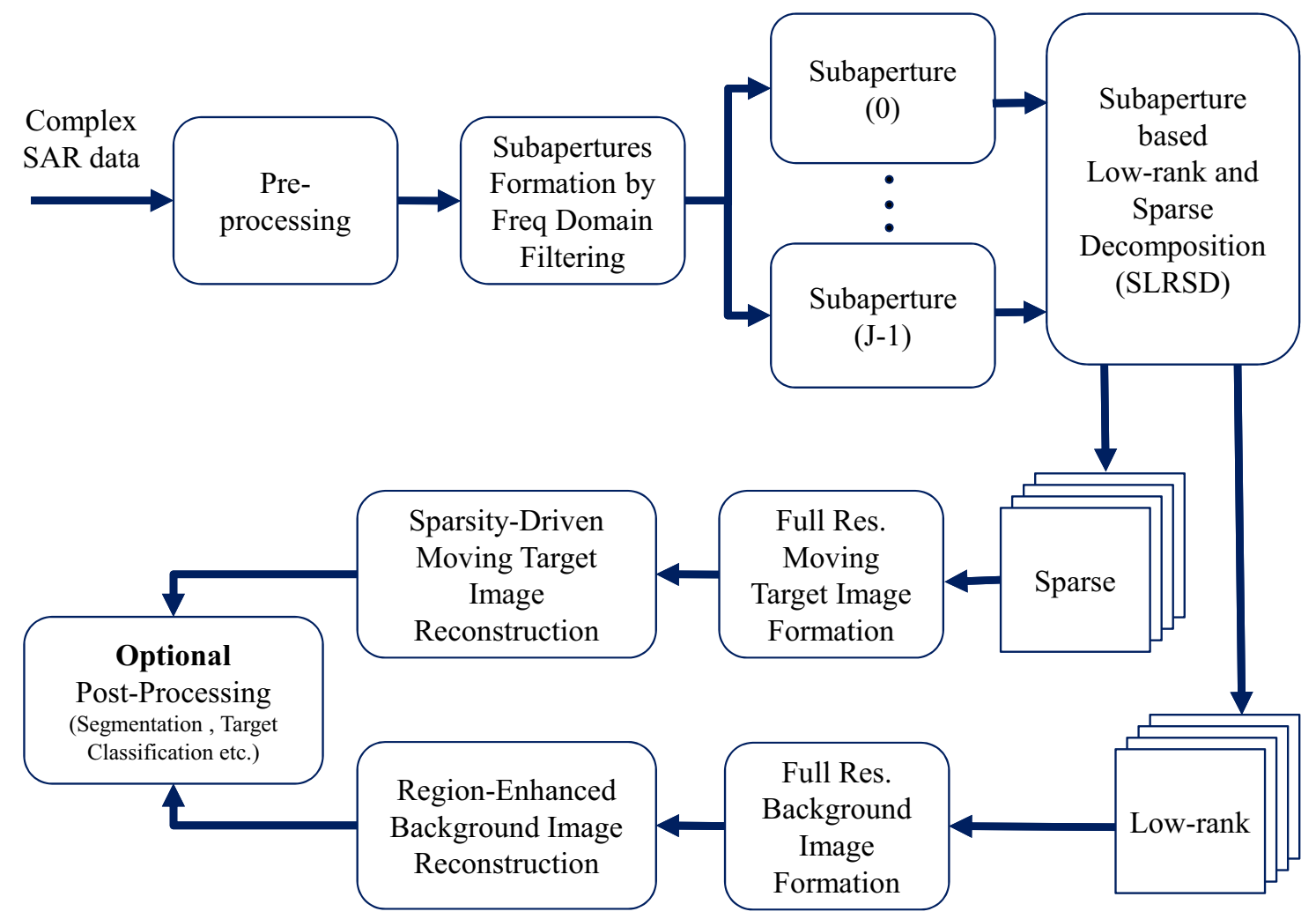

Figure 3.1: Block Diagram of the proposed moving target imaging approach. 


\subsection{Subapertures and the Data Matrix for LRSD}

In order to tailor the LRSD framework for the moving target imaging problem, multiple temporal images of the SAR scene should be stacked to form the matrix $D \in \mathbb{C}^{p \times J}$ as given below:

$$
D=\left[\begin{array}{ccccc}
\mid & \mid & & & \mid \\
d_{(0)} & d_{(1)} & \cdots & . & d_{(J-1)} \\
\mid & \mid & & & \mid
\end{array}\right]
$$

where each column in $D$ is a vectorized temporal image of the same SAR scene and we have $p=n \times m$. This is achieved by constructing $J$ subaperture SAR images and arranging them as columns of this matrix for LRSD. The first step is to apply the discrete Fourier transform (DFT) in azimuth to the complex SAR image $I_{(n, m)}$ containing unfocused moving targets. This is followed by a rectangular filtering operation where non-overlapping filtering windows are used to extract the frequency domain subapertures. This frequency domain filtering process can be described as follows:

$$
\begin{aligned}
& \Omega_{(k, m, i)}=\left(\sum_{n=0}^{N-1} I_{(n, m)} \cdot e^{-j 2 \pi k n / N}\right) \times \operatorname{rect}\left(\frac{k-i L}{L}\right) \\
& \text { where } n, k \in\{0, \ldots, N-1\}, \quad m \in\{0, \ldots, M-1\}
\end{aligned}
$$

where $i$ is the subaperture number, $n$ is the azimuth sample number, $k$ is the frequency bin number, and $m$ is the range bin number. Moreover, $L$ is the length of a subaperture window, i.e., the number of nonzero elements in the rectangular window function. Each subaperture image is then reconstructed by a zeropadded inverse discrete Fourier transform (IDFT) of original image size as given below:

$$
d_{(n, m, i)}=\frac{1}{N}\left(\sum_{k=0}^{N-1} \Omega_{(k, m, i)} \cdot e^{j 2 \pi k n / N}\right)
$$

The column vectors for the matrix $D$ are constructed from the subaperture images as follows:

$$
d_{i}=\left[d_{(0,0, i)}, d_{(1,0, i)}, \ldots, d_{(N-1, M-1, i)}\right]^{T}
$$

The bandwidth of the azimuth signal is reduced in the process of subapertures formation and consequently the original azimuth resolution is degraded by a factor of $N / L$ as 
follows:

$$
\rho_{s}=\rho_{a} \times\left(\frac{N}{L}\right)
$$

where $\rho_{s}$ is the subaperture resolution and $\rho_{a}$ is the original azimuth resolution of the SAR image. However, this loss of resolution is compensated after the LRSD by combining the spectral information of all the subapertures followed by an IDFT. The recovery of original full-resolution is a prominent feature of our proposed framework.

\subsection{LRSD based on Subaperture Data (SLRSD)}

Next we turn to the low-rank and sparse decomposition part. A successful LRSD in this scenario is based on the fact that the static parts of the scene do not change position across the subapertures, whereas the moving targets shift their positions in every subaperture. An LRSD framework for SAR imaging was proposed in [28]. It exploits the spatially low-rank and sparse structure of a stationary scene by making overlapping patches of the SAR image. This LRSD approach produces good results for a SAR scene with a few sparse objects (may not to be moving) and a slowly varying patch-wise low-rank background. However, it is not well suited for a scene that contains moving targets and where the background is not necessarily spatially low-rank. Here we extend the LRSD framework for the case of moving targets. Instead of making a patch based data matrix, we form a subaperture-based matrix as described above to exploit

the sparse nature of the moving targets. In this case, the background is not strictly required to be spatially low-rank as we enforce low-rank across the subapertures where typically fewer changes are induced by the static components over multiple subaperture data.

One can reasonably assume that the composite subaperture data comprises of the sparse component $S$, the low-rank component $B$ and the observation noise $n$. It leads to the following observation model:

$$
g=H \Theta R^{*}(B+S)+n
$$

where $\Theta$ is the random phase of the reflectivity field and $H$ is the forward model which 
takes into account the imaging process. A band-limited Fourier transform was assumed for this purpose. $R^{*}$ is a matrix reshaping operator which arranges the data in a column vector format. In (3.6), it should be noted that the composite subaperture data $g$ lies in the phase history domain and it is related to our subaperture data matrix $D$ as $g \approx H D$. Moreover, the LRSD assumes that $D$ comprises of low-rank component $B$ and sparse component $S$. The objective of LRSD in this case is to optimize for $S, B$ and $\Theta$. The overall optimization problem is formulated as follows:

$$
\begin{gathered}
\arg \min _{S, B, \Theta}\left\|g-H \Theta R^{*}(B+S)\right\|_{2}^{2}+\lambda_{b}\|B\|_{*}+\lambda_{s}\|S\|_{1} \\
\text { s.t. } \quad\left|\Theta_{(i, i, i)}\right|=1 \quad \forall i
\end{gathered}
$$

It should be noted that in our case both $S$ and $B$ consist of multiple subaperture images corresponding to the sparse and low-rank components respectively. $\lambda_{b}$ and $\lambda_{s}$ are the regularization parameters that balance the sparsity and low-rank conditions. The first term in this problem enforces data fidelity which defines how closely the final solution relates to the observed data. In other words, it dictates the reliability of our observation process. The solution of the LRSD problem in the presence of data fidelity term is facilitated by the introduction of an auxiliary variable $W$ as follows:

$$
\begin{array}{r}
\arg \min _{W, S, B, \Theta}\left\|g-H \Theta R^{*}(W)\right\|_{2}^{2}+\lambda_{b}\|B\|_{*}+\lambda_{s}\|S\|_{1} \\
\text { s.t. } W=B+S, \quad\left|\Theta_{i, i, i}\right|=1 \quad \forall i
\end{array}
$$

\subsection{Solving the Lagrangian Form of SLRSD}

In order to solve the LRSD optimization problem, we follow a similar approach to [28]. By including the major constraint as a penalty term in the overall cost function, the augmented Lagrangian form of (3.8) can be developed as follows:

$$
\begin{gathered}
L(W, B, S, \Theta, \Gamma)=\arg \min \left\|g-H \Theta R^{*}(W)\right\|_{2}^{2}+\lambda_{b}\|B\|_{*} \\
+\lambda_{s}\|S\|_{1}+\langle\Gamma, W-B-S\rangle+\frac{\beta}{2}\|W-B-S\|_{F}^{2} \\
\text { s.t. }\left|\Theta_{(i, i, i)}\right|=1 \quad \forall i
\end{gathered}
$$


The objective is to solve for $W, B, S, \Theta$ and $\Gamma$. Where $\Gamma$ is the Lagrange multiplier and $\beta$ is a positive constant that enforces the $W=B+S$ constraint. The overall optimization problem is solved iteratively in a step-wise manner. Using the alternating direction method of multipliers (ADMM), at each step the problem is solved for a single variable while all other variables are kept constant. Soft thresholding is used to solve for the sparse component $S$. Solution for the background (low-rank) $B$ component is obtained by a singular value thresholding (SVT) operation based on the singular value decomposition (SVD) of the data. The subproblem for the phase matrix $\Theta$ is solved by a fixed point algorithm as described in [14]. This process is repeated at each iteration until convergence is achieved or the stopping criterion is satisfied. In particular, each subproblem and its solution is described in the following subsections.

\subsubsection{Update of the Sparse Component $S$}

The solution for the sparse matrix involves soft thresholding. In order to solve for $S$, all other variables are held constant which leads to the following subproblem:

$$
\arg \min _{S} \lambda_{s}\|S\|_{1}+\langle\Gamma, W-B-S\rangle+\frac{\beta}{2}\|W-B-S\|_{F}^{2}
$$

Where the constant terms were dropped. This subproblem has a form which is very

similar to the least absolute shrinkage and selection operator (LASSO) problem [51] and it is solved by soft thresholding. The soft thresholding operator is defined as:

$$
\widetilde{T}_{\epsilon}(S)= \begin{cases}S-\epsilon & \text { if } \quad S>\epsilon \\ S+\epsilon & \text { if } \quad S<-\epsilon \\ 0 & \text { otherwise }\end{cases}
$$

Consequently, the solution to this subproblem is given by:

$$
S=\widetilde{T}_{\frac{\lambda_{s}}{\beta}}\left(W-B+\frac{\Gamma}{\beta}\right)
$$




\subsubsection{Update of the Background (Low-rank) Component $B$}

The low-rank component corresponding to the background image is updated in this step. Keeping other terms constant, the subproblem for $B$ is as follow:

$$
\arg \min _{B} \lambda_{b}\|B\|_{*}+\langle\Gamma, W-S-B\rangle+\frac{\beta}{2}\|W-S-B\|_{F}^{2}
$$

The solution to this problem requires nuclear norm minimization which is achieved by singular value thresholding (SVT). Therefore, this subproblem has the following solution:

$$
B=U \widetilde{T}_{\frac{\lambda_{b}}{\beta}}(\Sigma) V^{T}
$$

where the singular value decomposition of the matrix $\left(W-S+\frac{\Gamma}{\beta}\right)$ is given by $\left(U \Sigma V^{T}\right)$. The thresholding is an element-wise operation so it is computed efficiently. However, the major operation in this step is the singular value decomposition (SVD).

\subsubsection{Update of the Composite Matrix $W$}

Here, we update the composite matrix $W$. With other terms being constant, the subproblem for this step is given by:

$$
\arg \min _{W}\left\|g-H \Theta R^{*}(W)\right\|_{2}^{2}+\langle\Gamma, W-S-B\rangle+\frac{\beta}{2}\|W-S-B\|_{F}^{2}
$$

This is a quadratic optimization problem for which a closed form solution can be found as follows:

$$
\left(2\left(H \Theta R^{*}\right)^{H}\left(H \Theta R^{*}\right)+\beta I\right) W=\left(2\left(H \Theta R^{*}\right)^{H} g+\beta(B+S)-\Gamma\right)
$$

In practice, we solve this subproblem by few iterations of the conjugate gradient algorithm.

\subsubsection{Update of the Phase Matrix $\Theta$}

Solving for the unknown random phase involves defining a new vector $p$ containing the diagonal elements of phase matrix $\Theta$. The phase subproblem is defined as:

$$
\widehat{p}=\arg \min _{p}\|g-H \widetilde{X} p\|_{2}^{2}+\lambda_{p} \sum_{i=1}^{N}\left(\left|p_{i}\right|-1\right)^{2}
$$


where $\tilde{X}$ is the diagonal matrix comprising the elements of $R^{*} W$. This subproblem is solved by a fixed point algorithm as described in [14].

\subsubsection{Update of the Lagrange Multiplier $\Gamma$ and $\beta$}

The Lagrange multiplier $\Gamma$ should be updated every iteration by a factor of $\beta$. This update can be defined as:

$$
\Gamma^{(k+1)}=\Gamma^{(k)}+\beta(W-B-S)
$$

Ideally, a large value of $\beta$ should be used but it leads to slow convergence. Thus, a gradual increment approach is used. Starting with a small value, $\beta$ is gradually increased at each iteration. The overall convergence criterion is defined as $\frac{\left\|\left.W\right|^{(k+1)}-|W|^{(k)}\right\|_{F}}{\left\||W|^{(k)}\right\|_{F}}<\delta_{w}$.

\subsection{Full-resolution Reconstruction after LRSD}

After a successful decomposition of background and sparse components, we reconstruct full-resolution images for both components. Since we initially generated nonoverlapping subapertures, reconstruction of full aperture sparse and low-rank images is conveniently accomplished by a sum operation in the frequency domain. In particular, we combine the spectral information of the sparse decomposed subapertures as follows:

$$
\widetilde{S}_{(k, m)}=\widetilde{S}_{(k, m, 0)}+\widetilde{S}_{(k, m, 1)}+\ldots+\widetilde{S}_{(k, m, J-1)}
$$

Where $\widetilde{S}_{(k, m)}$ is the frequency domain full-resolution sparse component, $\widetilde{S}_{(k, m, i)}$ are the sparse subapertures and $J$ denotes the total number of subapertures. A full-resolution sparse image containing the moving target phase histories and reflectivity profiles can be reconstructed by an IDFT operation:

$$
S_{(n, m)}=\frac{1}{N}\left(\sum_{k=0}^{N-1} \widetilde{S}_{(k, m)} \cdot e^{j 2 \pi k n / N}\right)
$$

Similarly, the full-resolution background component in frequency domain $\widetilde{B}_{(k, m)}$ is obtained by:

$$
\widetilde{B}_{(k, m)}=\widetilde{B}_{(k, m, 0)}+\widetilde{B}_{(k, m, 1)}+\ldots+\widetilde{B}_{(k, m, J-1)}
$$


Algorithm 1 Proposed Algorithm for the SAR Moving Target Imaging

1. Inputs: Raw SAR data $(r)$, number of subapertures $J$, forward model $H$, regularization parameters: $\lambda_{b}, \lambda_{s}, \lambda_{p}$ and error tolerances: $\delta_{w}, \delta_{p}$

\section{Subaperture Matrix $D$ Construction:}

Use frequency domain filtering to construct subaperture matrix $\mathrm{D}$ from raw data $r$ : $D=\left[\begin{array}{llll}d_{\text {Subapr }(0)} & d_{\text {Subapr }(1)} & \ldots & d_{\text {Subapr }(J-1)}\end{array}\right]$ where $d_{\text {Subapr }(i)}$ are column vectors.

3. Initialization and LRSD: $W^{(0)}=|D|, p^{(0)}=e^{j \phi(D)}, S^{(0)}=0, B^{(0)}=W^{(0)}$, $\Gamma^{(0)}=0$, iteration counter $k=0$

while stopping criteria $\frac{\left\||W|^{(k+1)}-|W|^{(k)}\right\|_{F}}{\left\||W|^{(k)}\right\|_{F}}<\delta_{w}$ is not satisfied do

(a) Sparse Matrix $S$ Update:

Perform soft thresholding, $S=\widetilde{T}_{\frac{\lambda_{s}}{\beta}}\left(W-B+\frac{\Gamma}{\beta}\right)$

(b) Low-rank Matrix $B$ Update:

(i) Perform singular value decomposition $\left(U \Sigma V^{T}\right)$ of $\left(W-S+\frac{\Gamma}{\beta}\right)$

(ii) Perform singular value thresholding (SVT): $B=U \widetilde{T}_{\frac{\lambda_{b}}{\beta}}(\Sigma) V^{T}$

(c) Phase Matrix $\Theta$ Update:

Update $\Theta$ by solving (3.17) until $\frac{\left\|p^{(n+1)}-p^{(n)}\right\|}{\left\|p^{(n)}\right\|}<\delta_{p}$

(d) Composite Matrix $W$ Update:

Using conjugate gradient algorithm, solve (3.15) and update $W$

(e) Lagrangian Multiplier $\Gamma$ Update:

$\Gamma^{(k+1)}=\Gamma^{(k)}+\beta(W-B-S)$

Gradually increase $\beta:(\beta=\xi \beta, \xi>1)$

$k=k+1$

end while

\section{Full Resolution Reconstruction:}

(i) Combine the decomposed subapertures in the frequency domain:

$\widetilde{S}=\widetilde{S}_{\operatorname{Subapr}(0)}+\widetilde{S}_{\text {Subapr }(1)}+\ldots+\widetilde{S}_{\text {Subapr }(J-1)}$

$\widetilde{B}=\widetilde{B}_{\operatorname{Subapr}(0)}+\widetilde{B}_{\operatorname{Subapr}(1)}+\ldots+\widetilde{B}_{\operatorname{Subapr}(J-1)}$

(ii) Perform IDFT to reconstruct full-resolution images of $S$ and $B$.

\section{Further Processing:}

(i) Process $S$ (moving targets) with sparsity-driven focusing (SDF).

(ii) Process $B$ (SAR background) with region-enhanced SAR imaging. 
and the full-resolution background image is reconstructed with another IDFT step:

$$
B_{(n, m)}=\frac{1}{N}\left(\sum_{k=0}^{N-1} \widetilde{B}_{(k, m)} \cdot e^{j 2 \pi k n / N}\right)
$$

Finally, we reconstruct the moving target image with the sparsity-driven focusing. Nevertheless, any other moving target imaging algorithm could also be used. Similarly, the background image may be reconstructed with the region-enhanced SAR imaging method [13] which suppresses the irregularities and produces a smooth SAR background. Algorithm 1 summarizes the proposed method. 


\section{Chapter 4}

\section{Experimental Results}

This chapter provides the details of our experimental results with synthetic and real SAR backgrounds. A comprehensive performance analysis of the proposed method using numerical simulations is also included.

\subsection{Experimental Results with Synthetic Targets}

We have performed several experiments to validate the performance of our proposed method. Most of these experiments used two subapertures to make the simulation results more convenient for demonstration and to limit the number of generated figures. Nevertheless, we have studied the impact of different number of subapertures later in this chapter. In this set of experiments, we have added synthetic moving targets to the SAR scene with a low SCR. All images are presented on a logarithmic scale for a better dynamic range.

\subsubsection{Synthetic Scene Experiments}

First, we performed some experiments on fully synthetic scenes where we simulated the background as well as the moving targets. We constructed a random background and simulated the moving targets by modulating a quadratic phase error in the azimuth direction. Figure 4.1 reveals the results of this experiment. Three moving targets were placed near the scene center with a very low SCR of $-3.2 \mathrm{~dB}$ as shown in Figure 4.1(a). The target motion leads to the spreading of their response along the direction 


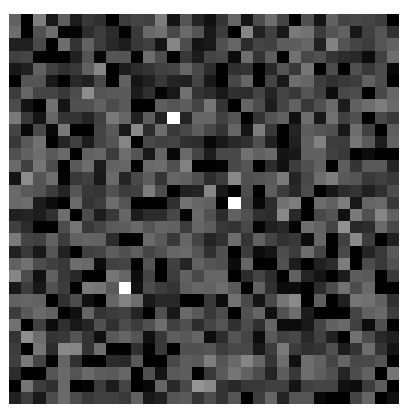

(a)

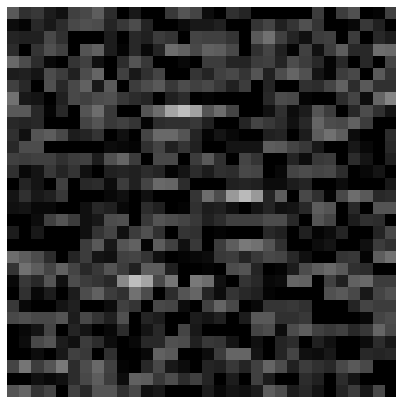

(d)

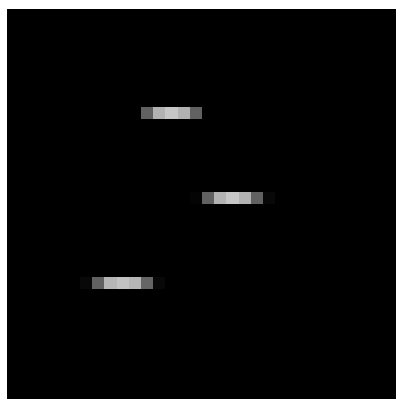

(g)

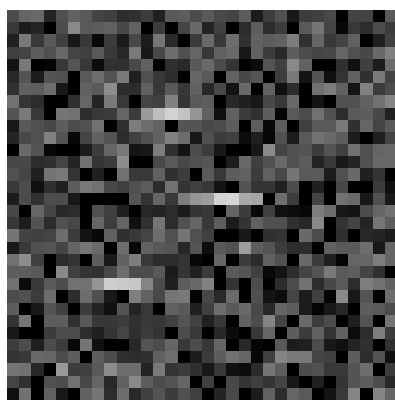

(b)

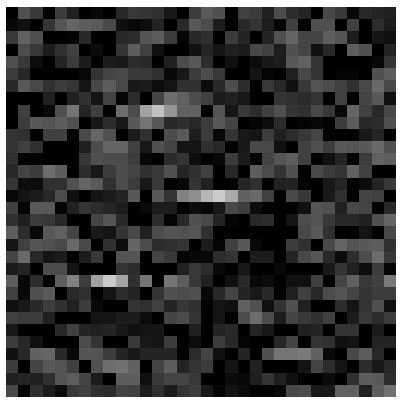

(e)

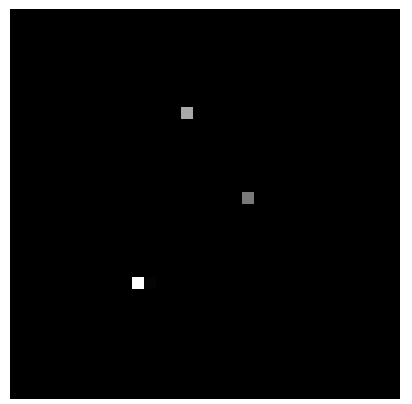

(h)

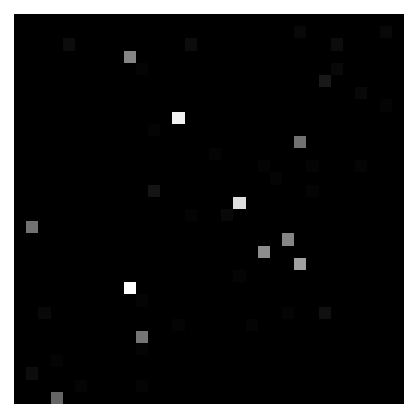

(c)

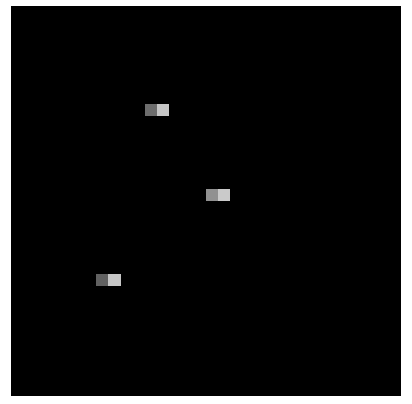

(f)

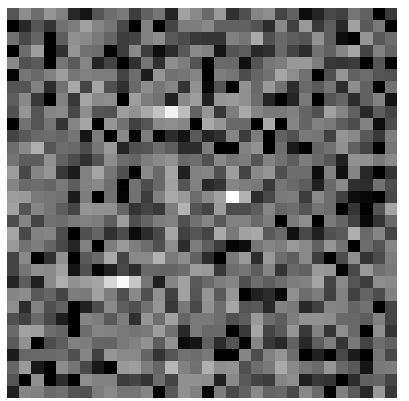

(i)

Figure 4.1: Results of the synthetic scene experiment. (a) Ground truth scene indicating the locations of three moving targets in a noisy background and low SCR. (b) Conventionally reconstructed scene with quadratic phase errors due to the targets' motions. (c) Focused image produced by the SDF algorithm without LRSD (d) First subaperture image with a reduced azimuth resolution. (e) Second subaperture image with a reduced azimuth resolution. (f) Sparse image after LRSD, corresponding to the first subaperture. (g) Full resolution conventional image of the sparse part containing the moving targets. (h) Focused moving target image after LRSD and sparsity-driven focusing. (i) Background image after LRSD and the full-resolution conventional reconstruction. 
of motion in the conventional reconstruction, as shown in Figure 4.1(b). Figure 4.1(c) demonstrates the output of the sparsity-driven focusing [17] without LRSD. There were eight spurious points in addition to the three original moving targets. It follows that the direct application of sparsity-driven focusing could not perform well under very low SCR conditions and the LRSD step becomes imperative.

Two subapertures were constructed using square window functions for the frequency domain filtering to mask half of the azimuth spectrum at a time followed by a zeropadded IDFT. Figure 4.1(d, e) depict the subaperture images formed this way. The resolution along the horizontal axis (azimuth) was lowered due to the masked frequency samples. This loss of resolution is unavoidable at this stage since subaperture images are required for the LRSD step, but it is recovered later.

A slight shift of the mean position of the moving targets may be noticed along the azimuth axis which is necessary for the LRSD to detect the moving targets as sparse components. Our formulation in (3.8) leads to the LRSD of the scene as well as the corresponding data through subaperture-based processing. Then one can process

the full aperture data for the sparse and low-rank components separately in various ways. Just as an example, here we demonstrate the use of [17] to process the data corresponding to the sparse component which contains the moving targets. As shown in Figure 4.1(h), the image is well focused without any ghost targets. Two subaperture low-rank images were also obtained from the LRSD (not shown). These images were conventionally reconstructed to get the full-resolution background image as shown in Figure 4.1(i).

\subsubsection{Experiments with Real SAR Background}

Real SAR background experiments were performed with the MiniSAR data [52] where we have introduced synthetic moving targets for performance evaluation. Figure 4.2 depicts the results of our real SAR scene experiments. Three moving targets were added to a $64 \times 64$ real SAR background where one of the targets has a bigger signature as compared to the other two. Furthermore, four static targets were added in the scene to evaluate the LRSD performance for the strong static components. Figure 4.2(a, b) 


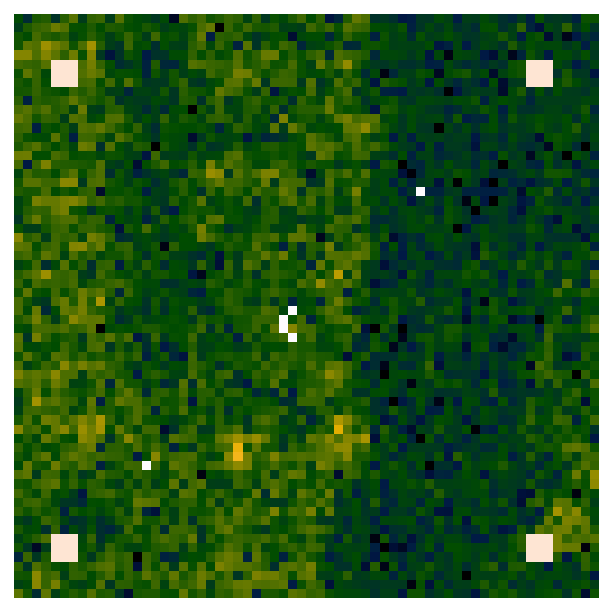

(a)

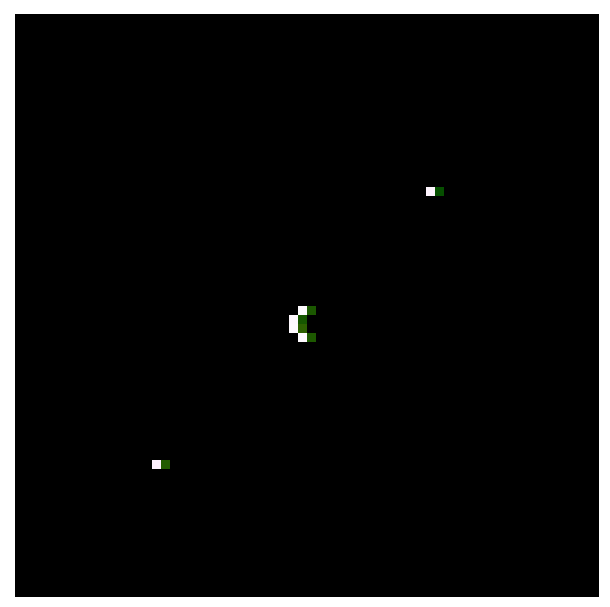

(c)

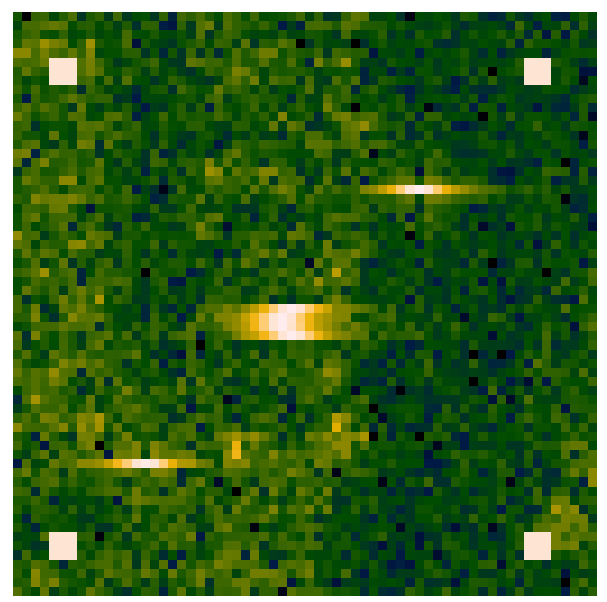

(b)

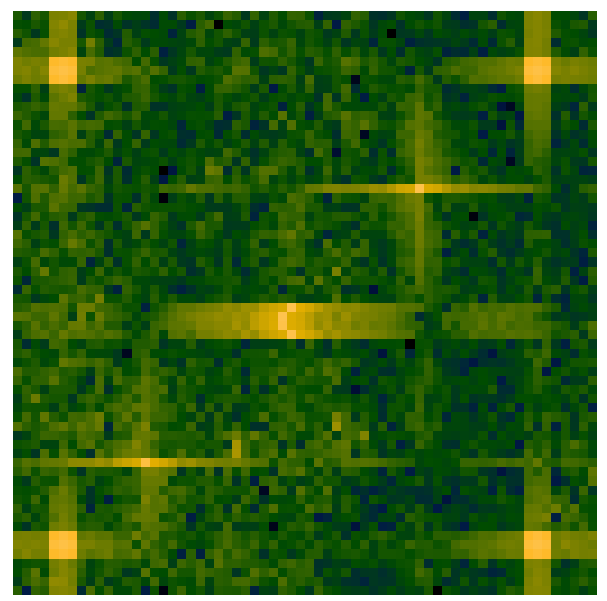

(d)

Figure 4.2: Results of the real SAR scene experiment. (a) Ground truth scene indicating the locations of three moving targets in a real SAR background. Four strong static points were also added near the corners of the scene. (b) Conventionally reconstructed scene with quadratic phase errors due to the targets' motions. (c) Focused moving target image after processing by the proposed framework and subsequent sparsity-driven focusing. (d) Background image after LRSD and full-resolution conventional reconstruction.

present the ground truth and the conventional reconstructions respectively.

Figure 4.2(c) shows the moving target image obtained after processing by our proposed framework and subsequent sparsity-driven focusing. It contains the focused mov- 
ing targets without any significant background or false targets.

The full-resolution background image is shown in Figure 4.2(d). It is worth noting that most of the MiniSAR scene is included in the low-rank (background) image since its components are mostly consistent across the subapertures. The four synthetic static targets also appear in the low-rank image for the same reason. Another important effect which requires attention is the leakage of the residual from the moving targets response. There are two main factors that cause this leakage. The first factor is that the moving target response (spread function) has some overlapping components across the subapertures which do not change significantly. The second factor is the regularization parameter values used for the LRSD. If we optimize for a sparser solution, some parts of the moving target response would be forced to the low-rank part.

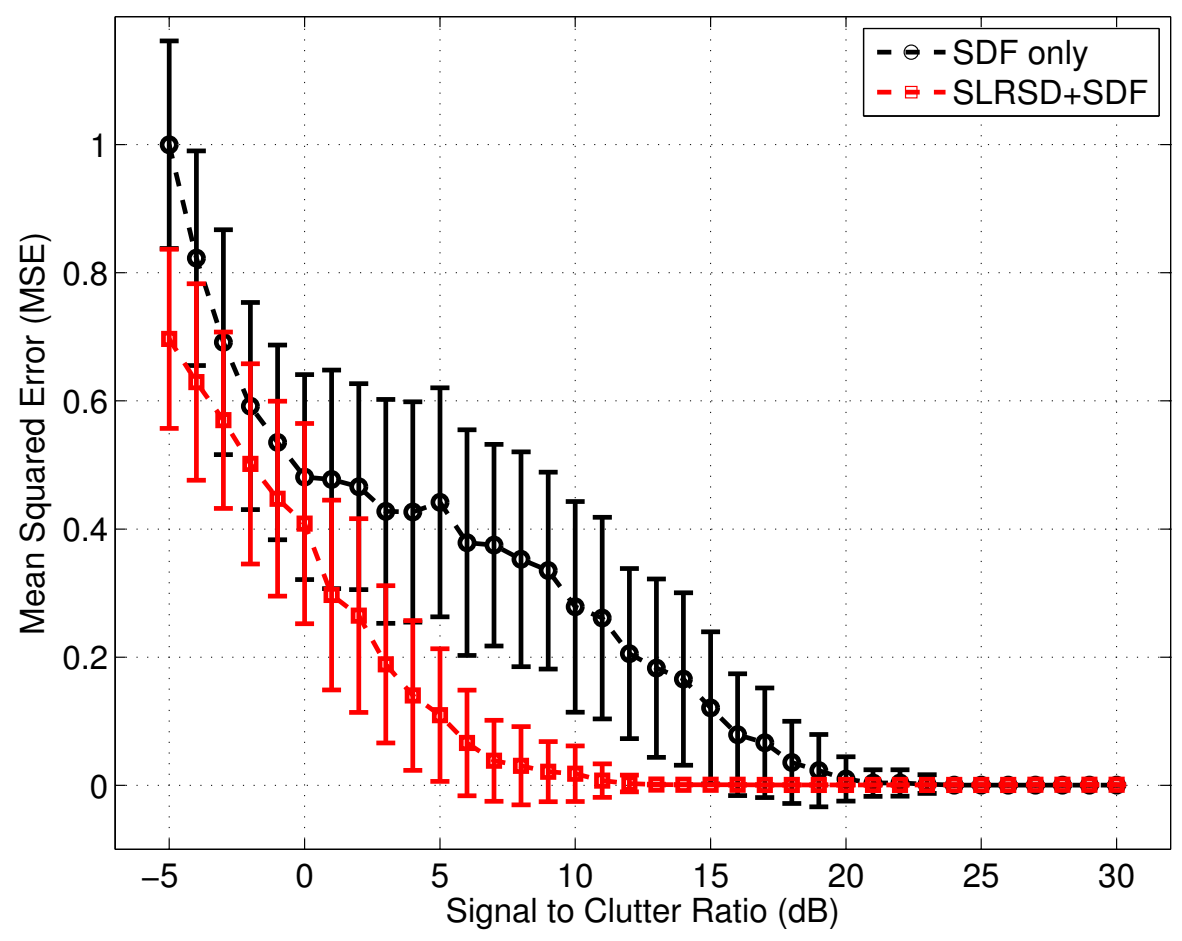

Figure 4.3: Signal to clutter ratio (SCR) vs. mean squared error (MSE) performance plots using the proposed approach (SLRSD+SDF, red) and the sparsity-driven focusing only (SDF, black). The standard deviation of each measurement is given by an associated error bar. 


\subsection{Numerical Simulations for Performance Evalu- ation}

We have performed a series of numerical simulations to further investigate the performance of our proposed approach. In particular, we have conducted experiments for the signal to clutter ratio (SCR) performance, impact of varying the number of subapertures and performance with different moving target velocities. Details of these experiments and their associated results are described in the following subsections.

\subsubsection{SCR Performance Analysis}

In these simulations, the objective was to examine the performance for a broad range of SCR scenarios. Particularly, we varied SCR in the range of $-5 \mathrm{~dB}$ to $+30 \mathrm{~dB}$ with a step size of $1 \mathrm{~dB}$. We have used a small synthetic SAR scene $(16 \times 16)$ with three moving targets (overall moving targets' arrangement was similar to Figure 4.1) where different SCR scenarios were simulated by varying the standard deviation of the random background while keeping the targets' amplitudes fixed. Mean squared error (MSE) and structural similarity index (SSIM) were used as the performance measures. SSIM is a measure of image reconstruction quality which provides a numerical value for

the extent of degradation of structural information [53]. The ground truth image was used as a reference for MSE and SSIM calculations. For each SCR value, the complete simulation was repeated 200 times and all corresponding results were saved.

The first set of experiments $(\mathrm{SCR}=-5 \mathrm{~dB}$ to $+30 \mathrm{~dB}, 200$ repetitions at each $\mathrm{SCR}$ value) used only the sparsity-driven focusing (SDF) to benchmark its SCR performance. In the second set of experiments, the data were processed with our proposed approach (SLRSD+SDF). The mean squared error plots for this experiment are provided in Figure 4.3 where the black curve indicates the SDF only results and the red curve corresponds to the SLRSD+SDF results. Each data point in these curves represents the mean of 200 MSE results for a particular SCR value whereas the vertical bars indicate the associated standard deviations. As a general trend, the standard deviation of the results decreases as the SCR increases. 
The SDF curve converges to zero mean squared error for an SCR value of approximately $21 \mathrm{~dB}$ whereas our proposed method achieves a similar performance for an SCR of $11 \mathrm{~dB}$ only. There is an improvement of approximately $10 \mathrm{~dB}$ in the SCR performance which might be very useful for the low-SCR moving target imaging scenarios. A similar trend was observed for the SSIM measure. The SSIM results are presented in Figure 4.4 where the means of 200 SSIM values for various SCR values are plotted. The improved performance of our proposed approach is evident as the SSIM value reaches its maximum (one) for an SCR of about 11dB.

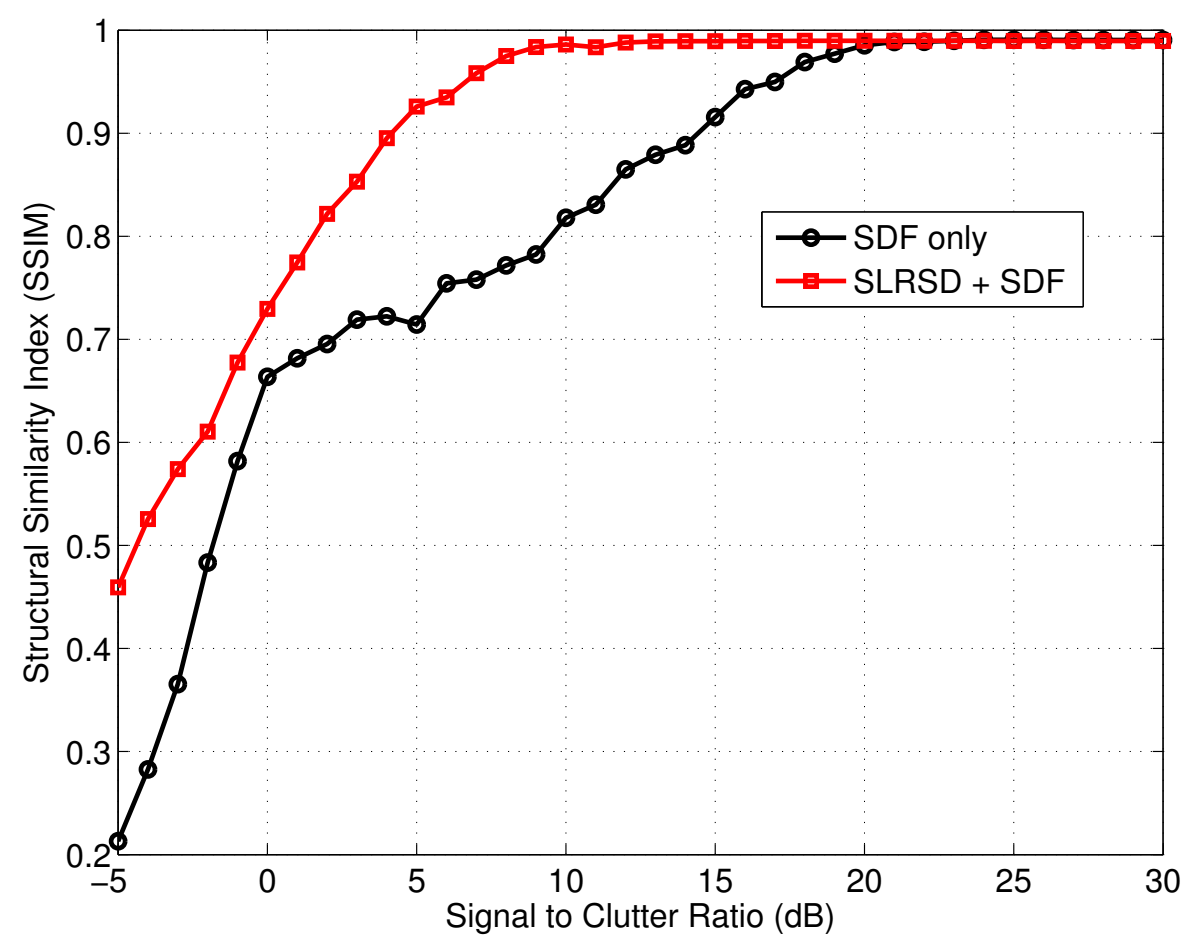

Figure 4.4: Signal to clutter ratio (SCR) vs. structural similarity index (SSIM) of the reconstructed SAR image for the proposed approach (red) and sparsity-driven focusing only (black).

\subsubsection{Target Velocity Variations}

In these experiments, we have introduced a single moving target (consisting of two point scatterers) and varied its azimuth velocity while keeping the other parameters fixed. The SAR system parameters used for these experiments are given in the Table 
4.1. The SCR was fixed to $15 \mathrm{~dB}$ and two subapertures were used in all runs. The total phase error induced due to a particular moving target velocity during the exposure time can be computed by the relation given in equation (2.9).

The proposed approach performed particularly well at very low target velocities. The SLRSD was able to resolve the moving target as a sparse component for very low phase errors. Here we present few representative results for some slow moving targets. Figure 4.5 illustrates the reconstruction results for moving target velocities of $1 \mathrm{~m} / \mathrm{s}$ and $3 \mathrm{~m} / \mathrm{s}$. Figure $4.5(\mathrm{a})$ presents the conventional reconstruction of a target moving at $1 \mathrm{~m} / \mathrm{s}$. The total phase error induced by this moving target was $0.5 \pi$ radians during an azimuth exposure time of $1 s(-0.5 s \leq \eta \leq+0.5 s)$. The full-resolution conventional image of the sparse component extracted by SLRSD is given in Figure 4.5(b) which highlights a successful decomposition of the sparse part containing the target's phase history despite a very low velocity. This sparse image was then focused with the SDF and presented in Figure 4.5(c). Similar convincing results were obtained for a target velocity of $3 \mathrm{~m} / \mathrm{s}$ (phase error $=1.5 \pi$ radians) as depicted by the second row of Figure 4.5 .

Table 4.1: System parameters used for the target velocity simulations.

\begin{tabular}{|l|l|}
\hline Parameter & Value \\
\hline Tx Frequency & $15 \mathrm{GHz}$ \\
\hline Wavelength, $\lambda$ & $0.02 \mathrm{~m}$ \\
\hline Resolutions, $\rho_{r}$ and $\rho_{a}$ & $1 \mathrm{~m}$ \\
\hline Range to the scene center, $R_{0}$ & $30 \mathrm{Km}$ \\
\hline Platform velocity, $V_{p}$ & $300 \mathrm{~m} / \mathrm{sec}$ \\
\hline Exposure Time, $T=\lambda R_{0} / 2 V_{p} \rho_{a}$ & $1 \mathrm{sec}$ \\
\hline
\end{tabular}




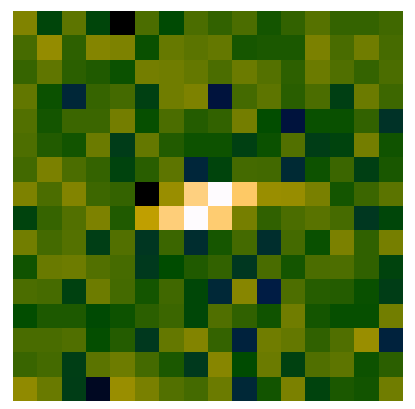

(a)

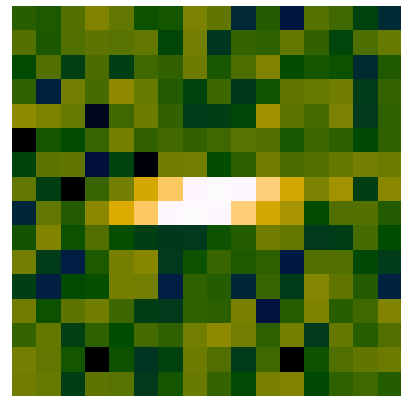

(d)

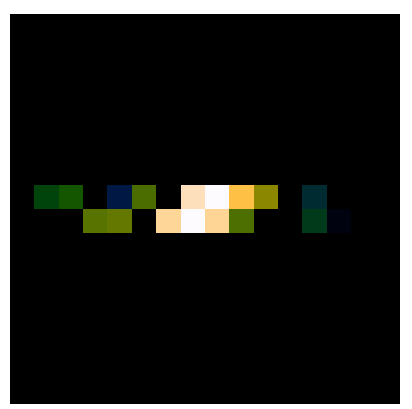

(b)

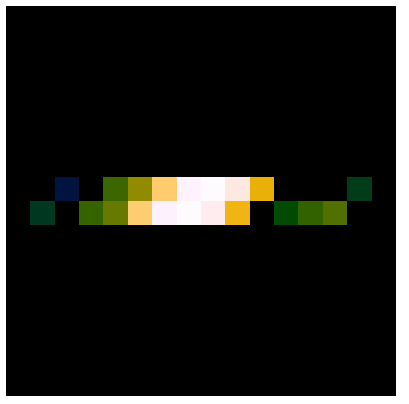

(e)

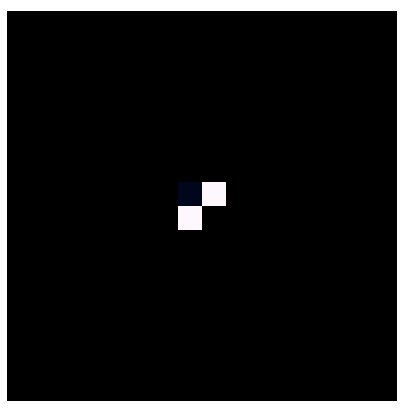

(c)

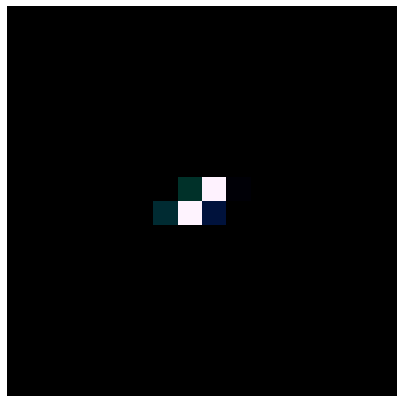

(f)

Figure 4.5: Velocity experiment results. (a) Conventional moving target image (Velocity $=1 \mathrm{~m} / \mathrm{s}$, Phase error $=0.5 \pi$ radians) (b) Full resolution conventional image of the sparse component extracted by SLRSD. (c) Moving target image after SLRSD+SDF. (d) Conventional moving target image (Velocity $=3 \mathrm{~m} / \mathrm{s}$, Phase error $=1.5 \pi$ radians). (e) Full resolution conventional image of the sparse component extracted by SLRSD. (f) Moving target image after SLRSD+SDF.

\subsubsection{Varying Number of Subapertures}

The number of subapertures is an important parameter which needs to be carefully selected since it is directly related to the azimuth resolution of the subaperture images used for the SLRSD. Here, we have analyzed the effect of various number of subapertures on the final image reconstruction quality. In this regard, we have followed an approach similar to the SCR performance experiments. In particular, on a $16 \times 16$ synthetic SAR image with three moving targets, we varied the SCR in a range of $+5 \mathrm{~dB}$ to $+20 \mathrm{~dB}$ with a $1 \mathrm{~dB}$ step size and saved the MSE values for 200 repetitions at each SCR step. The whole experiment was repeated four times once each for two, four, eight, and sixteen 
subapertures.

The MSE results were plotted after normalizing by the maximum MSE in Figure 4.6 for the 02,04 , and 08 subapertures. It is very evident that in comparison to 08 subapertures results, 02 and 04 subapertures performed much better. For a 16x16 image, eight subapertures degrade the azimuth resolution to such an extent that the moving targets are hardly decomposed as a sparse component. For the same reason, worst performance was observed for 16 subapertures (not shown due to scaling issues).

A magnified view of the MSE performance with 02 and 04 subapertures is presented in Figure 4.7 where the two curves indicate very close MSE values despite minute variations. The MSE plots are shown on a logarithmic scale in Figure 4.8. These simulation results suggest that a smaller number of subapertures leads to improved performance. As the number of subapertures approaches or exceeds half of the image dimension in azimuth (8 in this experiment), the performance drops considerably. Additional advantages of fewer subapertures are the reduced memory requirement and accordingly shorter processing time.

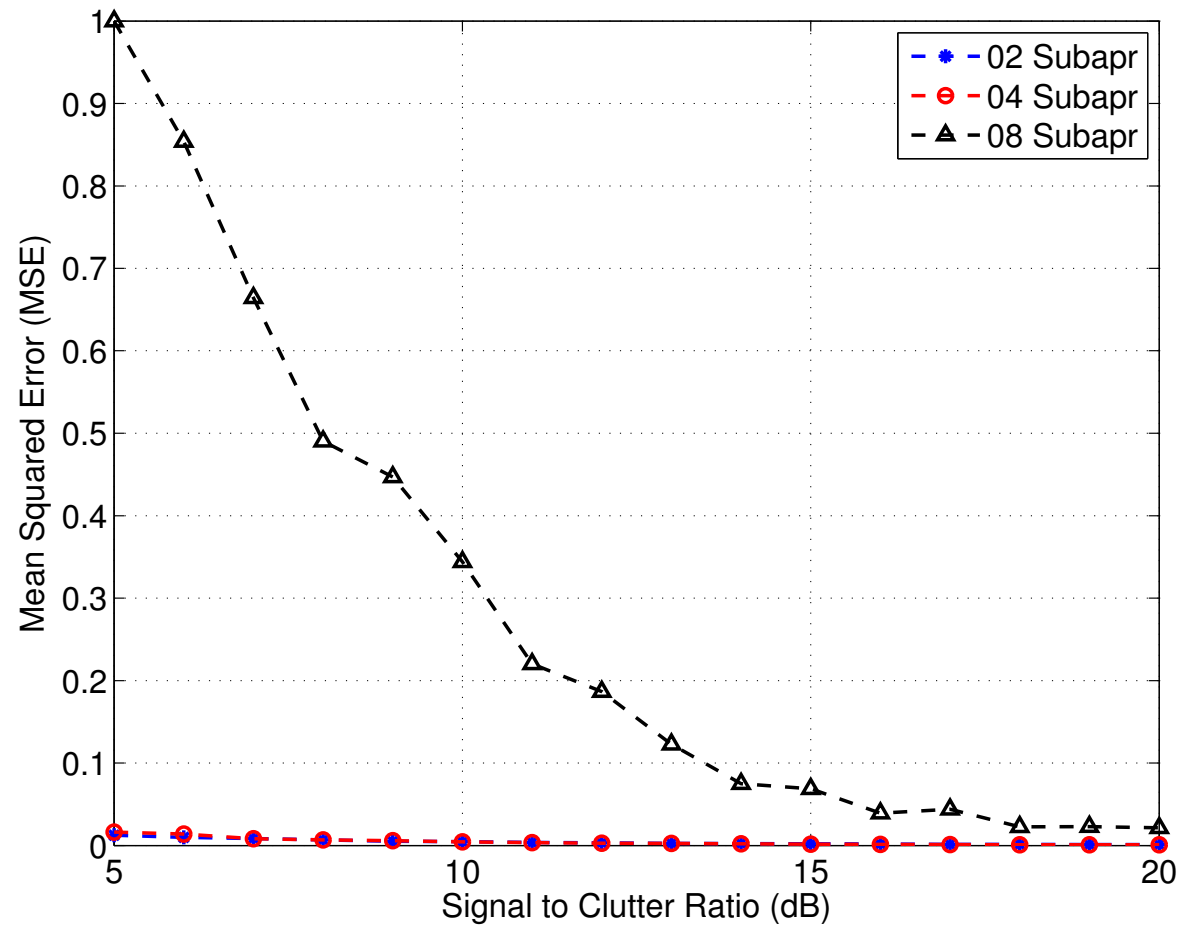

Figure 4.6: SCR vs. MSE plots for various number of subapertures (02, 04, 08). 


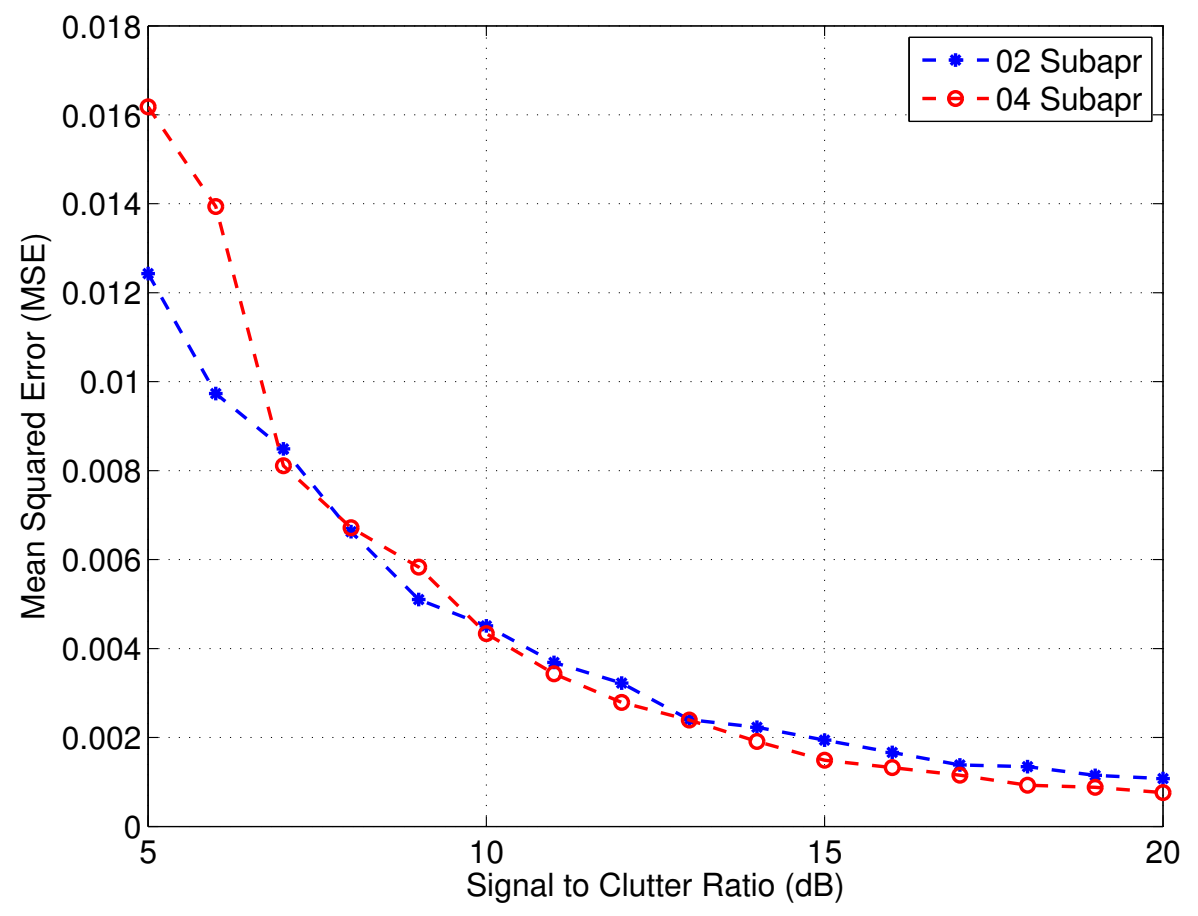

Figure 4.7: SCR vs. MSE plots for 02 and 04 subapertures.

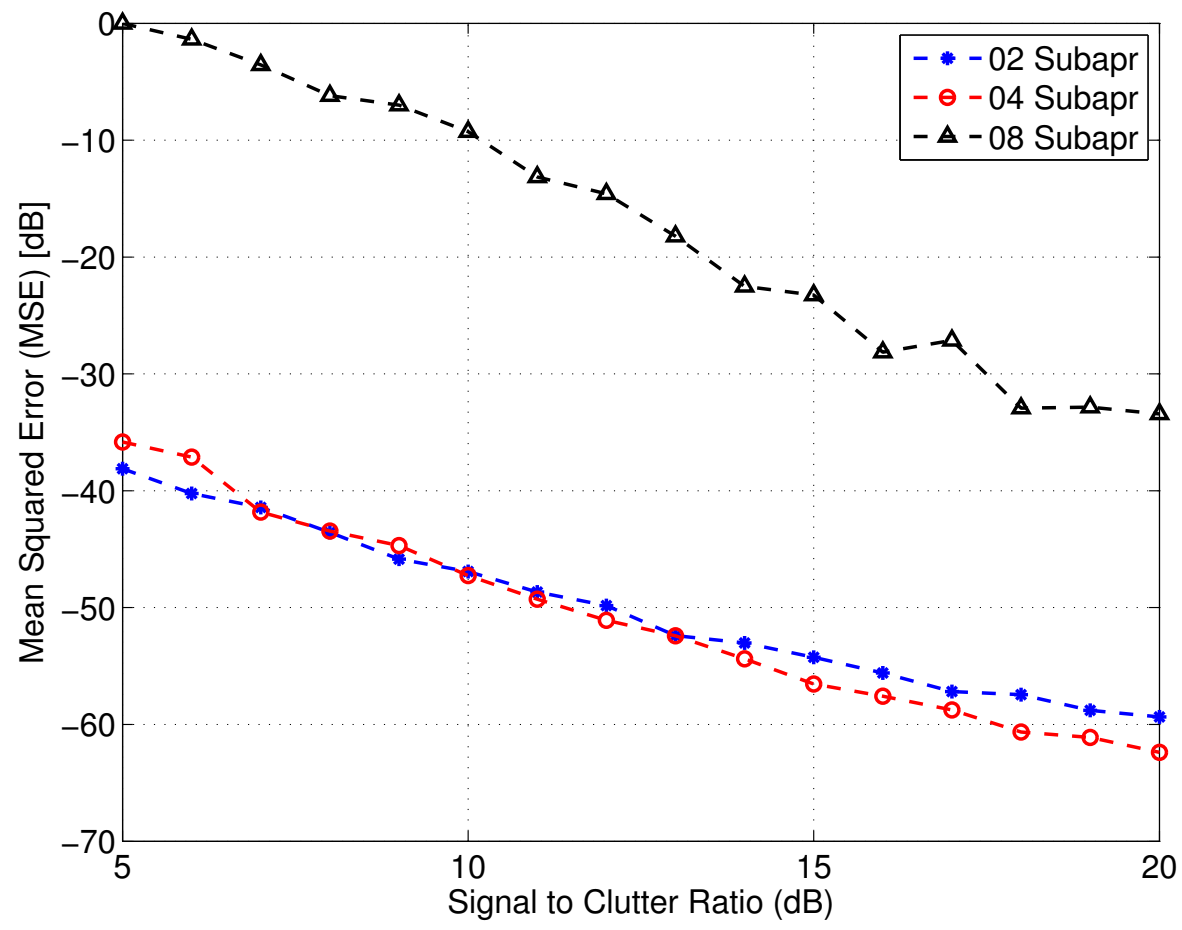

Figure 4.8: SCR vs. MSE plots for various number of subapertures $(02,04,08)$ on a logarithmic scale. 
Table 4.2: CPU specifications for SLRSD experiments

\begin{tabular}{|c|c|}
\hline Processor type & Intel Xeon E5620 \\
\hline CPU clock & $2.4 \mathrm{GHz}$ \\
\hline No. of processors & 02 \\
\hline Memory (RAM) & $96 \mathrm{~GB}$ \\
\hline System type & 64 -bit \\
\hline Operating system & Windows 7 Professional \\
\hline MATLAB version & R2014a \\
\hline
\end{tabular}

\subsection{Processing Time and Memory Usage}

For all of our experiments, we have used MATLAB version R2014a. The specifications of the CPU used for our experiments are given in Table 4.2. Our code was not optimized for memory usage and processing time since the main focus was to develop a functional code which implements our proposed algorithm. Further, we focused on numerical simulations and experiments with real SAR data. Code optimization for an improved CPU performance is a possible future work. Moreover, we discuss some possibilities of extending our framework to very large scenes in Chapter 6 . Table 4.3 presents the processing times and memory usage with the current version of our code and for various SAR image sizes.

Table 4.3: Processing time and memory usage

\begin{tabular}{|c|c|c|}
\hline SAR image size & SLRSD processing time & Memory usage \\
\hline $16 \times 16$ & $2.79 \mathrm{sec}$ & $315 \mathrm{~KB}$ \\
\hline $32 \times 32$ & $4.63 \mathrm{sec}$ & $1.18 \mathrm{MB}$ \\
\hline $100 \times 100$ & $8.49 \mathrm{sec}$ & $11.5 \mathrm{MB}$ \\
\hline $200 \times 200$ & $62.04 \mathrm{sec}$ & $46.1 \mathrm{MB}$ \\
\hline
\end{tabular}




\section{Chapter 5}

\section{Experiments with EMISAR Data}

This chapter describes the experiments with the Danish EMISAR data [54] containing actual moving targets. From a large SAR scene, various moving targets were selected and processed with our proposed framework and the corresponding results are presented. Overall, the performance of our subaperture based LRSD approach was very encouraging in these real SAR experiments.

\subsection{About EMISAR}

EMISAR was primarily developed for remote sensing applications. It is a fully polarimetric SAR ( $\mathrm{HH}, \mathrm{VH}, \mathrm{HV}$, and VV polarizations) operating in two frequency bands (L and C). Furthermore, it can perform SAR imaging with a $2 \mathrm{~m}$ resolution in the slant range and azimuth directions while operating at a flight altitude of 41,000 feet. The swath width at this resolution is around $12 \mathrm{~km}$. Detailed specifications of EMISAR are given in Table 5.1 .

The EMISAR data used for our experiments was acquired in C-band ( $5.3 \mathrm{GHz}$ ) from the water/bridge scene Storebaelt, Denmark. The data comprises of all polarizations, however we have used a single channel $(\mathrm{HH})$ for the moving target experiments. The complete SAR image is shown in Figure 5.1 which is colored by the various polarizations. The scene covers a large area most of which consists of sea. There are two land areas on the left and right extremes of the scene which are connected by a bridge. There are numerous boats and ships of various sizes moving in different directions in the sea. We 
have selected two regions of interest inside this large scene which are marked by the yellow boxes in Figure 5.1. In the following sections, we would describe the experimental results for different moving objects found in these regions.

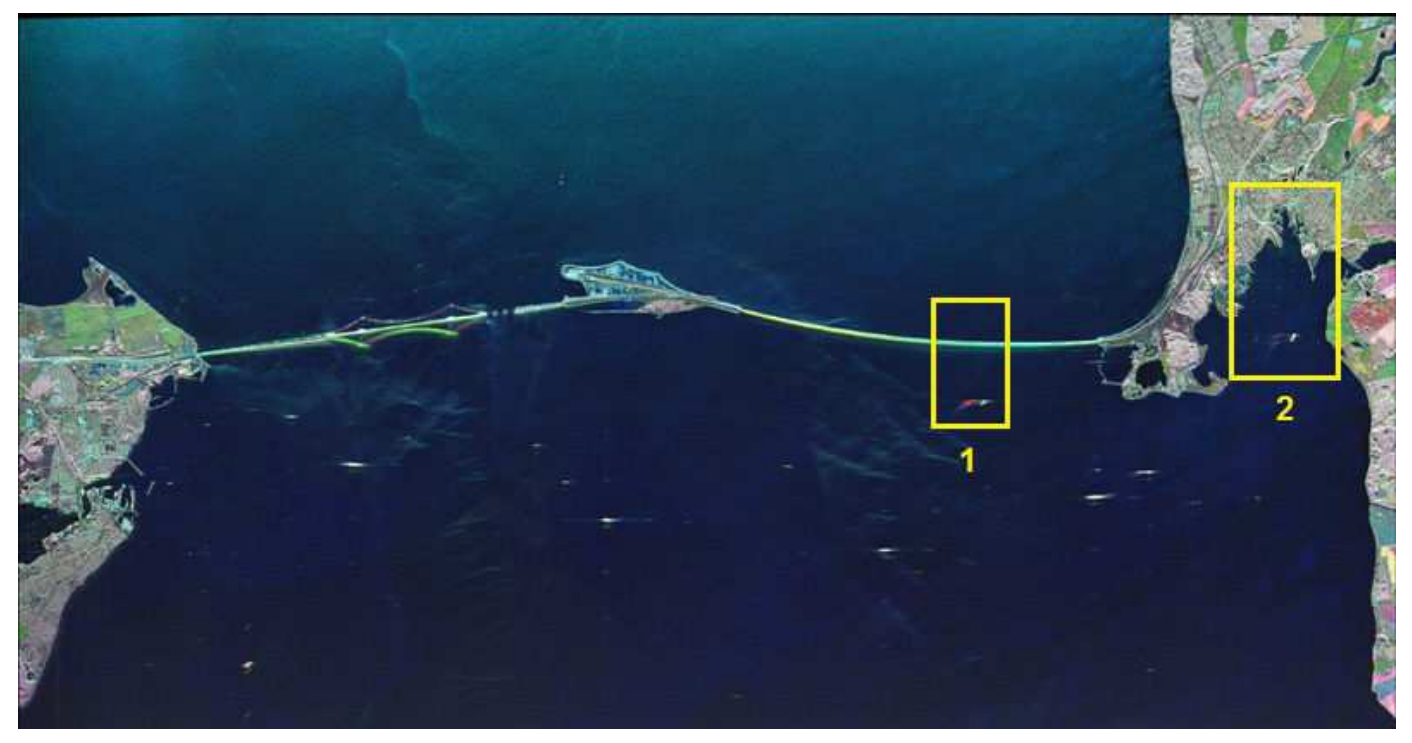

Figure 5.1: EMISAR water/bridge scene, Storebaelt, Denmark. Yellow boxes indicate some regions of interest for moving target imaging experiments. 
Table 5.1: EMISAR system parameters.

\begin{tabular}{|c|c|c|}
\hline System Parameters & C-Band specifications & L-Band specifications \\
\hline Frequency & $5.3 \mathrm{GHz}$ & $1.25 \mathrm{GHz}$ \\
\hline Output power & $2 \mathrm{~kW}$ & $6 \mathrm{~kW}$ \\
\hline Pulse length & $0.64-20 \mathrm{\mu s}$ & $0.64-20 \mathrm{\mu s}$ \\
\hline Max. bandwidth & $100 \mathrm{MHz}$ & $100 \mathrm{MHz}$ \\
\hline Antenna gain & $27 \mathrm{dBi}$ & $18 \mathrm{dBi}$ \\
\hline Azimuth beam width $(3 \mathrm{~dB})$ & $2.4^{\circ}$ & $10^{\circ}$ \\
\hline Elevation pattern width & $31^{\circ}$ & $42^{\circ}$ \\
\hline Polarization & Fully polarimetric & Fully polarimetric \\
\hline Resolution in slant range & 2,4 or $8 \mathrm{~m}$ & 2,4 or $8 \mathrm{~m}$ \\
\hline Resolution in azimuth & 2,4 or $8 \mathrm{~m}$ & 2,4 or $8 \mathrm{~m}$ \\
\hline Swath width & 12,24 or $48 \mathrm{~km}$ & 12,24 or $48 \mathrm{~km}$ \\
\hline Flight altitude & Typically $41,000 \mathrm{ft}$ & Typically $41,000 \mathrm{ft}$ \\
\hline
\end{tabular}




\subsection{Moving Target Scene 1 (region-1)}

A larger view of region-1 is given in Figure 5.2(a) where a relatively big moving target (ship) is visible close to the bridge. The target appears to have both range and azimuth velocity components since it is heading in a direction which is not parallel to the range or azimuth axis of the EMISAR. The cropped moving target part of the scene is depicted in Figure 5.2(b). On this scene, subaperture based low-rank and sparse decomposition was performed by making four subapertures. The composite subaperture images are presented in Figure 5.3, where a red line has been marked in the center of each image to emphasize the slight movement of the target in various subapertures. Figure 5.4 presents the four sparse subaperture images after the decomposition that contain mostly the moving target's reflectivity profile. Looking at the sparse subaperture images from bottom to top in Figure 5.4, movement of the target towards left is evident. We have deliberately stretched these images horizontally to pronounce the target's motion in subaperture images for better visual comprehension.

A full-resolution moving target image was reconstructed and it is shown in Figure 5.5(b). Similarly, the full-resolution background image is given in Figure 5.5(a). These results manifest that most parts of the moving target's reflectivity profile were extracted as the sparse component. However, some residual stays in the background image which corresponds to the low-rank portions of the overall moving target response.

We have processed the full-resolution background image with the region-enhanced SAR imaging algorithm [13] and the results are presented in Figure 5.5(c). Here, most non-uniformity is removed leading to a smooth image of the stationary background reflectivity. Any suitable type of SAR reconstruction algorithm may be used at this stage depending on the final application. The full-resolution moving target image was processed with SDF and the focused image is shown in Figure 5.5(d). Since it is a bigger target, it consists of multiple strong scatterers which were focused by the SDF and they are more prominent in the output image. 


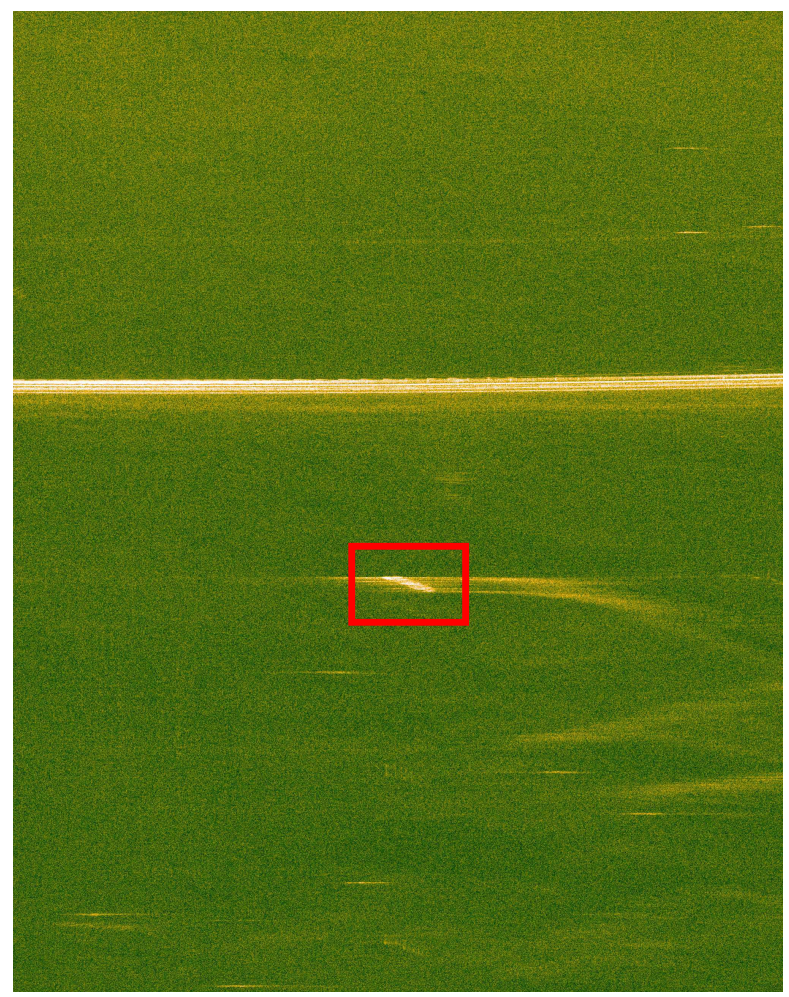

(a)

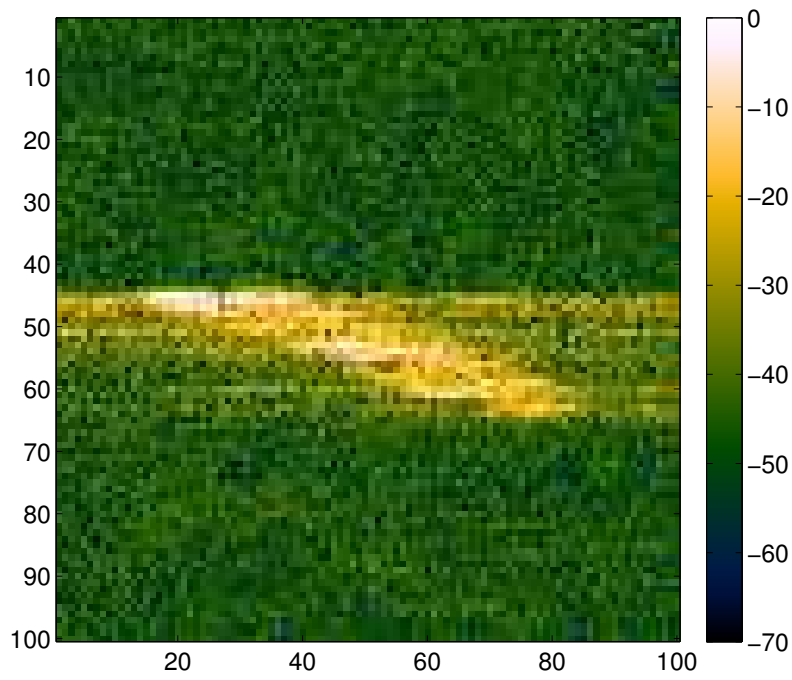

(b)

Figure 5.2: (a) A larger view of the moving target scene 1 where a ship is highlighted by a red box. (b) Selected region containing the moving target. 


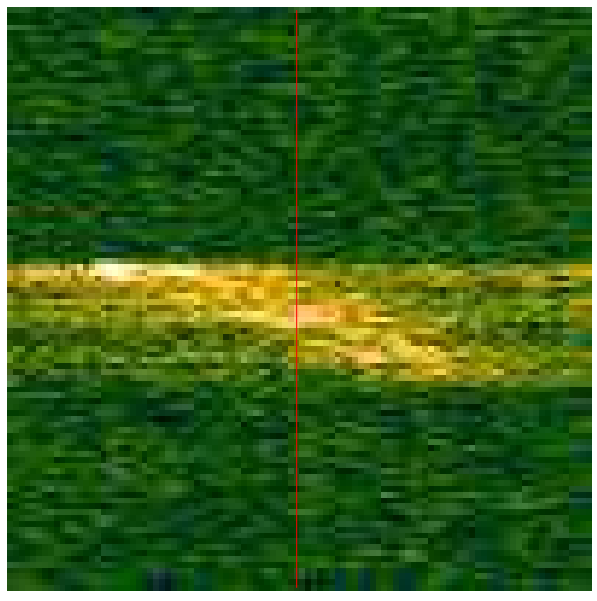

(a)

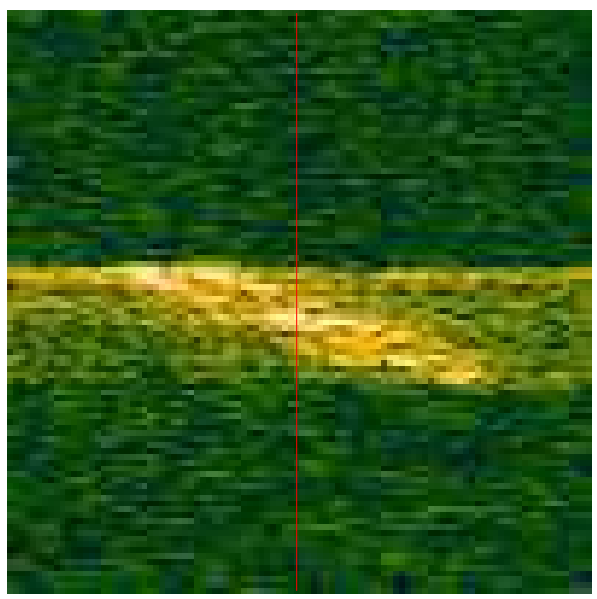

(c)

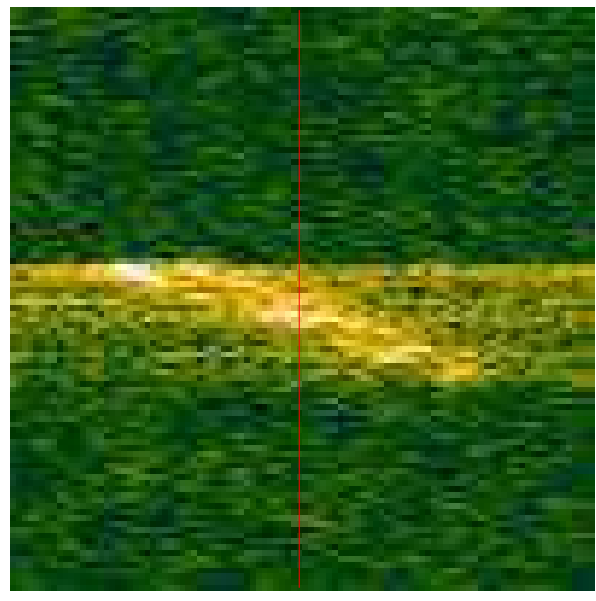

(b)

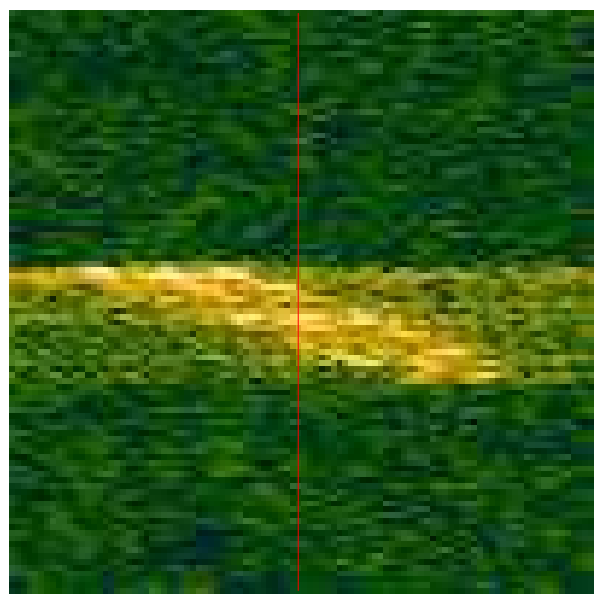

(d)

Figure 5.3: Composite subaperture images of the moving target scene 1. (a) Subaperture image 1. (b) Subaperture image 2. (c) Subaperture image 3. (d) Subaperture image 4 . 


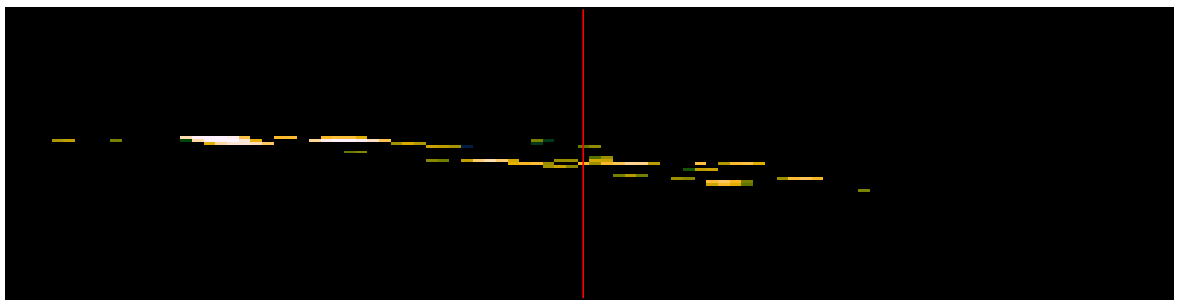

(a)

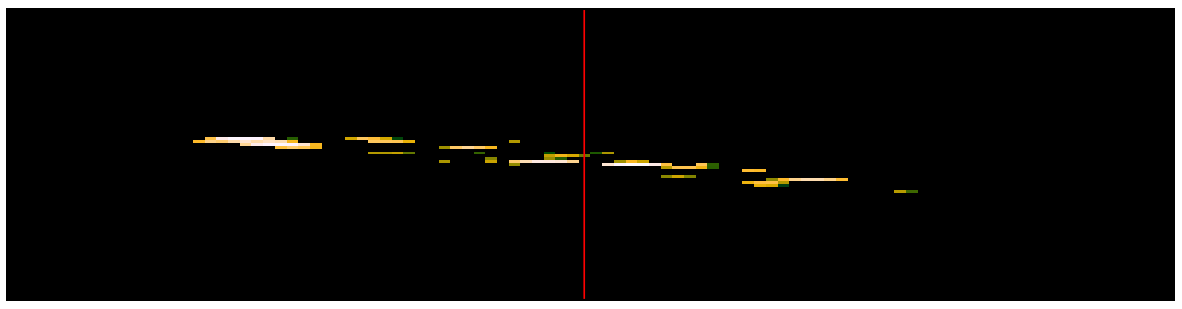

(b)

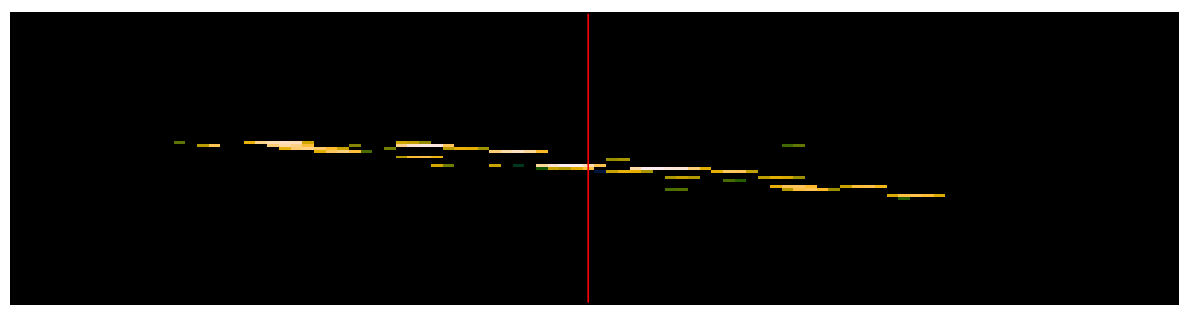

(c)

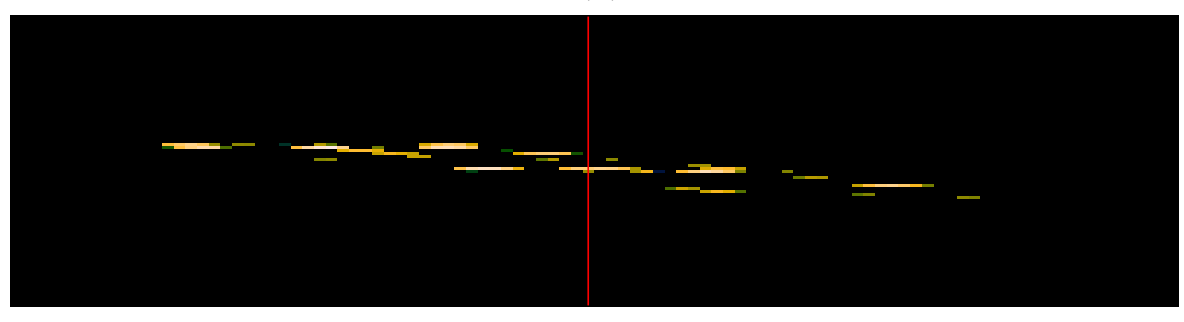

(d)

Figure 5.4: Sparse subaperture images of the moving target scene 1. (a) Subaperture image 1. (b) Subaperture image 2. (c) Subaperture image 3. (d) Subaperture image 4. 


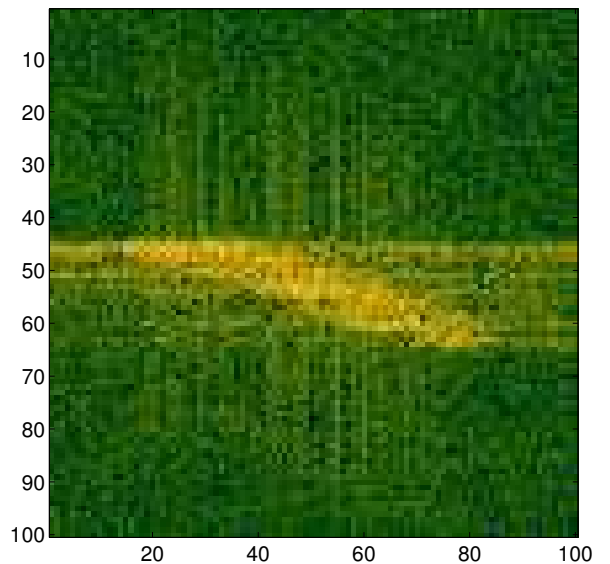

(a)

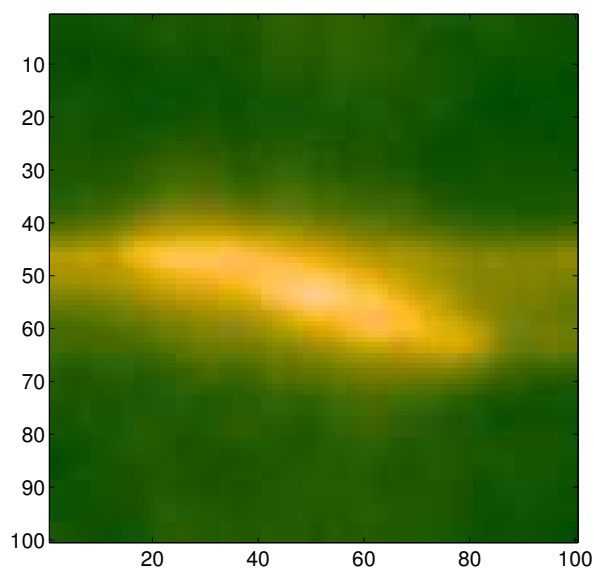

(c)

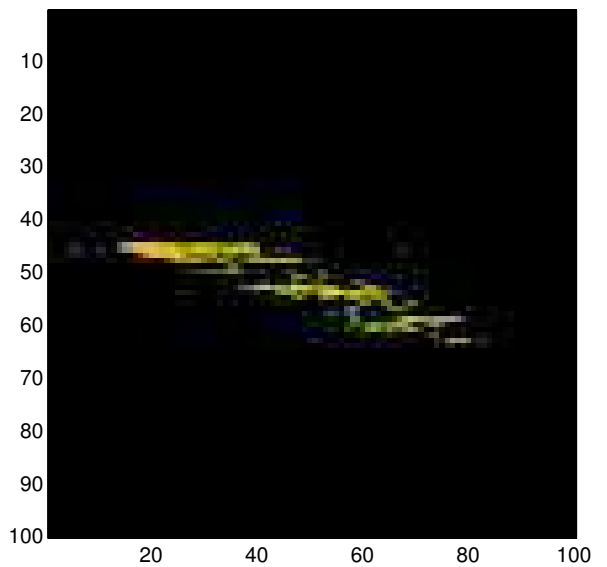

(b)

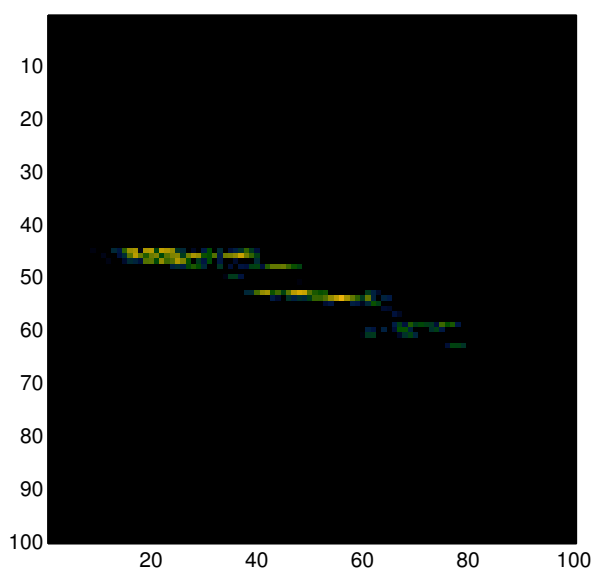

(d)

Figure 5.5: Full-resolution reconstruction results of SLRSD for the moving target scene 1. (a) Full-resolution background SAR image. (b) Full-resolution sparse SAR image. (c) Region-enhanced background SAR image. (d) Sparsity-driven focused SAR image. 


\subsection{Moving Target Scene 2 (region-2)}

As a second part of our EMISAR experiments, we have used region-2 from the overall EMISAR scene shown in Figure 5.1. A magnified view of this area is shown in Figure 5.6(a). There are many small moving targets in this area and we have identified two moving targets with a red box which are lying in a closed vicinity to each other as shown in Figure 5.6(b). Moreover, these targets are mostly moving in the azimuth (horizontal) direction.

A successful decomposition of both moving targets was achieved with our proposed framework. The results are presented in Figure 5.7. The full-resolution background and moving target images are shown in the first row whereas the region-enhanced and SDF focused images are given in the second row of Figure 5.7. Once again, the proposed framework successfully extracts and reconstructs these moving targets despite being smaller in size as compared to the target in scene 1.

Since these targets extend only to a few range bins (vertical direction), we have further analyzed their reflectivity profiles at the various processing stages. In Figure 5.8, some reflectivity plots are shown related to the first moving target (lying in the upper part of Figure 5.7(b)) in scene 2. In particular, these reflectivity profiles correspond to the range bin number 29 of the selected SAR image. In all the plots, we have presented the original composite reflectivity profile along with the other results for comparison purposes. Figure 5.8(a) depicts the low-rank component of the moving target response after SLRSD. The sparse component of the reflectivity profile is given in Figure 5.8(b).

After processing with the SDF, the moving target's reflectivity profile gets focused and two strong peaks are observable in Figure 5.8(c). Since this moving target was extended in the azimuth direction, the two focused points could correspond to its dominant reflecting parts. Similarly, results for the second moving target (range bin $=$ 94) are presented in Figure 5.9 where a single peak is dominant in the SDF focused component. 


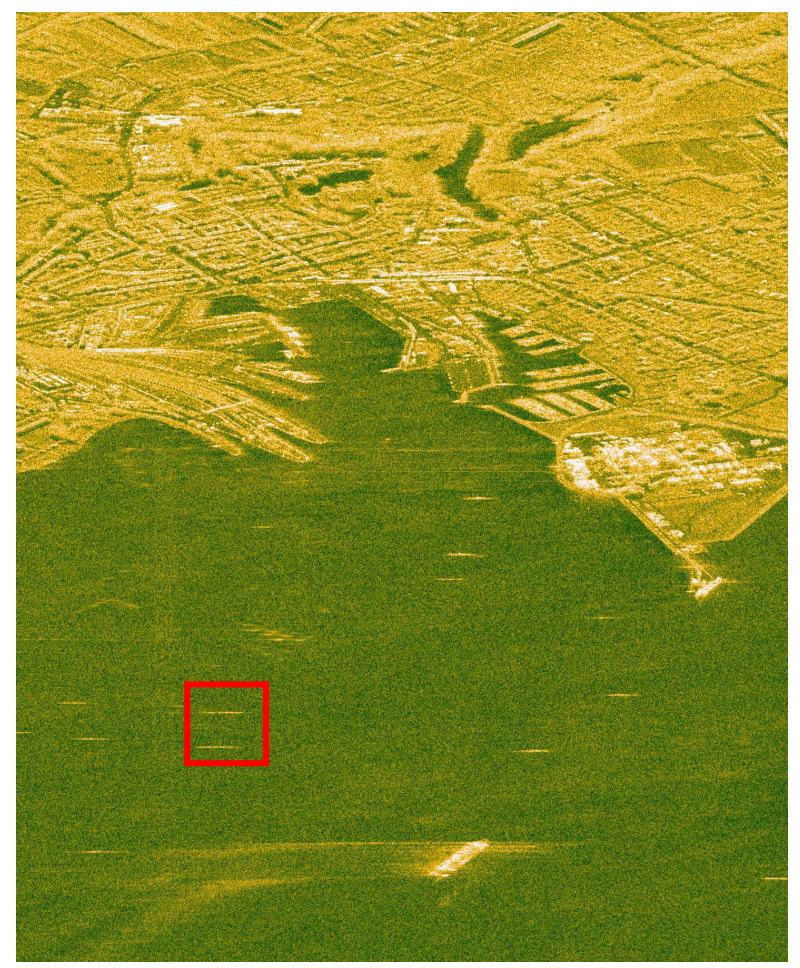

(a)

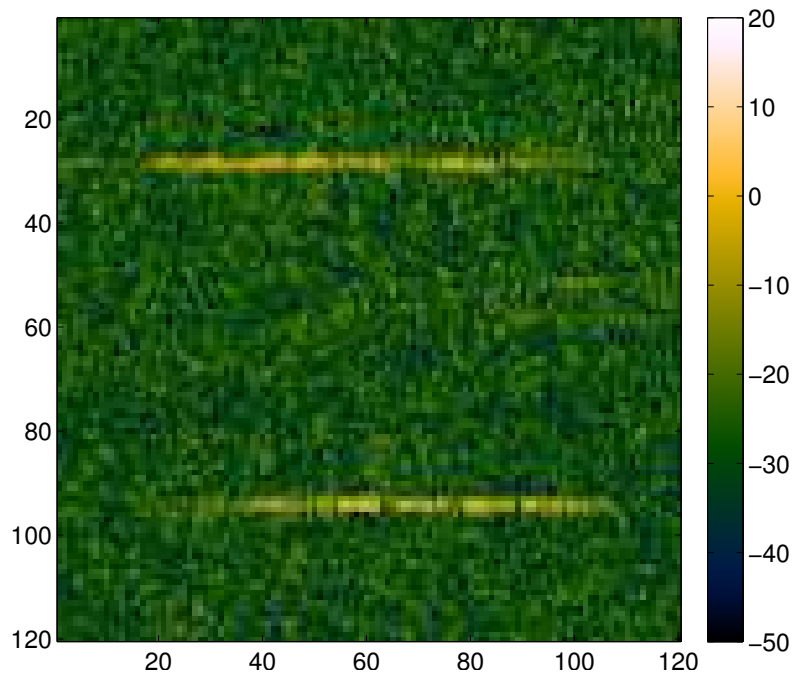

(b)

Figure 5.6: (a) A larger view of the moving target scene 2, where a region containing two moving targets is highlighted by a red box. (b) Selected part of the SAR image containing the moving targets. 


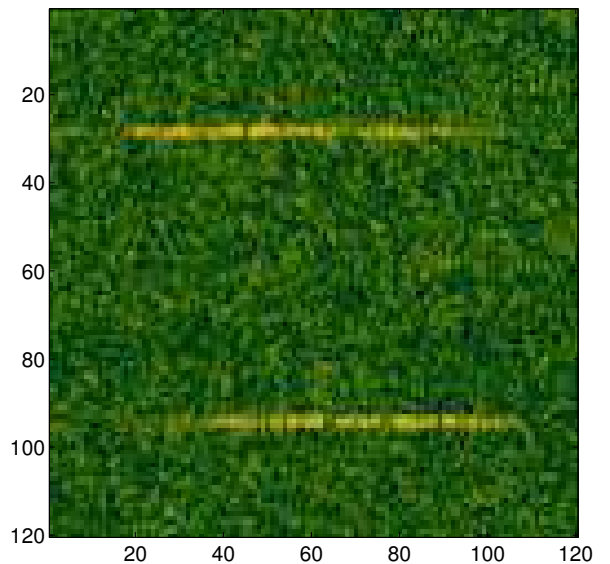

(a)

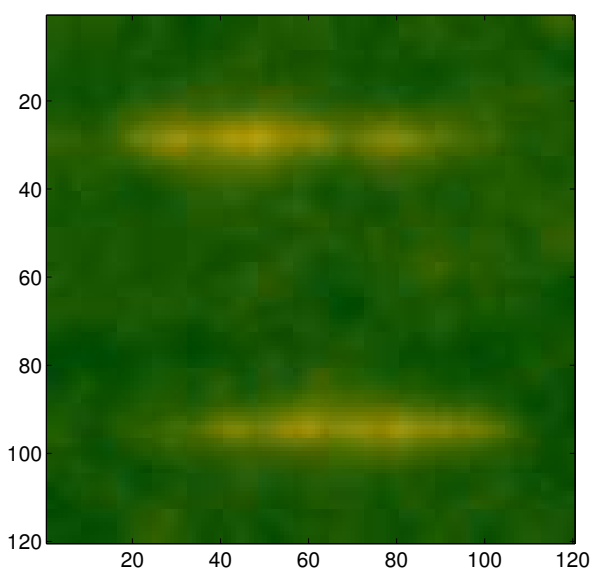

(c)

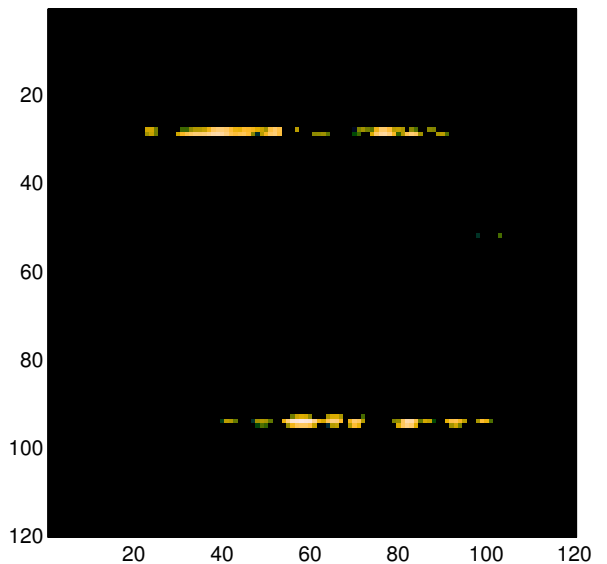

(b)

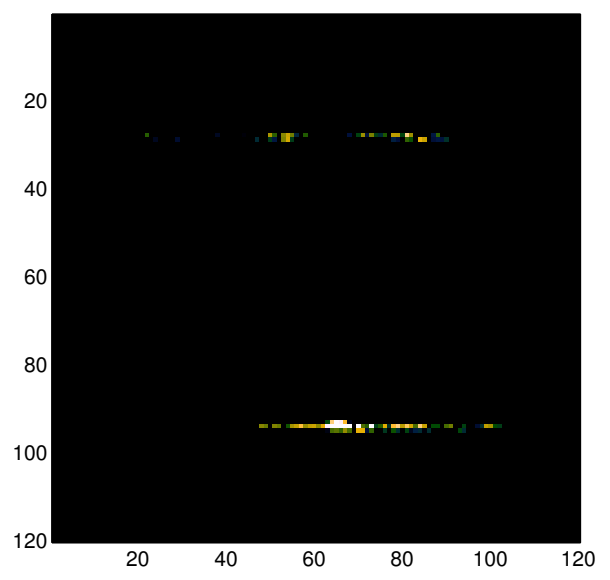

(d)

Figure 5.7: Full-resolution reconstruction results for the moving target scene 2. (a) Full-resolution background SAR image. (b) Full-resolution sparse SAR image. (c) Region-enhanced background SAR image. (d) Sparsity-driven focused SAR image. 


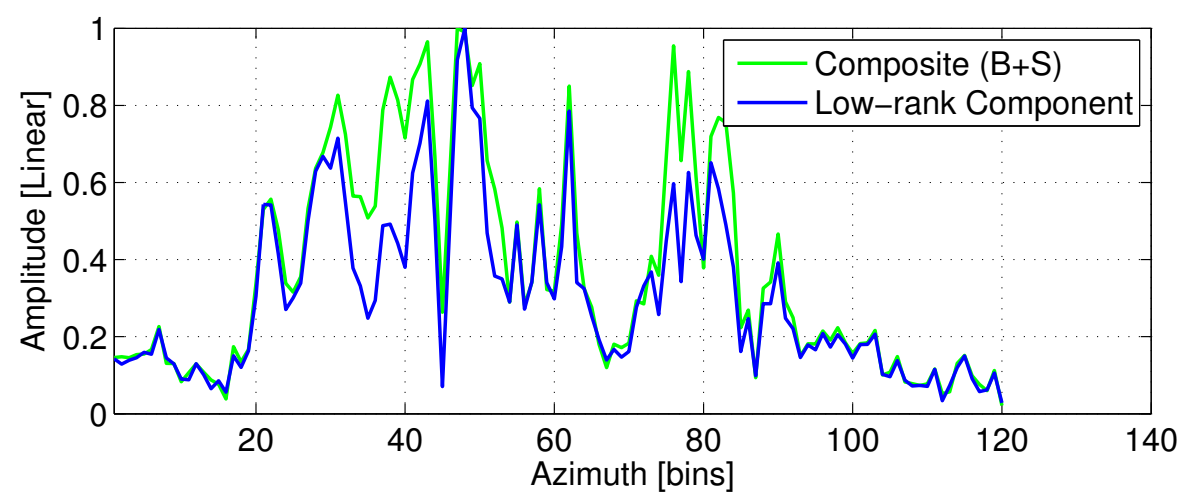

(a)

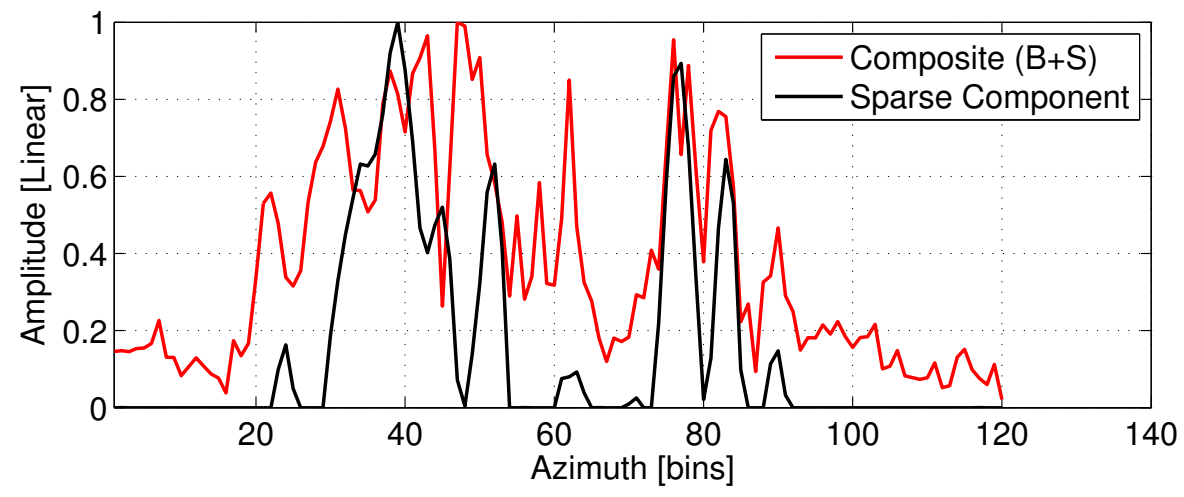

(b)

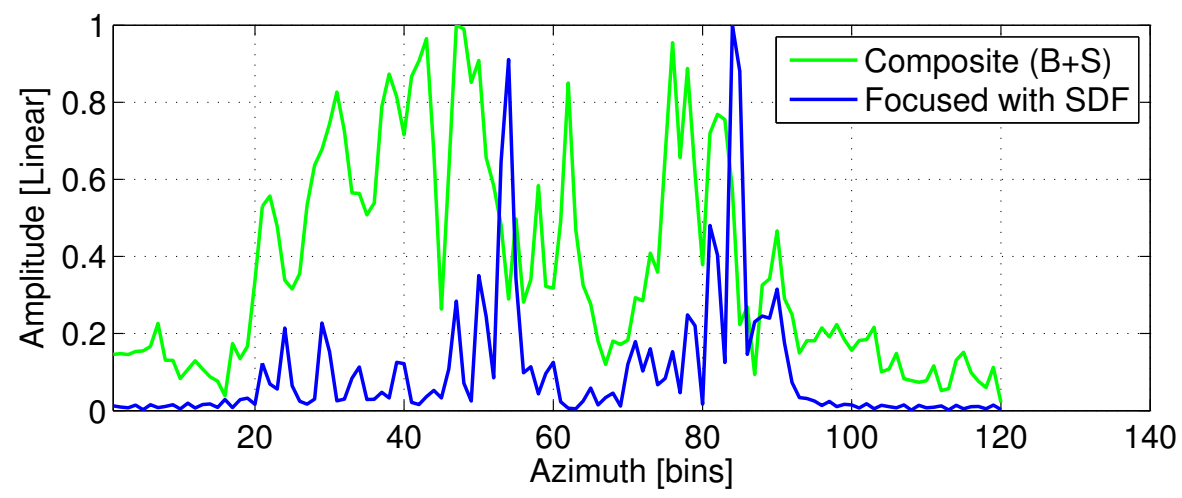

(c)

Figure 5.8: Amplitude reflectivity profiles at various processing stages for the moving target scene 2 (First moving target, range bin $=29)$. (a) Composite signal (B+S, green) and the Low-rank/background component (blue). (b) Sparse component (black). (c) SDF focused component(blue). 


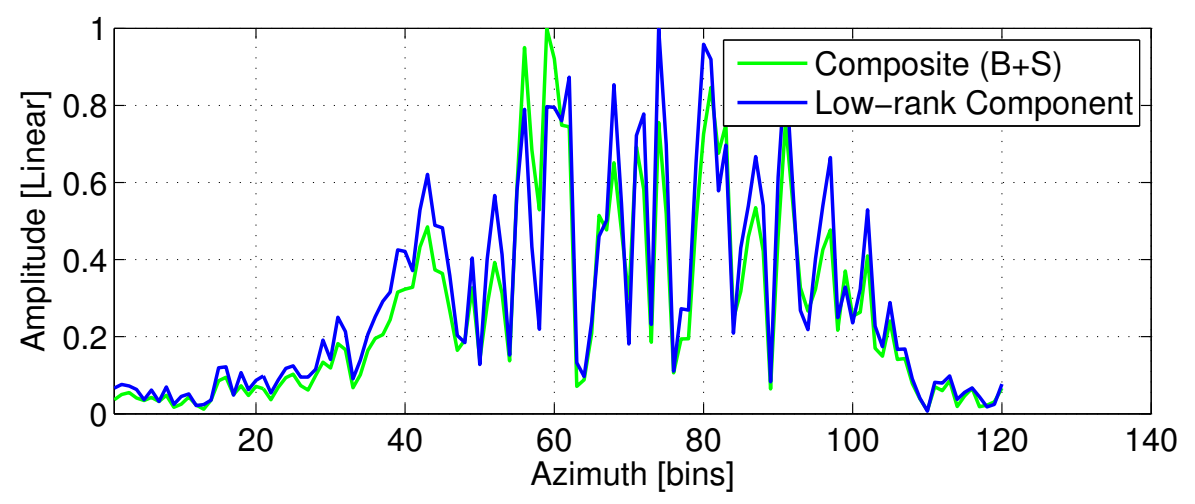

(a)

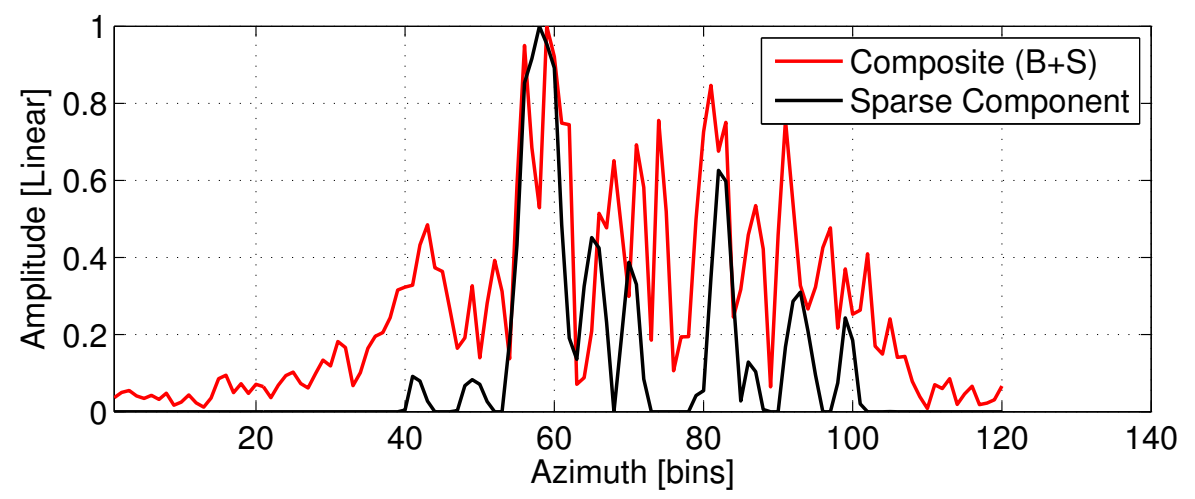

(b)

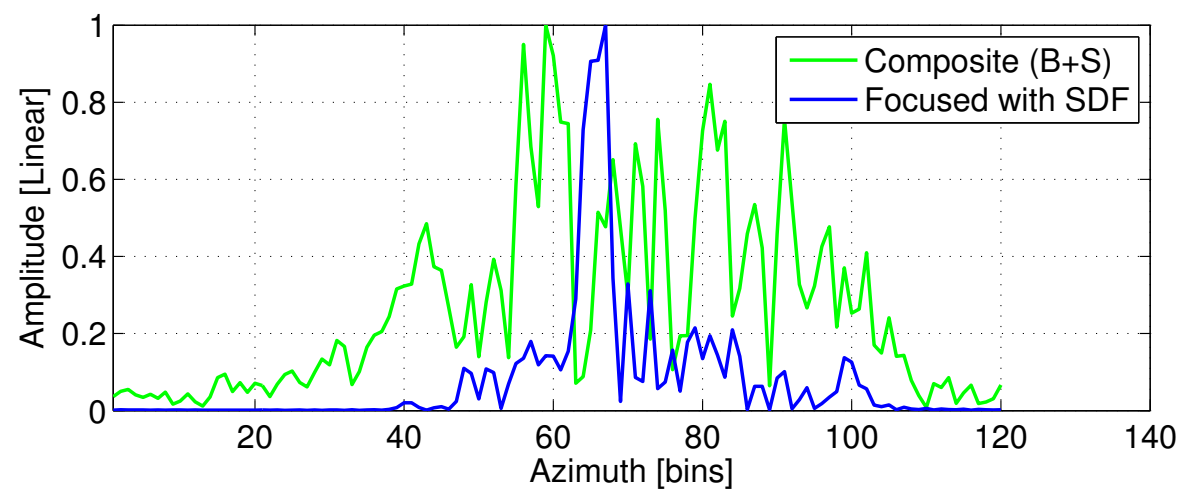

(c)

Figure 5.9: Amplitude reflectivity profiles at various processing stages for the moving target scene 2 (Second moving target, range bin = 94) (a) Composite signal ( $\mathrm{B}+\mathrm{S}$, green) and the Low-rank/background component (blue). (b) Sparse component (black). (c) SDF focused component(blue). 


\subsection{Moving Target Scene 3 (region-2)}

Finally, we have selected another moving target from the region-2 of overall EMISAR scene. It is a large vessel which is heading in a diagonal direction (north-east). The azimuth velocity component of this target is almost opposite to that of the moving target in scene 1 which was heading north-west. The location of this moving target in region-2 is highlighted in Figure 5.10(a) and the selected moving target scene 3 $(240 \times 240)$ is shown in Figure 5.10(b).

The four composite subaperture images are shown in Figure 5.11 which were used for the SLRSD processing. A little movement of the moving target in different subapertures is noticeable which is sufficient for a successful decomposition of the scene.

The conventionally reconstructed full-resolution images of the decomposed background component and the moving target component are shown in Figure 5.12(a) and Figure $5.12(\mathrm{~b})$ respectively.

The region-enhanced reconstruction of the full-resolution background component is given in Figure 5.13(a). Moreover, a point-enhanced reconstruction for the fullresolution moving target image was also carried out. The results are shown in Figure 5.13(b) which is very similar to the full-resolution moving target image except for a small degree of point features improvement. The SDF processed moving target image is shown in Figure 5.14.

\subsection{Discussion}

EMISAR data contains various sizes of targets that are moving with different velocities and headings. There are several conclusions that could be drawn based on our results with this real SAR data. Firstly, our proposed method extracted and reconstructed moving targets of different sizes. Secondly, these moving targets had different azimuth velocities and they were successfully decomposed as the sparse component. Lastly, we were able to detect moving targets even if they were not exactly heading in azimuth direction (e.g., scene 1 and 3). A very small azimuth component of the overall target velocity vector could be sufficient for a successful SLRSD. 


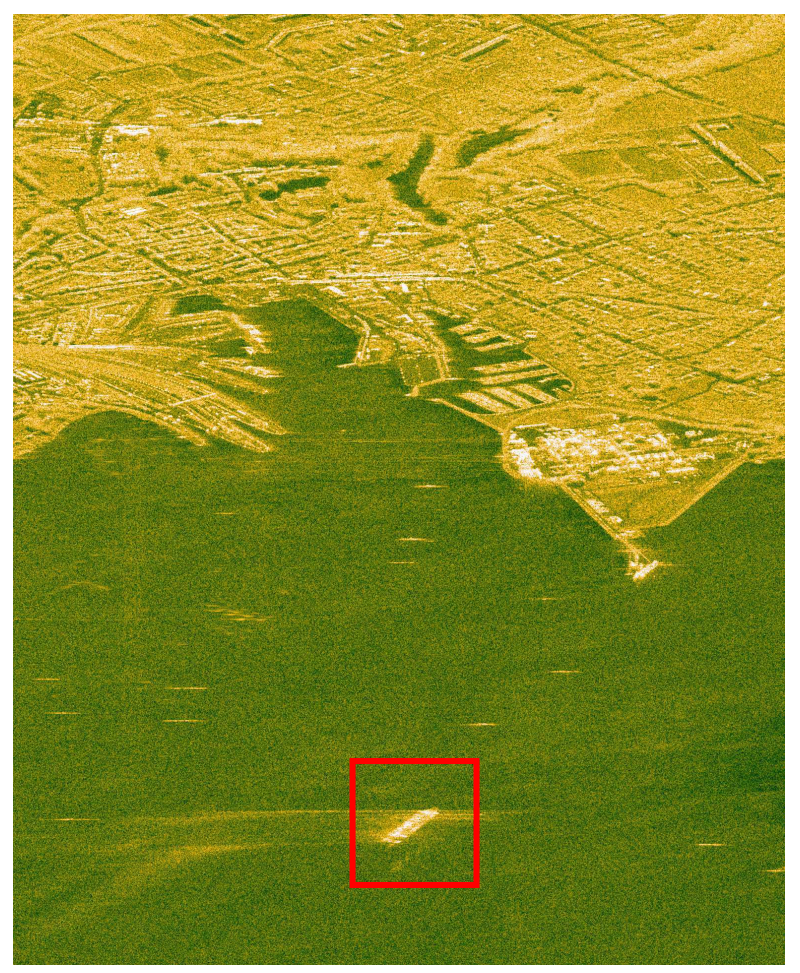

(a)

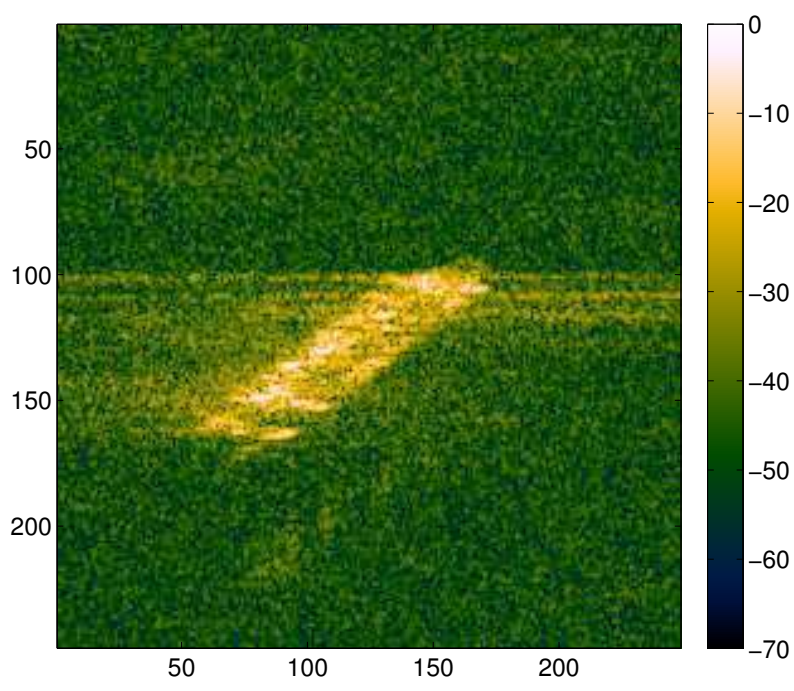

(b)

Figure 5.10: (a) A larger view of the moving target scene 3. A large vessel is highlighted by the red box. (b) Selected part of the SAR image containing the moving target. 


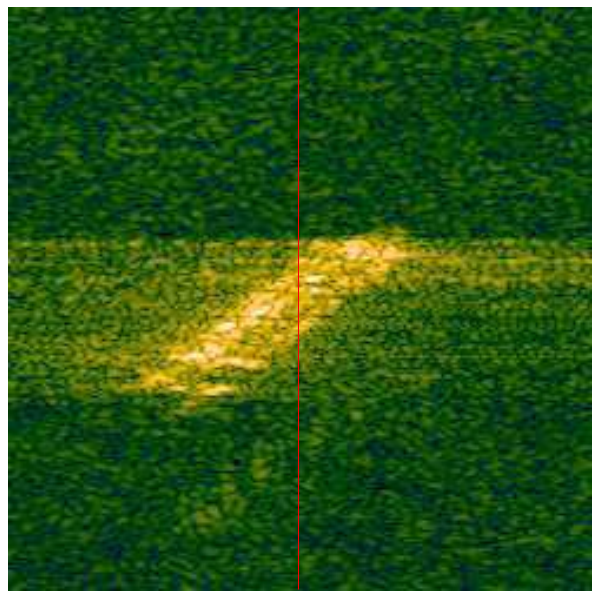

(a)

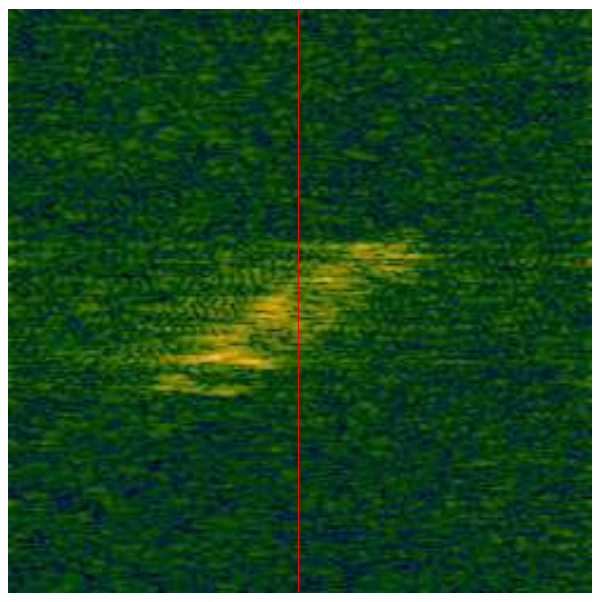

(c)

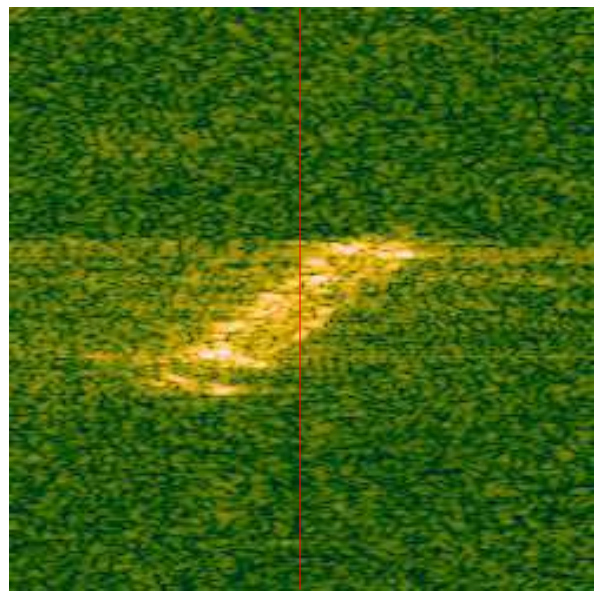

(b)

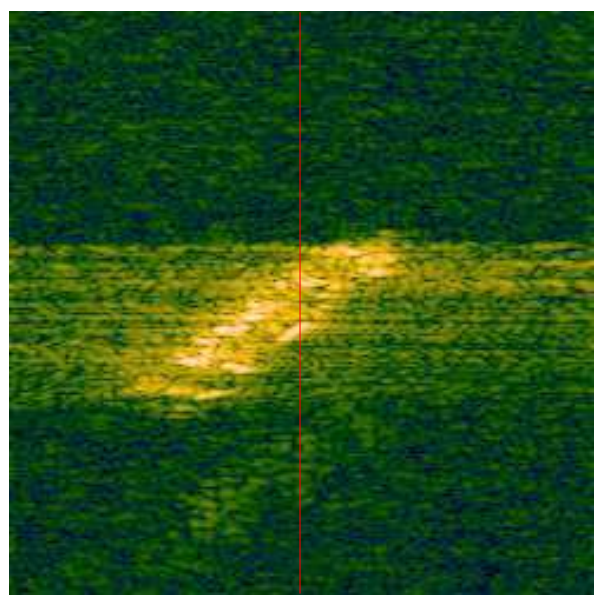

(d)

Figure 5.11: Composite subaperture images of the moving target scene 3. (a) Subaperture image 1. (b) Subaperture image 2. (c) Subaperture image 3. (d) Subaperture image 4 . 


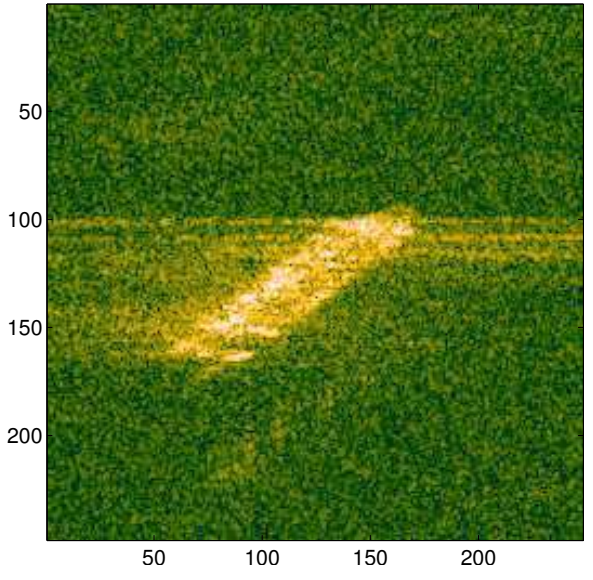

(a)

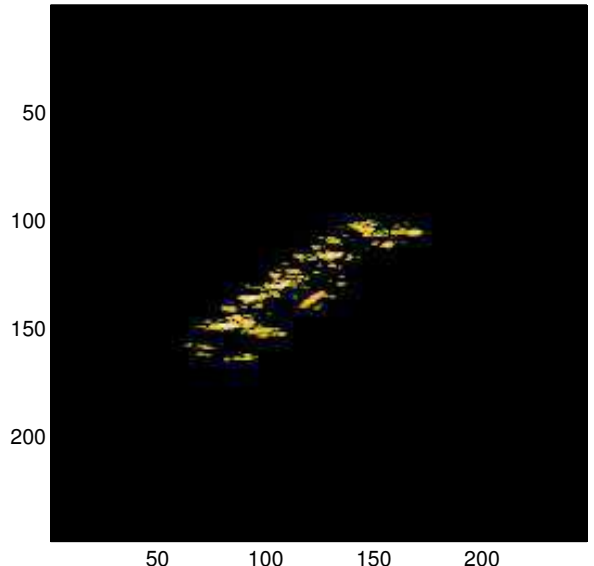

(b)

Figure 5.12: Full-resolution reconstruction results for the moving target scene 3. (a) Full-resolution background image. (b) Full-resolution sparse image which mostly contains the moving target.

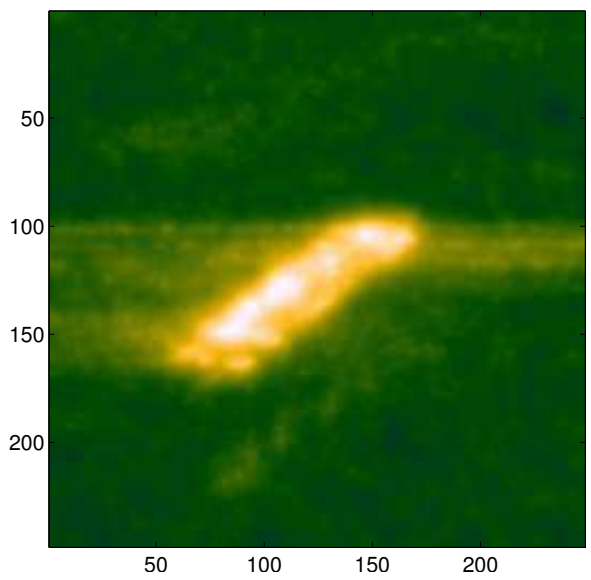

(a)

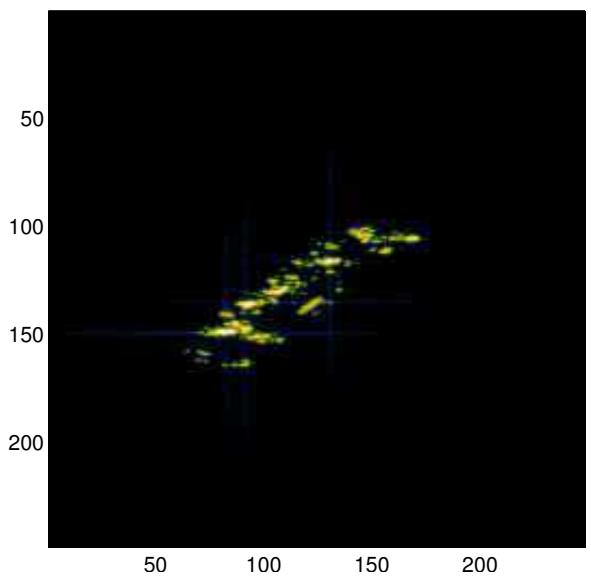

(b)

Figure 5.13: Point and region enhanced reconstructions for the moving target scene 3.

(a) Region-enhanced background image. (b) Point-enhanced moving target image. 


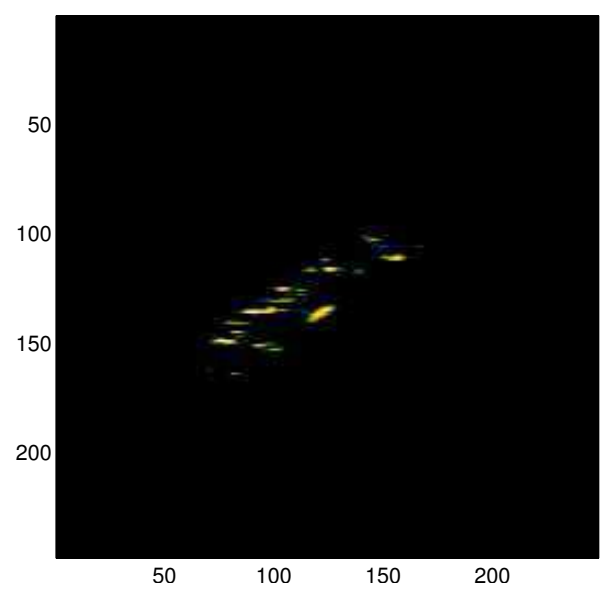

Figure 5.14: Sparsity-driven focusing results for the moving target scene 3. 


\section{Chapter 6}

\section{Conclusions and Future Work}

\subsection{Conclusions}

This thesis contributes to the problem of moving target imaging with SAR by proposing a new approach. In a typical SAR scene, moving targets produce phase errors as compared to the static points. This demands some extra processing for their proper imaging and motion parameter estimation. Nevertheless, the fundamental processing step is the detection of moving targets from a largely static background. Conventional filtering-based techniques for single antenna SAR systems have severe performance limitations. Although multi-antenna SAR systems can produce more robust phase-based detection, generally their cost, size, and complexity are prohibitive.

In this thesis, a moving target imaging approach for SAR has been proposed that employs a subaperture based low-rank and sparse decomposition for the extraction of moving targets from the stationary background. Moving targets in a SAR scene change their position in the various subapertures. This dynamic behavior across subapertures leads to their decomposition as the sparse component. A salient feature of this approach is its capability to reconstruct full-resolution moving target images after the LRSD decomposition. Moreover, this approach extends the applicability of sparsity-driven moving target imaging to very low SCR scenarios with about a $10 \mathrm{~dB}$ SCR performance improvement. Additionally, this framework performs very well for the slow moving targets. In our experiments on small scenes, a fewer number of subapertures were preferred for better performance with an added advantage of less memory requirement 
and faster processing time. Experimental results with synthetic and semi-synthetic SAR data demonstrate the potential of our approach. Applicability and performance with real SAR scenes containing actual moving targets is affirmed by experiments with EMISAR data.

\subsection{Future Work}

Several possibilities of future work originate from this research.

- Firstly, the real SAR imagery has some anisotropic components especially in the urban environments where this is more pronounced. A resolution cell in the SAR image is said to have anisotropic behavior if its reflectivity varies with the exposure angle. This behavior can lead to background amplitude variations in the different subaperture images causing the leakage of some background components into the sparse part. Modeling of the anisotropy and its inclusion into the moving target imaging framework is a potential future work.

- We have tuned the regularization parameters $\lambda_{b}, \lambda_{s}$, and $\lambda_{p}$ using a trial and error approach. However, in future these parameters could be calculated automatically by mathematical modeling based on signal to clutter ratio, speckle level or some measure of anisotropy in the scene. This may lead to further performance improvement.

- We have observed that the moving target's reflectivity profiles contains low-rank components as well which are included in the background image as a residual. Modeling of this effect and analysis of its dependence on target velocity and number of subapertures could be carried out in future.

- Inclusion of a motion parameter estimation technique into this proposed framework could be a useful extension. Since most of the phase history information of moving targets is captured by the sparse component, applicability of existing motion parameter estimation approaches could possibly be extended to low SCR scenarios by combining them with the proposed SLRSD framework. Moreover, 
the response of moving targets in the subaperture images and decomposed image components after SLRSD could be useful to determine the azimuth velocity of the target.

- Application of our proposed moving target imaging framework to very large SAR scenes could be an interesting work. Moreover, considering the availability of low-cost parallel processing hardware, reformulation of our method for a parallel implementation could be a useful direction to work on. In this regard, there could be two approaches: 1) A windowing based processing where a very large SAR scene is partitioned into a number of small images each of which is processed by a separate processor in parallel, and 2) A region of interest based approach which processes only selected areas of the scene with our proposed framework where the moving targets are more probable to be found. Examples of such regions include train tracks, highways, and sea routes. 


\section{Bibliography}

[1] M. T. Crockett, "Target motion estimation techniques for single-channel SAR," Master Thesis, Brigham Young University, 2014.

[2] I. G. Cumming and F. H. Wong, "Digital processing of synthetic aperture radar data," Artech House, vol. 1, no. 2, p. 3, 2005.

[3] R. K. Raney, "Synthetic aperture imaging radar and moving targets," IEEE Transactions on Aerospace and Electronic Systems, no. 3, pp. 499-505, 1971.

[4] J. K. Jao, "Theory of synthetic aperture radar imaging of a moving target," IEEE Transactions on Geoscience and Remote Sensing, vol. 39, no. 9, pp. 1984-1992, 2001.

[5] M. Soumekh, Synthetic aperture radar signal processing. New York: Wiley, 1999, vol. 7 .

[6] G. Li, X.-G. Xia, J. Xu, and Y.-N. Peng, "A velocity estimation algorithm of moving targets using single antenna SAR," IEEE Transactions on Aerospace and Electronic Systems, vol. 45, no. 3, 2009.

[7] P. R. Kersten, R. W. Jansen, K. Luc, and T. L. Ainsworth, "Motion analysis in SAR images of unfocused objects using time-frequency methods," IEEE Geoscience and Remote Sensing Letters, vol. 4, no. 4, pp. 527-531, 2007.

[8] E. Stockburger and D. Held, "Interferometric moving ground target imaging," in Record of the IEEE 1995 International Radar Conference. IEEE, 1995, pp. 438443. 
[9] F. Qin, X. Zhang, and M. Dong, "A method of hybrid ATI and DPCA technique to detect moving target," in CIE'06, International Conference on Radar, 2006. IEEE, 2006, pp. 1-4.

[10] J. H. Ender, "Space-time processing for multichannel synthetic aperture radar," Electronics \&3 Communication Engineering Journal, vol. 11, no. 1, pp. 29-38, 1999.

[11] C. He, L. Liu, L. Xu, M. Liu, and M. Liao, "Learning based compressed sensing for SAR image super-resolution," IEEE Journal of Selected Topics in Applied Earth Observations and Remote Sensing, vol. 5, no. 4, pp. 1272-1281, 2012.

[12] M. A. Herman and T. Strohmer, "High-resolution radar via compressed sensing," IEEE Transactions on Signal Processing, vol. 57, no. 6, pp. 2275-2284, 2009.

[13] M. Çetin and W. C. Karl, "Feature-enhanced synthetic aperture radar image formation based on nonquadratic regularization," IEEE Transactions on Image Processing, vol. 10, no. 4, pp. 623-631, 2001.

[14] S. Samadi, M. Çetin, and M. A. Masnadi-Shirazi, "Sparse representation-based synthetic aperture radar imaging," IET Radar, Sonar $\mathcal{E}$ Navigation, vol. 5, no. 2, pp. 182-193, 2011.

[15] M. Cetin, I. Stojanovic, O. Onhon, K. Varshney, S. Samadi, W. C. Karl, and A. S. Willsky, "Sparsity-driven synthetic aperture radar imaging: Reconstruction, autofocusing, moving targets, and compressed sensing," IEEE Signal Processing Magazine, vol. 31, no. 4, pp. 27-40, 2014.

[16] L. C. Potter, E. Ertin, J. T. Parker, and M. Cetin, "Sparsity and compressed sensing in radar imaging," Proceedings of the IEEE, vol. 98, no. 6, pp. 1006-1020, 2010.

[17] N. Ö. Önhon and M. Cetin, "SAR moving target imaging in a sparsity-driven framework," in SPIE Optical Engineering + Applications. International Society for Optics and Photonics, 2011, pp. 813806-813806. 
[18] N. Ö. Önhon and M. Çetin, "SAR moving object imaging using sparsity imposing priors," EURASIP Journal on Advances in Signal Processing, vol. 2017, no. 1, p. 10, 2017.

[19] R. Otazo, E. Candès, and D. K. Sodickson, "Low-rank plus sparse matrix decomposition for accelerated dynamic MRI with separation of background and dynamic components," Magnetic Resonance in Medicine, vol. 73, no. 3, pp. 1125-1136, 2015.

[20] S. G. Lingala, Y. Hu, E. DiBella, and M. Jacob, "Accelerated dynamic mri exploiting sparsity and low-rank structure: kt slr," IEEE Transactions on Medical Imaging, vol. 30, no. 5, pp. 1042-1054, 2011.

[21] X. Zhou, C. Yang, and W. Yu, "Moving object detection by detecting contiguous outliers in the low-rank representation," IEEE Transactions on Pattern Analysis and Machine Intelligence, vol. 35, no. 3, pp. 597-610, 2013.

[22] D. Yang, G. Liao, S. Zhu, X. Yang, and X. Zhang, "SAR imaging with undersampled data via matrix completion," IEEE Geoscience and Remote Sensing Letters, vol. 11, no. 9, pp. 1539-1543, Sept 2014.

[23] M. Dao, L. H. Nguyen, and T. D. Tran, "Temporal rate up-conversion of synthetic aperture radar via low-rank matrix recovery," in IEEE International Conference on Image Processing, ICIP 2013, Melbourne, Australia, September 15-18, 2013. IEEE, 2013, pp. 2358-2362.

[24] L. Borcea, T. Callaghan, and G. Papanicolaou, "Synthetic aperture radar imaging and motion estimation via robust principal component analysis," SIAM Journal on Imaging Sciences, vol. 6, no. 3, pp. 1445-1476, 2013.

[25] K.-Y. Ni and S. Rao, "SAR moving target imaging using sparse and low-rank decomposition," in SPIE Defense+ Security. International Society for Optics and Photonics, 2014, pp. 90 771D-90 771D. 
[26] E. Mason and B. Yazici, "Moving target imaging using sparse and low-rank structure," in SPIE Defense+ Security. International Society for Optics and Photonics, 2016, pp. 98 430D-98 430D.

[27] F. Biondi, "Low rank plus sparse decomposition of synthetic aperture radar data for maritime surveillance," in 4th International Workshop on Compressed Sensing Theory and its Applications to Radar, Sonar and Remote Sensing (CoSeRa), 2016. IEEE, 2016, pp. 75-79.

[28] A. Soğanlı and M. Cetin, "Low-rank sparse matrix decomposition for sparsitydriven SAR image reconstruction," in 3rd International Workshop on Compressed Sensing Theory and its Applications to Radar, Sonar and Remote Sensing (CoSeRa), 2015. IEEE, 2015, pp. 239-243.

[29] M. Yasin, M. Çetin, and A. S. Khwaja, "SAR imaging of moving targets by subaperture based low-rank and sparse decomposition," in 25th Signal Processing and Communications Applications Conference (SIU), 2017. IEEE, 2017, pp. 1-4.

[30] M. I. Skolnik, "Radar handbook," 1970.

[31] C. E. Cook, "Pulse compression-key to more efficient radar transmission," Proceedings of the IRE, vol. 48, no. 3, pp. 310-316, 1960.

[32] D. C. Munson, Jr., J. D. O'Brien, and W. K. Jenkins, "A tomographic formulation of spotlight-mode synthetic aperture radar," Proc. IEEE, vol. PROC-71, pp. 917925, 1983.

[33] J. R. Fienup, "Detecting moving targets in SAR imagery by focusing," IEEE Transactions on Aerospace and Electronic Systems, vol. 37, no. 3, pp. 794-809, 2001.

[34] A. C. Bovik, Handbook of image and video processing. Academic press, 2010.

[35] D. L. Phillips, "A technique for the numerical solution of certain integral equations of the first kind," J. ACM, vol. 9, no. 1, pp. 84-97, Jan. 1962. 
[36] A. N. Tikhonov, "Solution of incorrectly formulated problems and the regularization method," Soviet Math. Dokl., vol. 4, pp. 1035-1038, 1963.

[37] L. I. Rudin, S. Osher, and E. Fatemi, "Nonlinear total variation based noise removal algorithms," Phys. D, vol. 60, no. 1-4, pp. 259-268, Nov. 1992.

[38] J.-L. Starck, M. Elad, and D. Donoho, "Image decomposition via the combination of sparse representations and a variational approach," IEEE Transactions on Image Processing, vol. 14, no. 10, pp. 1570-1582, Oct 2005.

[39] E. J. Candès, X. Li, Y. Ma, and J. Wright, "Robust principal component analysis?" J. ACM, vol. 58, no. 3, pp. 11:1-11:37, Jun. 2011.

[40] T. B. C. Guyon and E. Zahzah, Robust Principal Component Analysis for Background Subtraction: Systematic Evaluation and Comparative Analysis. INTECH, 1995.

[41] E. Candes and B. Recht, "Exact matrix completion via convex optimization," Communications of the ACM, vol. 55, no. 6, pp. 111-119, 2012.

[42] B. Recht, M. Fazel, and P. A. Parrilo, "Guaranteed minimum-rank solutions of linear matrix equations via nuclear norm minimization," SIAM review, vol. 52, no. 3, pp. 471-501, 2010.

[43] V. Chandrasekaran, S. Sanghavi, P. A. Parrilo, and A. S. Willsky, "Rank-sparsity incoherence for matrix decomposition," SIAM Journal on Optimization, vol. 21, no. 2, pp. 572-596, 2011.

[44] T. Zhou and D. Tao, "Godec: Randomized low-rank \& sparse matrix decomposition in noisy case," in International conference on machine learning. Omnipress, 2011.

[45] J. X. Yuan, "Sparse and Low-Rank Matrix Decomposition Via Alternating Direction Methods," Optimization Online, 2009. 
[46] Z. Lin, M. Chen, and Y. Ma, "The augmented Lagrange multiplier method for exact recovery of corrupted low-rank matrices," ArXiv e-prints, Sep. 2010.

[47] A. Moreira, "Real-time synthetic aperture radar (SAR) processing with a new subaperture approach," IEEE Transactions on Geoscience and Remote sensing, vol. 30, no. 4, pp. 714-722, 1992.

[48] A. Moreira, J. Mittermayer, and R. Scheiber, "Extended chirp scaling algorithm for air-and spaceborne SAR data processing in stripmap and ScanSAR imaging modes," IEEE Transactions on Geoscience and Remote Sensing, vol. 34, no. 5, pp. 1123-1136, 1996.

[49] K. Ouchi, "On the multilook images of moving targets by synthetic aperture radars," IEEE Transactions on Antennas and Propagation, vol. 33, no. 8, pp. $823-827,1985$.

[50] A. Moreira, "Improved multilook techniques applied to SAR and ScanSAR imagery," IEEE Transactions on Geoscience and Remote Sensing, vol. 29, no. 4, pp. 529-534, 1991.

[51] R. Tibshirani, "Regression shrinkage and selection via the lasso," Journal of the Royal Statistical Society. Series B (Methodological), pp. 267-288, 1996.

[52] C. Burroughs. (2004) Sandia lab news. [Online]. Available: http://www.sandia. gov/LabNews/LN02-06-04/labnews02-06-04.pdf

[53] Z. Wang, A. C. Bovik, H. R. Sheikh, and E. P. Simoncelli, "Image quality assessment: from error visibility to structural similarity," IEEE Transactions on Image Processing, vol. 13, no. 4, pp. 600-612, 2004.

[54] C. A. Oxborrow. (2014) EMISAR. [Online]. Available: http://www.space.dtu.dk/english/research/research_divisions/microwaves_ and_remote_sensing/sensors/emisar 